\title{
FRONTAL PLANE HUMERAL ELEVATION EFFECTS ON THE PULLOUT STRENGTH OF SUTURE ANCHORS USED IN ROTATOR CUFF REPAIR
}

\author{
A Thesis \\ Presented to \\ the Faculty of California Polytechnic State University, \\ San Luis Obispo \\ In Partial Fulfillment \\ of the Requirements for the Degree \\ Master of Science in Engineering with Specialization \\ in Biomedical Engineering
}

By

Peter Joshua Larson

June 2010 
(C) 2010

Peter Joshua Larson

ALL RIGHTS RESERVED 


\section{COMMITTEE MEMBERSHIP}

TITLE:

AUTHOR:

DATE SUBMITTED:
Frontal plane humeral elevation effects on the

pullout strength of suture anchors used in rotator cuff repair

Peter Joshua Larson

June 2010

COMMITTEE CHAIR: $\quad$ Lanny Griffin, PhD, Department Chair

COMMITTEE MEMBER: Scott Hazelwood, PhD, Associate Professor

COMMITTEE MEMBER: Jonathan E. Fow, M.D. 


\begin{abstract}
Frontal plane humeral elevation effects on the pullout strength of suture anchors used in rotator cuff repair

By
\end{abstract}

Peter Joshua Larson

The rotator cuff offers the stabilizing forces necessary for fine arm movement. The most common injury to the rotator cuff is the partial or full thickness tear of the supraspinatus tendon, which attaches the humerus to the scapula on the superior side, due to an acute or chronic injury. These injuries account for more than 4.5 million physician visits per year. Depending on the degree of tear, arthroscopic surgery may be needed to reattach the torn tendon to the humeral head. The current preferred method of surgery involves the insertion of a suture anchor into the humeral head that ties the detached tendon using sutures back to bone. Usage of suture anchors promotes healing of the supraspinatus tendon onto the bone re-establishing movement of the arm.

Although they provide a suitable healing interface, suture anchors are prone to various types of failure such as anchor eyelet breakage, suture failure, and anchor pullout. These failure modes necessitate intervention to reattach the tendon thus prolonging the healing period. Identifying and minimizing these failure modes will ensure optimum healing.

There are two goals of this study. Firstly, the paper will validate suture anchor pullout test methodology in comparison with previous studies using polyurethane blocks to ensure robust methods and results. Secondly, this study will investigate the effects of frontal plane humeral elevation on the pullout force of suture anchors. 


\section{ACKNOWLEDGMENTS}

I would like to thank Doctor Lanny Griffin for his technical support, guidance, and advising through the completion of this project.

I would also like to thank Professor Dave Laiho for his extremely kind machining expertise in the creation of the bone holding fixtures.

Lastly, I would like to thank Spiro Atieh for his statistical insights and overall support. 


\section{TABLE OF CONTENTS}

\section{Page}

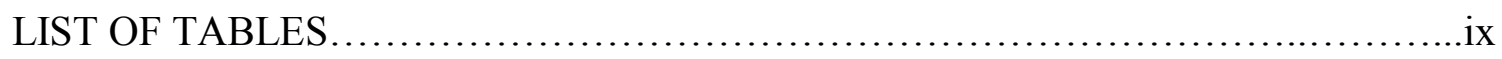

LIST OF FIGURES.......................................................

\section{CHAPTER}

I. CHAPTER 1 INTRODUCTION .........................................

1.1 The Rotator Cuff.............................................

1.2 The Humeral Bone..........................................

1.3 Frontal Elevation of the Humerus...................................

1.4 Rotator Cuff Injury............................................

1.4.1 Rotator Cuff Tear Symptoms.........................4

1.4.2 Diagnosis of a Torn Rotator Cuff........................5

1.4.3 Treatment Options...................................

1.4.4 Tendon Repair Pathway............................. 8

1.5 Types of Suture Anchors.....................................8

1.6 Failure Modes of the Suture Anchor Technique.................... 9

1.6.1 Factors Affecting Pullout Force - Bone.................9

1.6.2 Factors Affecting Pullout Force - Suture Anchors.........10

1.6.3 Factors Affecting Pullout Force - Suture Anchor

Placement..........................................10

II. CHAPTER 2 OBJECTIVES ......................................... 13

III. CHAPTER 3 METHODS AND MATERIALS ............................ 14 
3.1 Suture Anchor Pullout from Polyurethane Blocks Test Setup.

3.2 Humeral Frontal Plane Elevation Angle Effects on Pullout Forces

Test Setup................................................16

3.3 Statistical Methods..............................................20

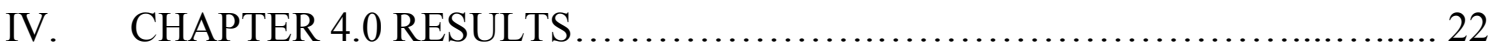

4.1 Pullout Strength of Suture Anchors Implanted into Polyurethane Blocks.

4.2 Humeral Frontal Elevation Angle and Suture Anchor Effects on Pullout Forces..................................................26

V. CHAPTER 5.0 DISCUSSION ......................................... 32

5. 1 Effects of Elevation Angle and Suture Anchor Choice on Pullout

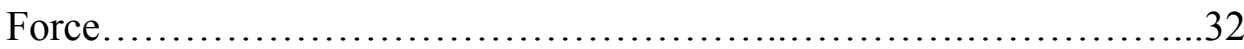

5.2 Pullout Force Test Methodology and Effects of Polyurethane Block Density.....................................................33

5.3 Limitations and Strengths of the Study...........................34

VI. CHAPTER 6.0 CONCLUSIONS/RECOMMENDATIONS FOR FUTURE WORK ........................................................... 36

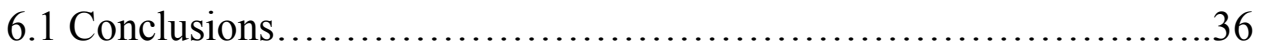

6.2 Recommendations for Future Work...........................36

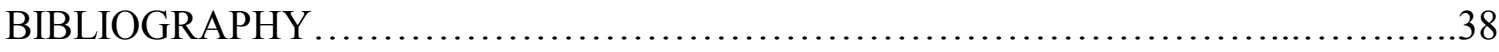

APPENDICES

A. APPENDIX A: DOE Randomized Run Order......................44

B. APPENDIX B: Graphical Summaries of Pullout Tests.................46 
C. APPENDIX C: Derivation of Parametric Estimate Equations............76

D. APPENDIX D: Additional JMP Data Analysis.........................78 


\section{LIST OF TABLES}

Table

Page

Table 3-1: Suture anchors used in the pullout tests from this study as well as the study by Nien. 15

Table 3-2: Density comparison of SAWBONES replica bone and actual humeral

bone......

Table 4-1: Comparison of the peak pullout forces of a $6.5 \mathrm{~mm}$ Smith \& Nephew and a $5 \mathrm{~mm}$ Mitek suture anchor implanted into 5 and $10 \mathrm{lb}$ density polyurethane blocks.

Table 4-2: Comparison of suture anchor pullout forces in an 81b density polyurethane block.

Table 4-3: Pullout forces of 3 different suture anchors at 3 different frontal

elevation angles. 


\section{LIST OF FIGURES}

Figure

Figure 1-1: Shoulder joint internal muscles and bones. (23)......................

Figure 1-2: Anatomical features of humeral head.............................................. 2

Figure 1-3: Full thickness tear of the supraspinatus tendon.........................

Figure 1-4: MRI image of a moderately torn supraspinatus tendon. (26)............5

Figure 1-5: Re-attachment of supraspinatus tendon using suture anchors............. 7

Figure 1-6: Screw type (left) and non-screw type (right) suture anchors........... 8

Figure 1-7: Suture anchor features...................................... 10

Figure 1-8: Suture anchor insertion depth Study by Bynum (4).................. 11

Figure 3-1: SAWBONES polyurethane 10lb/ft3 (left) and 5lb/ft3 (right) density

blocks............................................................... 14

Figure 3-2: 6.5mm Smith \& Nephew Twinfix (left) and 5mm Mitek Spiralok (right) suture anchors................................................. 15

Figure 3-3: The uniaxial pullout of suture anchors test setup.................. 16

Figure 3-4: SAWBONES humeri with distal hole for placement onto potting base..17 Figure 3-5: Isocyanate and Polyol solutions (left). Potted humerus subassembly

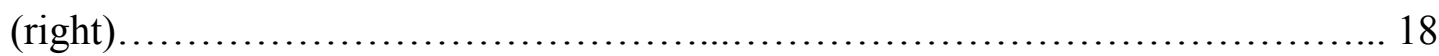

Figure 3-6: 6.5mm Smith \& Nephew Twinfix (left), 5mm Mitek Fastin RC (middle), and 3.5mm Smith \& Nephew Twinfix (right) suture anchors............ 18 Figure 3-7: Potted humeral bone subassembly in bone holding fixture for hypothetical humeral frontal plane angle evaluation (a) $180^{\circ}$, (b) $150^{\circ}$, and (c) $120^{\circ}$ 
Figure 3-8: Experimental setup of potted humeral bone subassembly in bone holding fixture to investigate the effects of humeral bone elevation and suture anchor type on the pullout strength

Figure 4-1: The whole model test for effects of suture anchor pullout in polyurethane blocks.

Figure 4-2: Significance of the block density effect on suture anchor pullout........ 24 Figure 4-3: Significance of the suture anchor effect on suture anchor pullout........25 Figure 4-4: Significance of the block density and suture anchor interaction effect on suture anchor pullout. 25

Figure 4-5: Linear interpolation of 6.5mm Smith \& Nephew and 5mm Mitek suture anchors to obtain pullout force data in an $81 \mathrm{~b}$ density polyurethane block....26 Figure 4-6: The whole model test for effects of suture anchor pullout at various elevations angles.

Figure 4-7: Contributions of the variables, anchor and angle, on the pullout force from the effects test........................................... 30 Figure 4-8: Significance of the elevation angle as well as anchor type effect on suture anchor pullout 


\section{CHAPTER 1 INTRODUCTION}

\subsection{The Rotator Cuff}

The junction of muscles that connect the humerus and scapula is known as the glenohumeral joint (Fig. 1-1). This joint is the most freely moving joint in the body. It is able to undergo movements such as flexion, extension, abduction, adduction, medial and lateral rotation, and horizontal abduction and adduction. (12) The major muscles of the glenohumeral joint are the deltoid, trapezius, serratus anterior, subclavius, pectoralis minor, sternocleidomastoid, levator scapulae, rhomboid major, and rhomboid minor. Under these muscles lies a collection of four smaller muscles, the supraspinatus, infraspinatus, teres minor, and subscapularis, which comprise the rotator cuff. The rotator cuff provides the stabilizing forces needed for fine arm movements around the glenohumeral joint as well as aids in internal rotation, external rotation, and elevation.

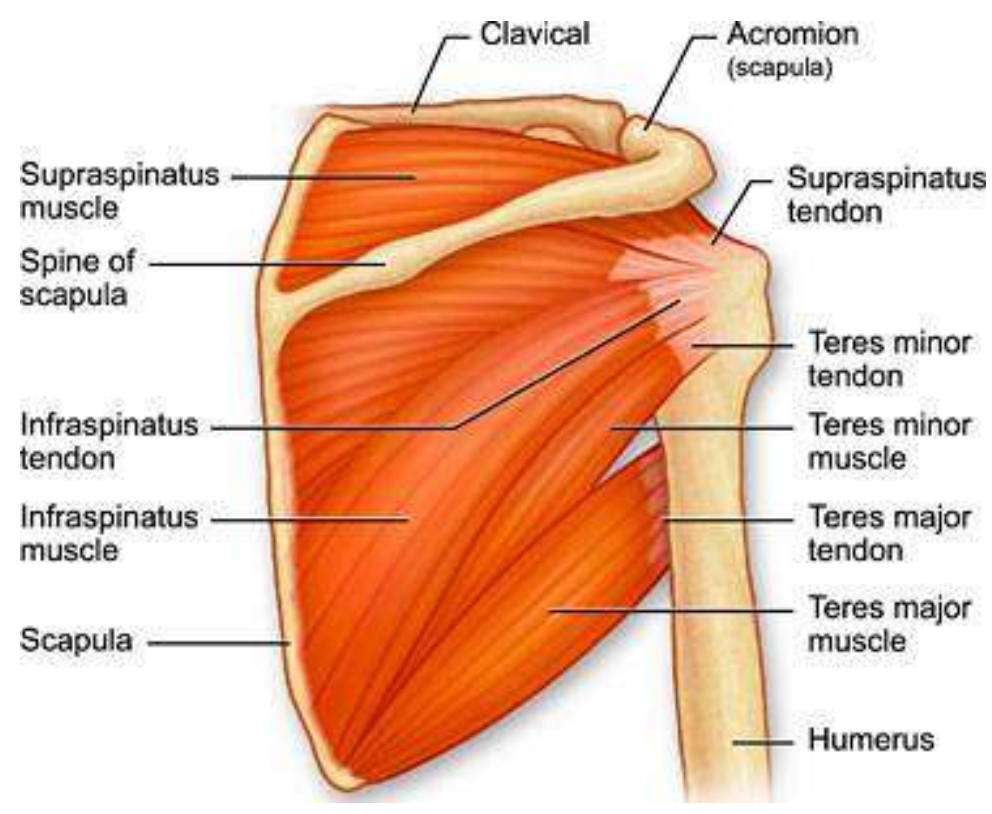

Figure 1-1: Shoulder joint internal muscles and bones. (23) 


\subsection{The Humeral Bone}

The rotator cuff helps to stabilize and control movements of the humerus in the glenoid cavity. This bone is comprised of a head, body, and condyles and it the largest and longest bone in the upper extremity. Its hemispherical head articulates with the scapula during arm movements. The body is almost cylindrical proximally and flattens distally. The condyles work in conjunction with the radius and ulna for articulation. (6)

On the humeral head are zones referred to as tuberosities, which are protruding surfaces of the bone used for the attachment of tendons (Fig 1-2). The greater tuberosity is lateral to the head and distinguished by three flat impressions, the highest of which is the attachment site of the supraspinatus tendon. The lesser tuberosity is more prominent and allows an impression for the subscapularis tendon. Also on the humeral head is an anatomical neck, which separates the head from the tuberosities. (6)

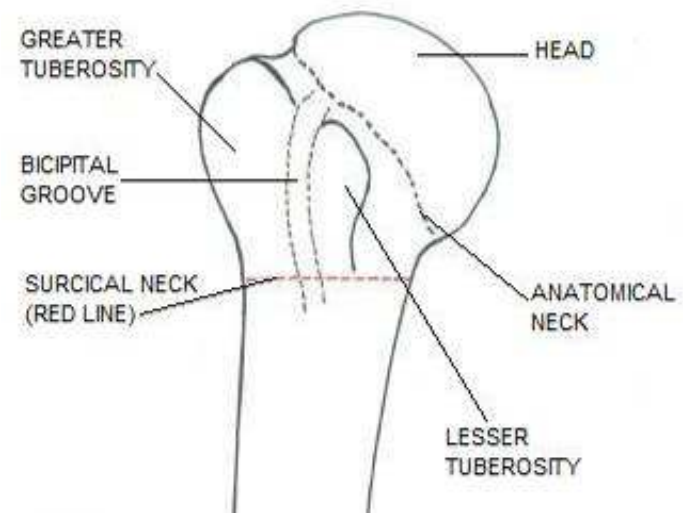

Figure 1-2: Anatomical features of humeral head.

The humeral bone is compromised of two distinct regions, the lower density trabecular bone and the higher density cortical bone. The body of the humerus is primarily made of cortical bone to provide the bone strength. The head of the humerus 
consists of a trabecular tissue that is surrounded by a thin layer of cortical bone for the compressive forces seen during arm movement

\subsection{Frontal Elevation of the Humerus}

Fontal elevation of the shoulder involves humeral and scapular motion. Humeral movement is captured at the glenohumeral joint whereas scapular motion is at the scapulothoracic joint. The movement during the first thirty degrees of shoulder elevation is largely glenohumeral. After thirty degrees, the glenohumeral and scapulothoracic joints move at the same time with a contribution of two to one respectively.

The supraspinatus tendon, which lies directly on top of the humeral head, has been shown to be a significant factor in shoulder elevation. It arises from the supraspinous fossa, runs under the acromion, and attaches to the greater tuberosity. Contraction of this muscle results in abduction of the arm at the shoulder joint. During the first thirty degrees of abduction it is the primary mover, after that the deltoid starts to be the major contributor to elevation. The majority of the elevation force of the supraspinatus comes from the anterior portion of the tendon due to the thick fibrous frame. Studies have found that the forces through the supraspinatus tendon can reach 156 newtons for lateral elevation.(13)

\subsection{Rotator Cuff Injury}

A rotator cuff injury occurs when one of the four tendons gets acutely or chronically damaged. The most common type of rotator cuff injury is the partial or full thickness tear of the supraspinatus tendon. These injuries account for more than 4.5 million physician visits per year in the United States. Partial or full thickness tears occur 
from a multitude of reasons such as age, repeated episodes of minor trauma, use of steroids, tendon inflammation, hypovascularity, and major injuries (Fig 1-3). The largest contributing factor is the wearing away of the tendon, especially at the undersurface of the anterior aspect of the supraspinatus, due to age. (15)

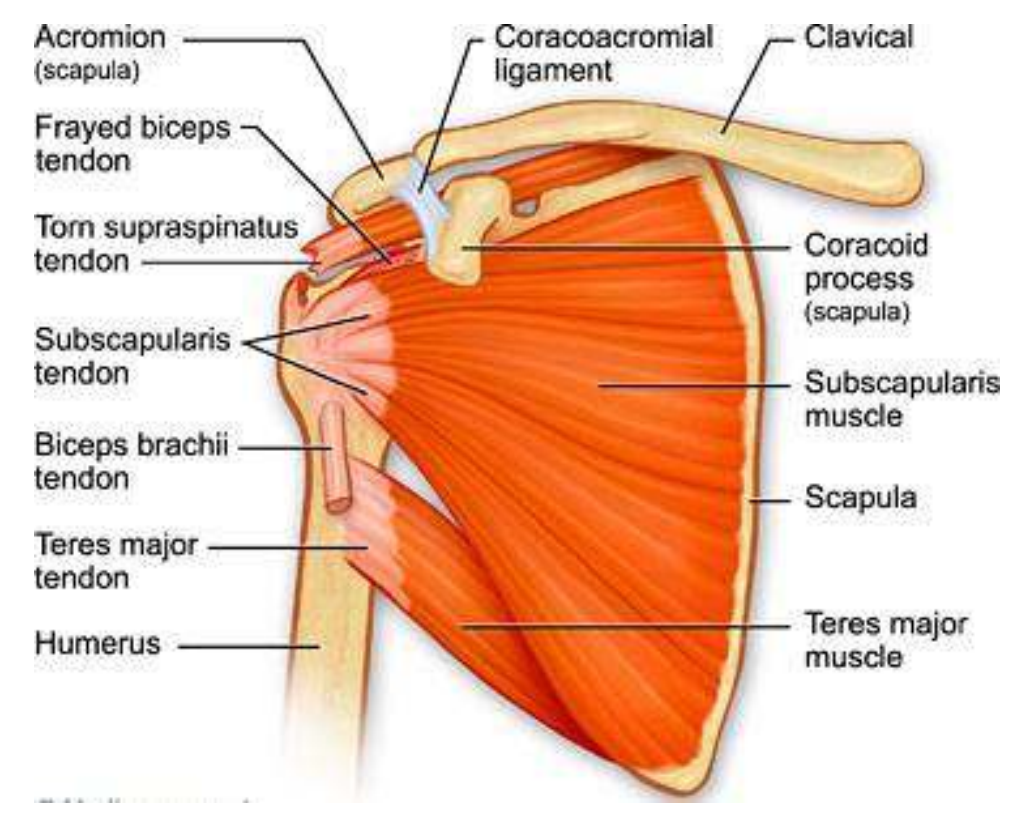

Figure 1-3: Full thickness tear of the supraspinatus tendon.

A fully torn supraspinatus tendon can be classified as either being chronic or acute. A chronic tear is a deterioration of the tendon due to the rubbing of the tendon on the bone over a period of time. An acute tear is from a sudden powerful movement such as a fall or a throw.

\subsubsection{Rotator Cuff Tear Symptoms}

The symptoms of a rotator cuff tear are hard to differentiate from rotator cuff tendonitis or impingement syndrome. Some common symptoms of an acute tear are nighttime awakenings, pain or inability to reach overhead, or radiating pain down the 
shoulder or neck. (15). Chronic tears have a longer onset and gradual increase of weakness but more often than not do not show symptoms.

\subsubsection{Diagnosis of a Torn Rotator Cuff}

High resolution imaging methods, such as MRI and ultrasonography, can be used to evaluate the status of the supraspinatus tendon. Both of these methods have been shown to be almost $90 \%$ accurate in finding full or partial thickness tears. (15) A torn supraspinatus tendon can easily be captured by an MRI (Fig 1-4).

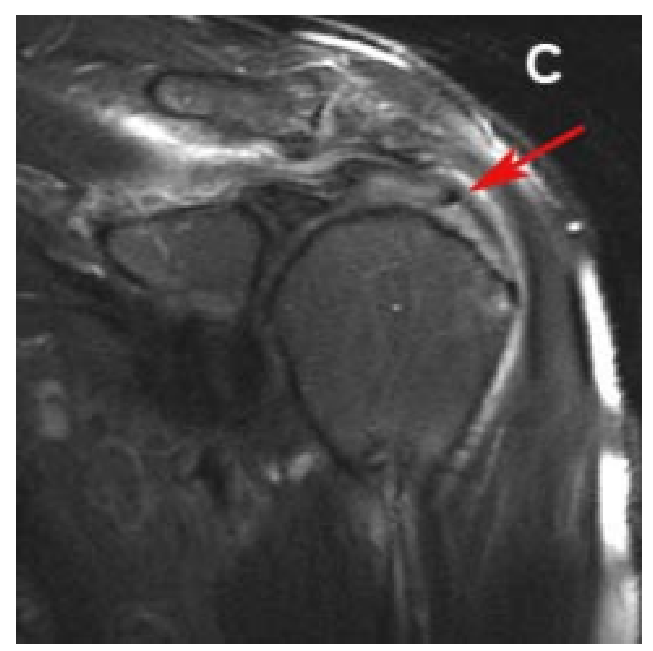

Figure 1-4: MRI image of a moderately torn supraspinatus tendon. (26)

It is also possible to identify defects in the rotator cuff by physical means.

Tremors can be felt on the lateral side of the acromion when the arm is slightly raised to the side. Damage to the supraspinatus can also be identified if there is pain or weakness when the patient isometrically presses up against a resistance with the arm at 90 degrees.

\subsubsection{Treatment Options}

Treatment depends on multiple factors such as the size of the tear, the loss of shoulder function, degree of pain, and age. Partial thickness tears often improve without 
surgery because the remaining attachment of the tendon prevents muscle retraction and atrophy. (15) Physical therapy may be sufficient to treat partial thickness tears by strengthening and fostering the healing of the muscles and tendons. Use of non-steroid anti-inflammatory drugs has also been shown to improve symptoms.

If physical therapy and drugs are not an effective solution, or if the tear is full thickness, surgery is the only option. Treatment of the rotator cuff tear using surgical means is highly successful at alleviating pain and restoring function. Favorable surgery factors are an age less than 60 , short duration of symptoms, no history of smoking, no previous surgery, no muscle atrophy, presence of shoulder stability, good range of should motion, and positive MRI and ultrasound findings. (11)

Currently the most common type of rotator cuff surgery uses suture anchors. These screw like devices are dug into the humeral head exposing an eyelet. Sutures are threaded through this eyelet and connected to the torn tendon. The sutures, usually 2 to 6 , are then pulled taught to bring the tendon to the bone surface and knotted so that tissue healing back onto the humerus may occur. The sutures used for connecting the tendon to the bone cannot replace the strength of a normal tendon, so post operative care must be taken to avoid stressful activities on the healing site. These suture anchors are preferred because of their ease of use, speed of implantation, decreased surgical exposure, and decreased morbidity. The re-attachment of the supraspinatus to the humeral head is commonly performed using suture anchors (Fig 1-5). 


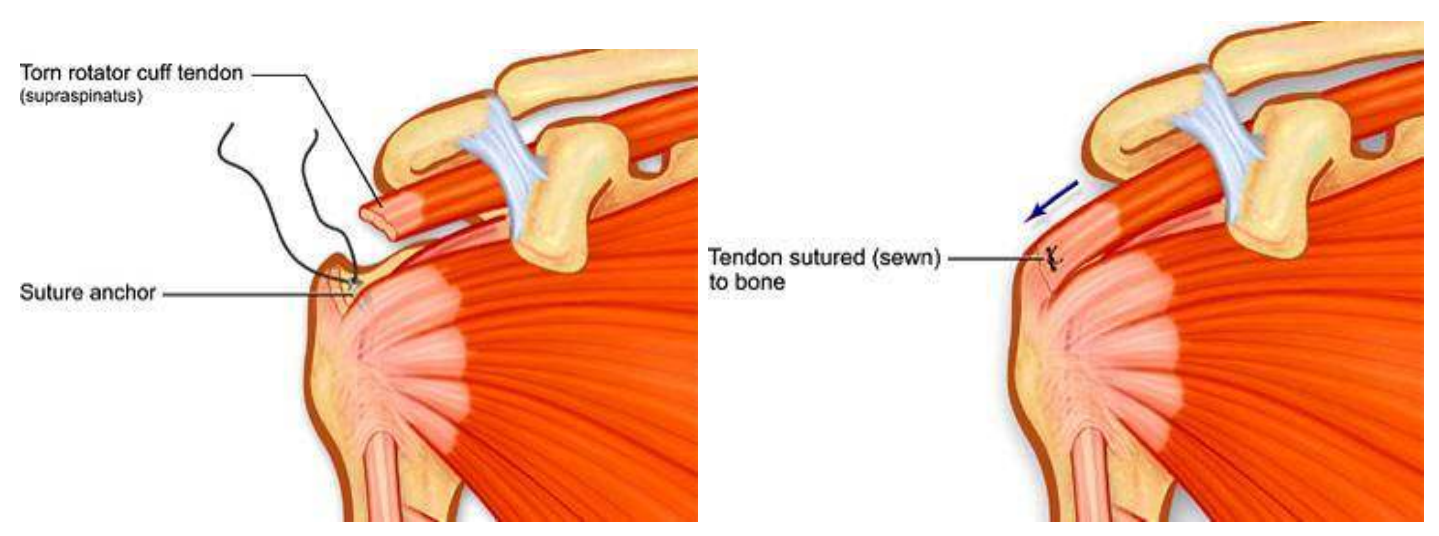

Figure 1-5: Re-attachment of supraspinatus tendon using suture anchors.

Once the tendon is re-attached to the bone, the junction is able to heal. This process depends on four distinct parts: the tendon, the suture, the bone, and the anchor. The sutures connect the tendon to the bone, the bone offers a healing bed for the torn tendon, the anchor provides the attachment site, and the tendon heals onto the bone. To optimize healing doctors must carefully choose the correct anchor and sutures. Some key factors of a suture anchor are configuration, size, shape, and type. For the suture they are material, configuration, and quantity.

Surgery is most often performed on an out patient basis, but overnight stay may be required. Pain is minimized by use of a scalene block, a regional anesthesia. As with all surgical procedure risks could be present such as infection, loss of motion, damage to the deltoid, or nerve damage, but are very rare (15). Physical therapy is very important in the first two months after surgery. Shoulder motion must be regained in a passive mode to help minimize pain, stiffness, and protect the repair. Repaired cuffs must be protected for at least 3 months and the total rehabilitation time is 1 year. 


\subsubsection{Tendon Repair Pathway}

Once damaged, tissue healing goes through a pathway that includes inflammation, repair, and remodeling. The pathway is more defined for tendons and consists of four discernable phases rather than three. The first phase is the creation of tendon fibers. These fibers turn into uncalcified fibrocartilage then into calcified cartilage. Healing ends when the tendon re-attaches onto the bone. The process of tendon re-growth usually requires twelve weeks and is influenced by factors such as tissue type, tissue vasculature, bone quality, health, and surgery type. (18)

\subsection{Types of Suture Anchors}

There are two major classifications of suture anchors commonly used for rotator cuff surgery, nonscrew and screw. (24) Screw anchors have a threaded portion and are mechanically dug into the bone for fixation. Non-screw anchors use an interference fit mechanism to be held in place (Fig 1-6).

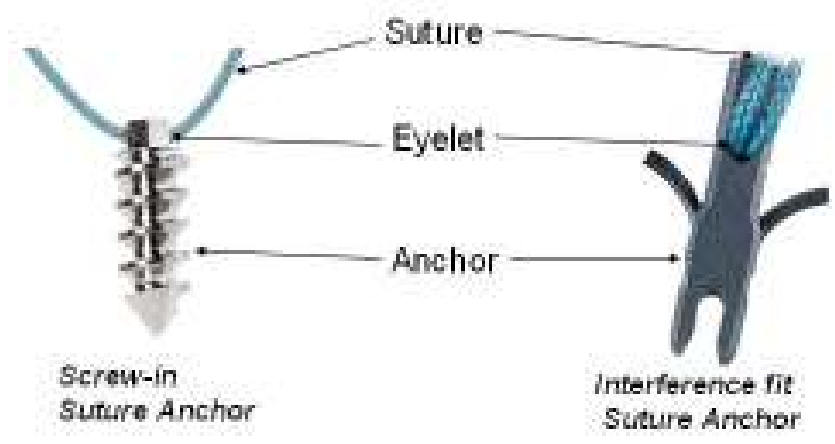

Figure 1-6: Screw type (left) and non-screw type (right) suture anchors.

Suture anchors can also be biodegradable. The ability to incorporate into the body offers many advantages but a major drawback is that these anchors have significantly lower pullout strengths than non-biodegradable because they are primarily made of a softer material. 


\subsection{Failure Modes of the Suture Anchor Technique}

The goal of the suture anchor is to maintain the tendon-bone interface until tendon reattachment has occurred. The anchor withstands tensile forces that can cause a multitude of failures at the anchor, suture, or tendon. A vulnerable area is the collection of sutures that thread through the anchor eyelet and connect to the tendon. These sutures can cut through bone tunnels, break, rip the tendon, and slip at the knot. Another failure site is the suture anchor. It could fail by pullout from the bone, loosening over time, or eyelet fracture. The scope of this study will focus only on the pullout failure of suture anchors.

\subsubsection{Factors Affecting Pullout Force - Bone}

The percentages of trabecular and cortical bone have been shown to influence the pullout strength of suture anchors. When an anchor is placed inside cortical bone it has a higher pullout force than when placed in trabecular bone. One study has found that the proximal portion of the humerus is composed of a higher total trabecular and cortical bone mineral density than that of the distal section resulting in higher loads to failure. (1)

Anchors inserted into the lesser tuberosity displayed higher loads to failure than when inserted into the greater tuberosity. Although the lesser tuberosity shows higher loads to failure, it is not an ideal implantation site because of the difficulty of access during repair and the distance from the supraspinatus tendon. This limits the implantation site to the greater tuberosity. There is considerable pullout force variability in the greater tuberosity as well. One study found that the pullout strength is $40 \%$ less in the anterior portion of the greater tuberosity in contrast to the posterior portion. (1) 
Disease states of bone also influence the pullout strength of suture anchors. Osteoporosis, for example, decreases the bone mineral density in both cortical and trabecular sections. When suture anchors are placed in lower density osteoporotic bone the pullout forces are lower.

\subsubsection{Factors Affecting Pullout Force - Suture Anchors}

The design of a screw type suture anchor has a couple variables. There are factors such as the pitch of thread, thread depth, major diameter, minor diameter, length, and shaft diameter (Fig 1-7). The variation of any of these factors has an effect on the pullout strength. A study put on by Chapman found that the major diameter of the screw, the length of engagement of the threads, and thread shape factor are the main contributing factors on pullout. $(18,5)$

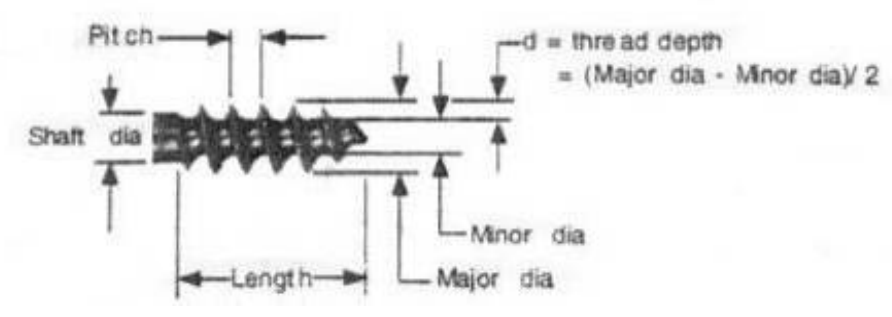

Figure 1-7: Suture anchor features.

A study by Ammon looked at the difference in pullout based on the type of suture anchor. It was found that the screw type suture anchors failed at higher pullout loads in comparison with the non-screw types. (1) 


\subsubsection{Factors Affecting Pullout Force - Suture Anchor Placement}

Due to the complexity of the glenohumeral joint with the various overlapping muscles, the surgeons are limited to the greater and lesser tuberosity of the humeral head for anchor placement.(1) Since the higher cortical thickness provides better pullout strength, placing anchors as far distal from the tip of the greater tuberosity will ensure the placement inside a high cortical thickness area. One study found that the insertion of anchors medial to the tip of the greater tuberosity, in proximal-anterior and proximalmiddle regions would be optimum.(1)

Another study investigated three different anchor insertion depths, deep, standard, and proud, to evaluate the effect on pullout strength (Fig. 1-8). 4 out of 11 standard and 6 out of 9 proud anchors failed early during the cyclic testing. The deep anchor had a higher failure load, around 160 newtons, and ultimate strength than standard or proud. The proud had early catastrophic failure caused by the mechanical weakening of the suture at the eyelet. (4)
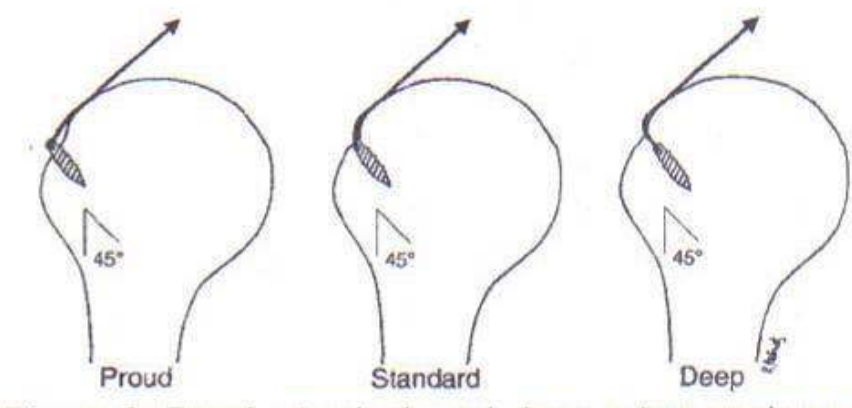

Figure 1-8: Suture anchor insertion depth Study by Bynum (4).

The angle to which the anchor is inserted into the bone has also been examined at. The preferred angle of implantation of the suture anchor has been found to be 45 degrees 
to the axis of the humerus. As per the importance of the angle of insertion, one study found the load to failure was affected significantly by the angle of implantation between the bone and anchor. The pullout force decreases as the angle of insertion increases. (18) It is common practice by surgeons to insert suture anchors at 45 degrees to the humeral axis. 


\section{CHAPTER 2 OBJECTIVES}

The primary objective of this study is to understand and model the effects of frontal plane humeral elevation and anchor type on the pullout strength of suture anchors implanted into replica humeral bone. Understanding how these factors influence pullout forces could eventually play a key role in the rehabilitation capabilities of rotator cuff injuries.

The secondary objective is to validate the test methodology of suture anchor pullout. Having a robust test method will ensure the outcomes of the pullout tests can be compared to previous findings reliably.

The following hypotheses presented for this study are:

Hypothesis 1: An increase in humeral frontal elevation angle will decrease the pullout force of suture anchors because it is approaching a uniaxial direction of pull, which is the worst-case condition.

Hypothesis 2: The elevation angle of the humerus will have a greater effect on the pullout force than the choice of suture anchor because it controls the direction of pull. 


\section{CHAPTER 3 METHODS AND MATERIALS}

\subsection{Suture Anchor Pullout from Polyurethane Blocks Test Setup}

The pullout of suture anchors inserted into polyurethane blocks was done as a validation to the testing methodology of anchor pullout. To verify this, the results were compared with the results from a similar study done by Nien. This study investigated the pullout forces of two suture anchors implanted into two different density blocks.

The prior study by Nien investigated the pullout force of suture anchors using a polyurethane foam block with a density of $0.128 \mathrm{~g} / \mathrm{cm}^{3}$ (or $7.991 \mathrm{~b} / \mathrm{ft}^{3}$ ) because it closely matched the density of the humeral head (10). Two $13 \mathrm{X} 18 \mathrm{X} 4 \mathrm{~cm}$ polyurethane blocks were obtained with densities of $5 \mathrm{lb} / \mathrm{ft}^{3}$ and $10 \mathrm{lb} / \mathrm{ft}^{3}$ from SAWBONES for this study. These blocks were chosen to capture the influences of block density on the pullout force, as well as compare with the results found from the Nien study using a linear interpolation.
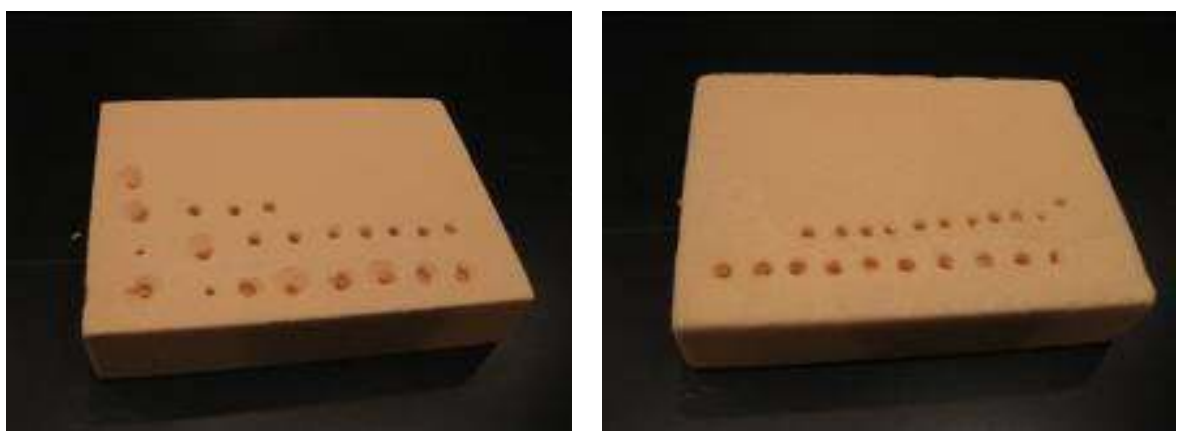

Figure 3-1: SAWBONES polyurethane $10 \mathrm{lb} / \mathrm{ft}^{3}$ (left) and $5 \mathrm{lb} / \mathrm{ft}^{3}$ (right) density blocks.

Two types of anchors, a 5mm PLA (poly lactic acid) biodegradable Mitek Spiralok $^{\mathrm{TM}}$ and $6.5 \mathrm{~mm}$ titanium Smith \& Nephew Twinfix ${ }^{\mathrm{TM}}$ anchor, were chosen to be pulled out of the polyurethane blocks. These anchors were selected to discriminate differences in pullout based on anchor material and size. In comparison, the study by 
Nien used a 1/4-20 standard metallic screw. Table 3-1 and figure 3-2 below show the comparison of the anchors used in this study against the ones used in the Nien study.

Table 3-1: Suture anchors used in the pullout tests from this study as well as the study by Nien.

\begin{tabular}{|c|c|c|}
\hline Suture Anchor & Major Diameter & Material \\
\hline Smith \& Nephew Twinfix $^{\mathrm{TM}}$ & $6.5 \mathrm{~mm}$ & Titanium \\
\hline Mitek Spiralok $^{\mathrm{TM}}$ & $5 \mathrm{~mm}$ & PLA \\
\hline $1 / 4-20$ Standard Screw (Nien) & $6.35 \mathrm{~mm}$ & Steel \\
\hline
\end{tabular}

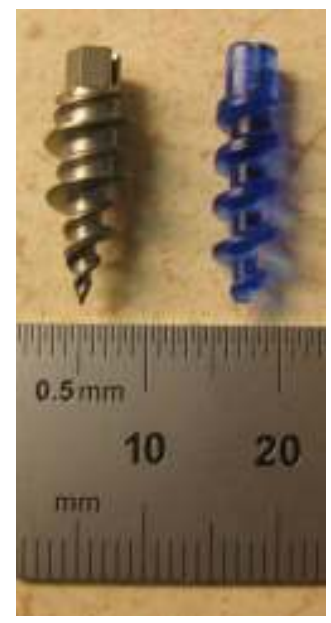

Figure 3-2: 6.5mm Smith \& Nephew Twinfix (left) and 5mm Mitek Spiralok (right) suture anchors.

The anchors were inserted into the polyurethane foam block perpendicular to the surface using the attached delivery system. The anchor was implanted up to the top level of the threads and the delivery system was removed leaving a series of sutures through the anchor eyelet. The polyurethane block was clamped using $\mathrm{C}$ clamps to the base of an Instron Micro Tensile Tester such that the anchor was in line with the direction of pull. The uniaxial pull was also chosen to match the pullout methods by Nien. The sutures were thread through a wire clamp adapter and tightened but not pulled taught. The instron was jogged vertically until a tension was seen on the digital output. Figure 3-3 below shows the test setup. 


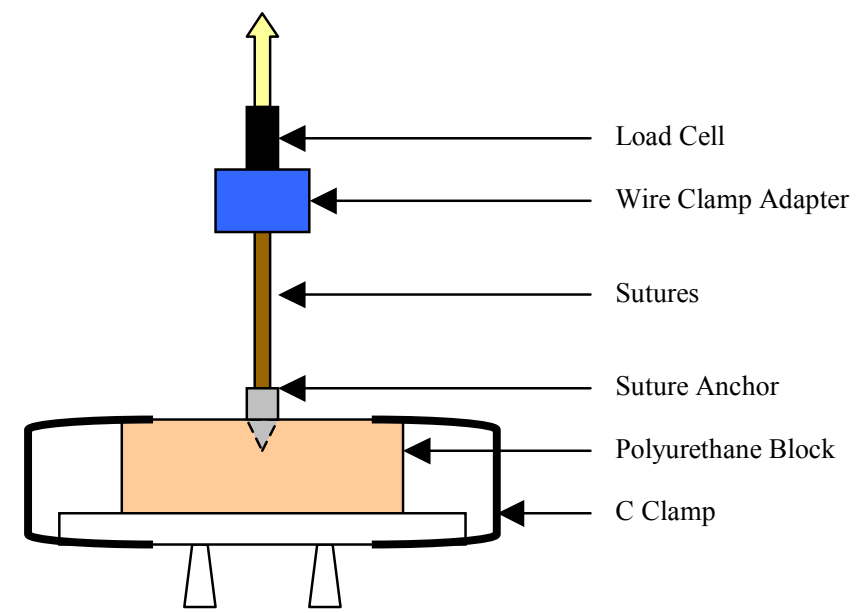

Figure 3-3: The uniaxial pullout of suture anchors test setup.

The suture anchors were pulled at a rate of $.15 \mathrm{~mm} / \mathrm{s}$ up to a maximum displacement of $1 \mathrm{~cm}$. The peak pullout forces were captured with a 50lbf load cell. The data was recorded into a portable pda device and later transferred to a computer for analysis. The system was reset and another anchor was placed into the foam $5 \mathrm{~cm}$ from the previous spot to eliminate any contributions from prior samples. A total of 10 trials were run per anchor per foam block for a total of 40 test runs.

\subsection{Humeral Frontal Plane Elevation Angle Effects on Pullout Forces Test Setup}

The test setup for the humeral angle evaluation analysis incorporated an adjustable bone holder, pulley system, and instron to investigate the pullout forces at various angles. These fixtures were able to orient the humerus at different angles while maintaining a pull force that was in line with the load cell of an instron.

Truncated humeri were obtained from SAWBONES as the test interface for the study (Fig 3-4). The SAWBONES humeri are composite replica bones made of a rigid 
foam shell and inner cancellous material. These distinctive regions give a comparable model to that of bone (Table 3-2).

Table 3-2: Density comparison of SAWBONES replica bone and actual humeral bone.

\begin{tabular}{|c|c|c|}
\hline & $*$ Cancellous Region & Cortical Region \\
\hline Sawbones Humeri & $0.128-0.192 \mathrm{~g} / \mathrm{cm}^{3}$ & $0.321-0.353 \mathrm{~g} / \mathrm{cm}^{3}$ \\
& & $(3-5 \mathrm{~mm} \mathrm{deep})$ \\
\hline Tingart Study & $0.12 \pm 0.03 \mathrm{~g} / \mathrm{cm}^{3}$ & $0.48 \pm 0.06 \mathrm{~g} / \mathrm{cm}^{3}$ \\
N=20 Cadaver (28) & & \\
\hline
\end{tabular}

*Majority of humeral head is cancellous bone.

$1-1 / 4$ " schedule $40 \mathrm{PVC}$ pipe was cut to a length of $100 \mathrm{~mm}$ and placed onto a polyurethane potting base. The distal end of the truncated Sawbones humeri was inserted on top of the protruding cylinder of the potting base. The bottom hole of the truncated humeri created an interference fit with the cylinder and the base of the bone was in line with the surface of the potting base.
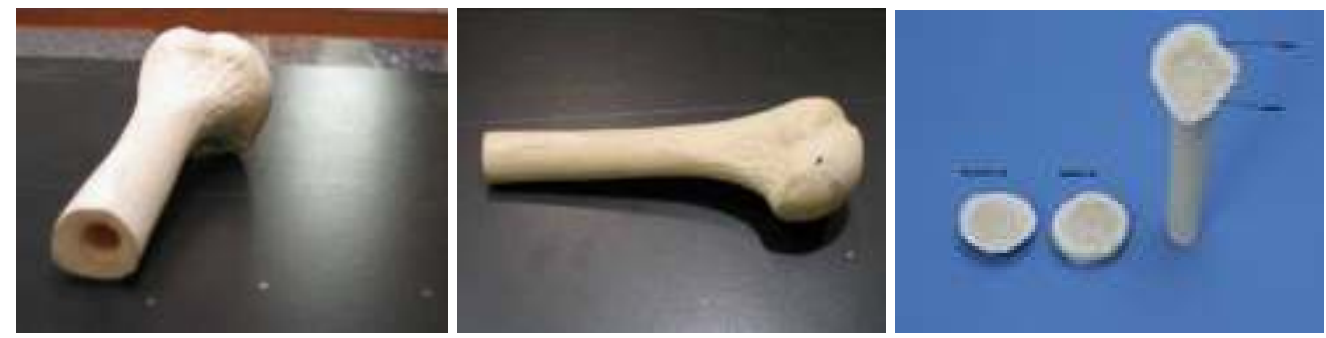

Figure 3-4: SAWBONES humeri with distal hole for placement onto potting base.

A polyurethane gluing mixture was used to create a bond between the replica bone and PVC pipe. This subassembly was created to decrease the amount of bending translated into the humeral body. It was also made to repeatedly orient the bones inside the holding fixture and keep them at a known distance and angle.

To create the polyurethane glue mixture, goggles, a lab coat, and heavy-duty gloves were needed because of the use of the sensitizing agent isocyanate. $30 \mathrm{~mL}$ of isocyanate was combined with $30 \mathrm{~mL}$ of polyol in a $100 \mathrm{~mL}$ beaker. As the mixture 
became viscous, after 3 to 4 minutes of stirring, it was poured into the PVC pipe and bone composite vacancy and distributed evenly. After a 15 minute cure the bond strength was verified by pulling on the bone.
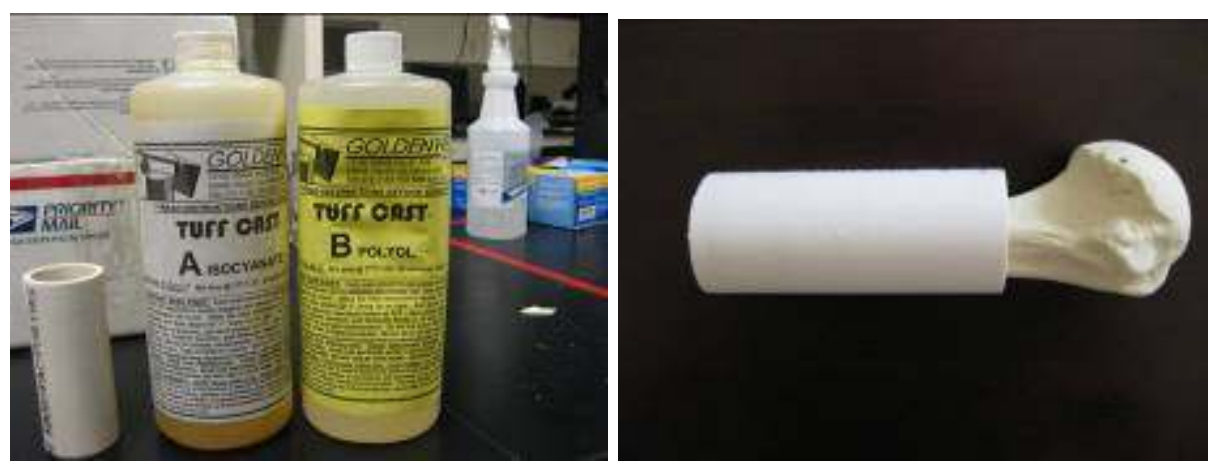

Figure 3-5: Isocyanate and Polyol solutions (left). Potted humerus subassembly (right).

3 different suture anchors were chosen to compare the effects of anchor type against angle of elevation in terms of pullout force. The suture anchors were chosen based on the differences in major diameter. There was a $6.5 \mathrm{~mm}$ Smith \& Nephew Twinfix ${ }^{\mathrm{TM}}$, 5mm Mitek Fastin RCTM, and 3.5mm Smith \& Nephew Twinfix ${ }^{\mathrm{TM}}$ (Fig 3-6).

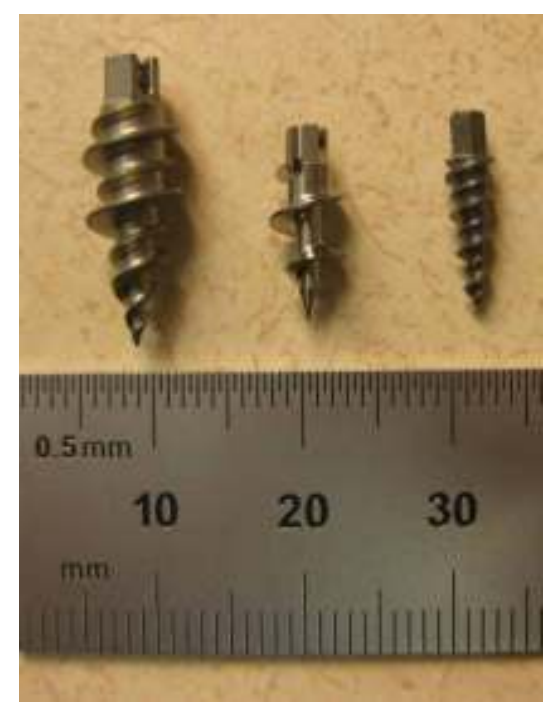

Figure 3-6: 6.5mm Smith \& Nephew Twinfix (left), $5 \mathrm{~mm}$ Mitek Fastin RC (middle), and 3.5mm Smith \& Nephew Twinfix (right) suture anchors. 
As per common clinical procedures, anchors were inserted using their attached delivery systems into the greater tuberosity of the humeral head at an angle of 45 degrees to the body axis of the humerus. They were threaded to a depth such that the top of the anchor was flush with the bone material.

The bone/pve composite was then inserted into the holding fixture and locked into a fixed distance by a proximal top set screw. A soldered steel cable wire (type 304 stainless steel wire rope, 1 X7 strand, .027 " diameter) was inserted through the eyelet of the anchor. The wire was chosen because of its breaking strength and resistance to elongation so as not to fail during the test run or add variability to the pullout.

The holding fixture was created to lock the bone assembly in fixed angles. The angle of elevation was defined as the angle between the direction of pull and the centerline of the humeral body. The fixturing had hypothetical angles of elevation at 120 , 150 , and 180 degrees but in reality were calculated as 111,141 , and 177 with the offset of the suture anchor from the centerline and mounted pulley dimensions. These angles were chosen to replicate the range of motion the arm would experience during normal frontal elevation. With three points the effect of angle elevation on the pullout force could be studied (Fig 3-7).

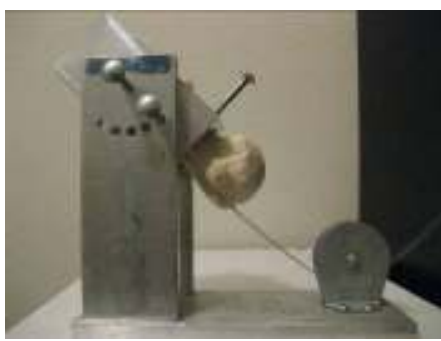

(a)

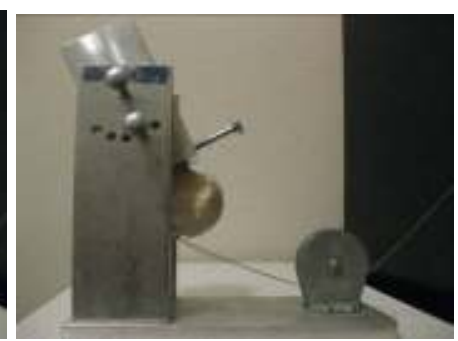

(b)

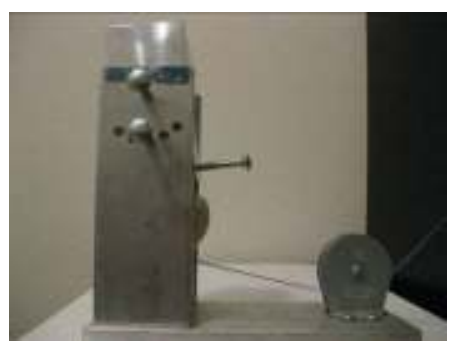

(c)

Figure 3-7: Potted humeral bone subassembly in bone holding fixture for hypothetical humeral frontal plane angle evaluations (a) $180^{\circ}$, (b) $150^{\circ}$, and (c) $120^{\circ}$. 
The wire was fed through the mounted pulley, and then through the pulley fixture at the top of an Instron In-Spec $2200^{\mathrm{TM}}$ tensile tester (Fig 3-8). The holding fixture was then locked into a set angle by four setscrews located at the middle and front end of the rotating holder.

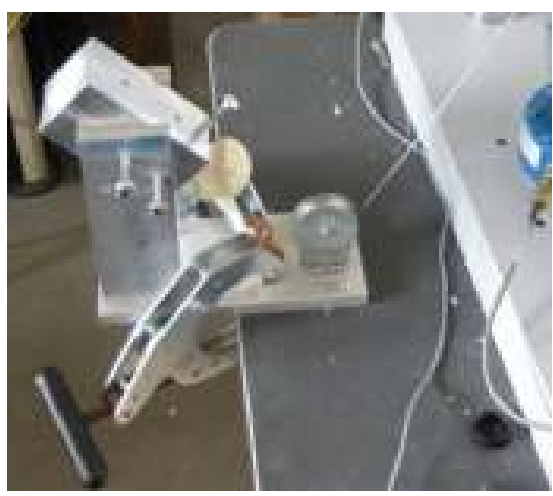

(a)

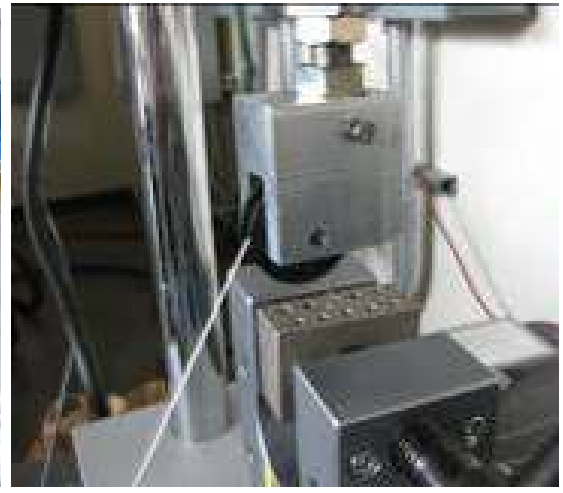

(b)

Figure 3-8: Experimental setup of potted humeral bone subassembly in bone holding fixture to investigate the effects of humeral bone elevation and suture anchor type on the pullout strength.

The wire was pulled taught and clamped into the bottom grip of the instron. A visual inspection ensured that the wire was in line with the load cell and top pulley. The bone holding fixture was clamped to the table near the mounted pulley so as to prohibit rotation of the system during the test. The program was set to pull at a rate of $0.5 \mathrm{~mm} / \mathrm{s}$ for a maximum displacement of $5 \mathrm{~cm}$. Forces were outputted from the tensile tests. A total of 3 different suture anchors were tested at three different degrees of elevation in order to map the suture anchor pullout force against the humeral bone elevation angle to see any trends or effects.

\subsection{Statistical Methods}

The JMP Statistical software was used for the analysis of data for this study. For the uniaxial tests, the suture anchors were grouped according to type and the 
polyurethane blocks were grouped according to density. A Student's t-test was performed to evaluate significant pullout force differences between block density and suture anchors. An F test was used to see the probability that the two distributions being examined are statistically similar. F ratios were calculated to examine the contribution of each source (anchor, angle, density, and interactions) on the variance seen in the model. The use of the Tukey HSD (Honesty Significant Difference) analysis was also used to compare all possible pairs of means for the three anchors of the angle evaluation. This method distinguishes differences between groups if the difference between their means is greater than the standard error. Leverage plots were created to visualize the confidence intervals of the $\mathrm{f}$ tests and show if a factor had a significant effect on the model. A least squares means was used because each test setup had more than one effect.

A design of experiments (DOE) was preformed to populate a run order of three different suture anchors and three different frontal elevation angles. This DOE, which can be found in the appendix, was able to achieve 100\% confidence that the pullout force model can be described by both the anchor type as well as humeral elevation angle.

ANOVA analysis as well as effects tests were run to understand the effects of the anchor and angle on pullout force. Parameter estimates were calculated to provide the equation for pullout force based on elevation angle. A least squares means analysis was also run to determine the significant differences between anchors. 


\section{CHAPTER 4.0 RESULTS}

\subsection{Pullout Strength of Suture Anchors Implanted into Polyurethane Blocks}

Peak pullout force data was collected from forty pullout tests using two different density (5 and 101b) polyurethane blocks and two different suture anchors $(6.5 \mathrm{~mm}$ Smith $\&$ Nephew and 5mm Mitek). The purpose of these tests was to verify suture anchor pullout methodology to ensure comparable results with previous work. The individual test graphs can be seen in the appendix. Table 4-1 shows the peak pullout forces from these forty tests. In all cases, the failure mode was suture anchor pullout.

Table 4-1: Comparison of the peak pullout forces of a $6.5 \mathrm{~mm}$ Smith \& Nephew and a $5 \mathrm{~mm}$ Mitek suture anchor implanted into 5 and $10 \mathrm{lb}$ density polyurethane blocks.

\begin{tabular}{|c|r|r|r|r|}
\hline & \multicolumn{3}{|c|}{ Pullout Force (N) } \\
\hline & \multicolumn{2}{|c|}{ 5lb } & \multicolumn{1}{c|}{ 10lb } \\
& \multicolumn{1}{|c|}{ Density Block } & \multicolumn{1}{c|}{ Density Block } \\
\hline Run & Smith & \multicolumn{1}{c|}{ Mitek } & \multicolumn{1}{c|}{ Smith } & \multicolumn{1}{c|}{ Mitek } \\
\hline 1 & 77.338 & 72.715 & 210.214 & 171.688 \\
\hline 2 & 85.189 & 71.645 & 218.541 & 176.923 \\
\hline 3 & 86.379 & 68.671 & 199.731 & 178.707 \\
\hline 4 & 90.202 & 70.693 & 211.302 & 166.097 \\
\hline 5 & 84.492 & 71.169 & 216.060 & 150.514 \\
\hline 6 & 76.998 & 65.935 & 213.681 & 190.484 \\
\hline 7 & 86.633 & 67.005 & 209.517 & 193.934 \\
\hline 8 & 87.823 & 68.314 & 214.989 & 191.792 \\
\hline 9 & 89.607 & 65.935 & 205.473 & 198.930 \\
\hline 10 & 82.827 & 63.793 & 210.469 & 191.673 \\
\hline & $\mathbf{8 4 . 7 4 9}$ & $\mathbf{6 8 . 5 8 8}$ & $\mathbf{2 1 0 . 9 9 8}$ & $\mathbf{1 8 1 . 0 7 4}$ \\
\hline Mean & & & & $\mathbf{1 5 . 1 6 3}$ \\
\hline Deviation & $\mathbf{4 . 5 7 0}$ & $\mathbf{2 . 9 2 7}$ & $\mathbf{5 . 4 4 0}$ & \\
\hline
\end{tabular}

Shown in Figure 4-1 below, the summary of fit data shows the test setup had an rsquared of $98 \%$ meaning all factors contributing to the variability in the model were caught. Based on the ANOVA there was an F Ratio of 684.1887 as well as a Prob $>$ F less 
than 0.0001 indicating a strong presence of variables that can explain the pullout force response.

The effects test verifies that both the anchor type and block density have a significant effect on the model based on a Prob $>F$ being less than 0.0001 . Block density was shown to have a greater effect on the model than the suture anchor from a comparison of F Ratio values of 1972.5 and 73.5 respectively. The F Ratio was also able to show that the interaction between the anchor and polyurethane block had a negligible effect on the model with a value of 6.6 .

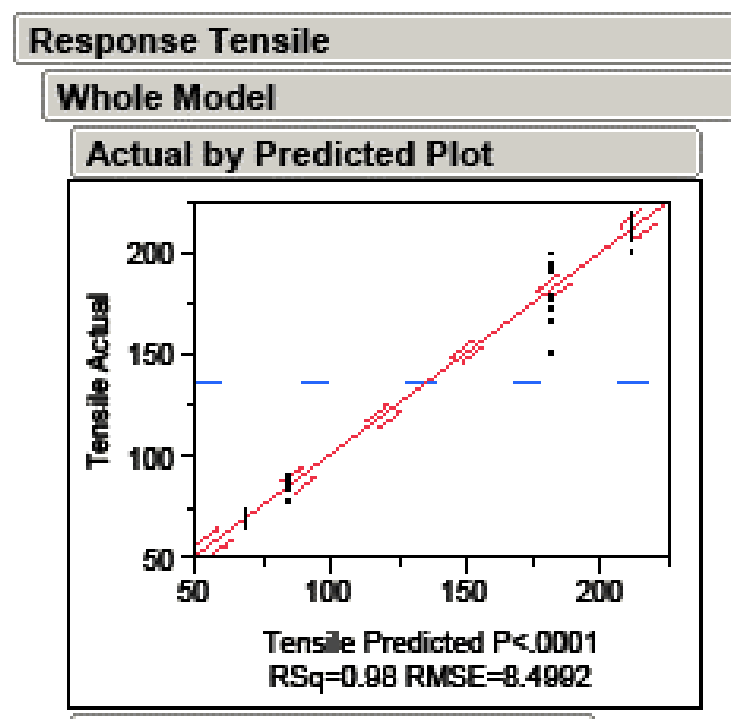

Figure 4-1: The whole model test for effects of suture anchor pullout in polyurethane blocks. There is a high test significance in capturing the variables that have an effect on pullout strength, $R S q=0.98$.

Figure 4-2 shows the difference between the 5 and $10 \mathrm{lb}$ density blocks. Block density had a significant effect on the model as seen by the intersection of the blue line with the red line from the leverage plot. The 5lb block had a least squares mean of $76.67 \mathrm{~N}$ and the $10 \mathrm{lb}$ block had a least square mean of $196.04 \mathrm{~N}$. This is a difference of 
119.37N. The Student's t-test was able to show a significant difference on pullout force between the $10 \mathrm{lb}$ and $5 \mathrm{lb}$ density blocks.

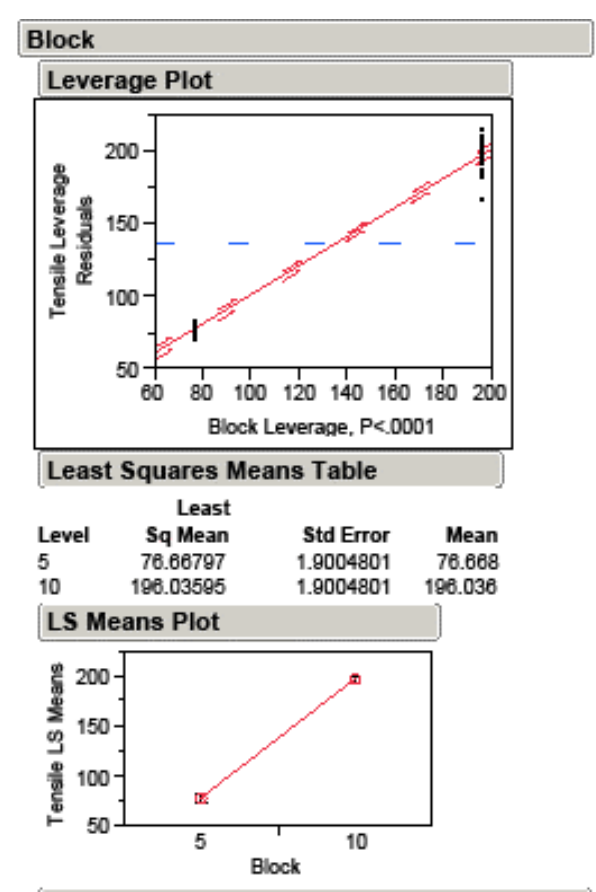

Figure 4-2: Significance of the block density effect on suture anchor pullout. Block density leverage plot indicates a very strong effect on the pullout force. The 10lb density block had higher pullout forces than the 5lb block.

Figure 4-3 compares the difference between the suture anchors and their effect on the pullout force. The leverage plot verifies that the suture anchor has an effect on the system with the intersection of the red line across the blue line. The Mitek and Smith \& Nephew anchors had least squares means of $124.831 \mathrm{~N}$ and $147.873 \mathrm{~N}$ respectively. This is a percent difference of $15.6 \%$. The student's $t$ test was able to show a significant difference between the Mitek and Smith \& Nephew suture anchors on pullout force. 


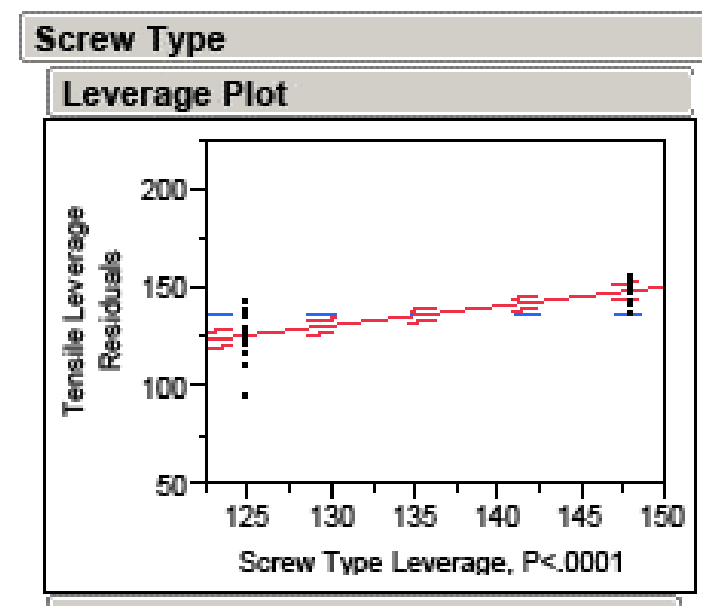

Figure 4-3: Significance of the suture anchor effect on suture anchor pullout. Suture anchor type leverage plot indicates a strong effect on the pullout force. The $6.5 \mathrm{~mm}$ Smith\&Nephew had slightly higher pullout forces than the $5 \mathrm{~mm}$ Mitek anchor.

The interaction between the suture anchor and density of the polyurethane block is shown to have an effect on the model as seen from the leverage plot in Figure 4-4. The Smith \& Nephew anchor in the 10lb block had the highest least squares mean pullout force of $211 \mathrm{~N}$, whereas the Mitek anchor in the 5lb block had the lowest least squares mean pullout force of $69 \mathrm{~N}$. The Student's t-test was able to show that the four anchor and density configurations were significantly different from one another.

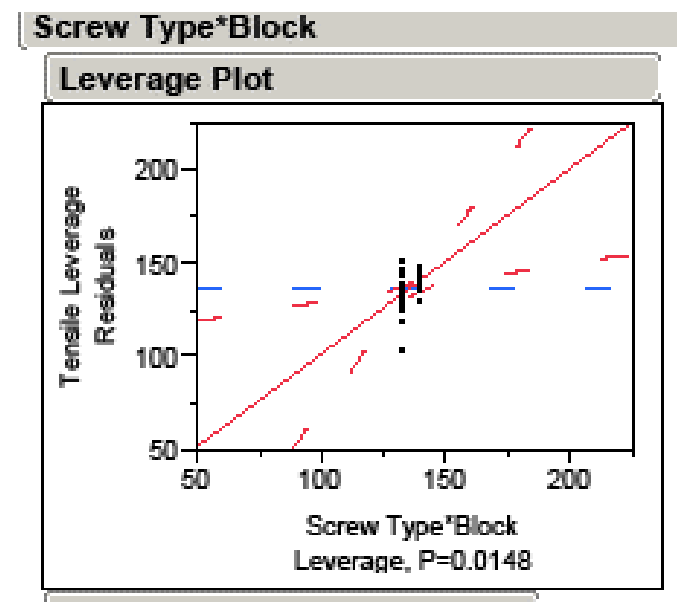

Figure 4-4: Significance of the block density and suture anchor interaction effect on suture anchor pullout. The interaction between block density and suture anchor leverage plot indicates a borderline effect on pullout force. 
The statistical analysis above ensured the variables of suture anchor and block density were isolated and the data was reliable. These results were then compared with the results of the study by Nien to observe their relevance to prior published work. A linear interpolation using the 5 and $101 \mathrm{~b}$ density block pullout data gave the pullout force values in an 81 density block. Table 4-2 shows the calculated forces and Nien results.

Table 4-2: Comparison of suture anchor pullout forces in an 8lb density polyurethane block.

\begin{tabular}{|l|c|c|c|}
\hline \multicolumn{1}{|c|}{ Suture Anchor } & \multicolumn{3}{|c|}{ Pullout Force (N) } \\
\hline 6lb Block Density & $\begin{array}{c}\text { 10lb Block } \\
\text { Density }\end{array}$ & $\begin{array}{c}\text { 8lb Block } \\
\text { Density }\end{array}$ \\
\hline 5mmMitek \& Nephew & $84.749 \pm 4.570$ & $210.998 \pm 5.440$ & 160.5 \\
\hline $\begin{array}{l}\text { Standard 1/4-20 Screw } \\
\text { (Nien) }\end{array}$ & $68.588 \pm 2.927$ & $181.074 \pm 15.163$ & 136.1 \\
\hline
\end{tabular}

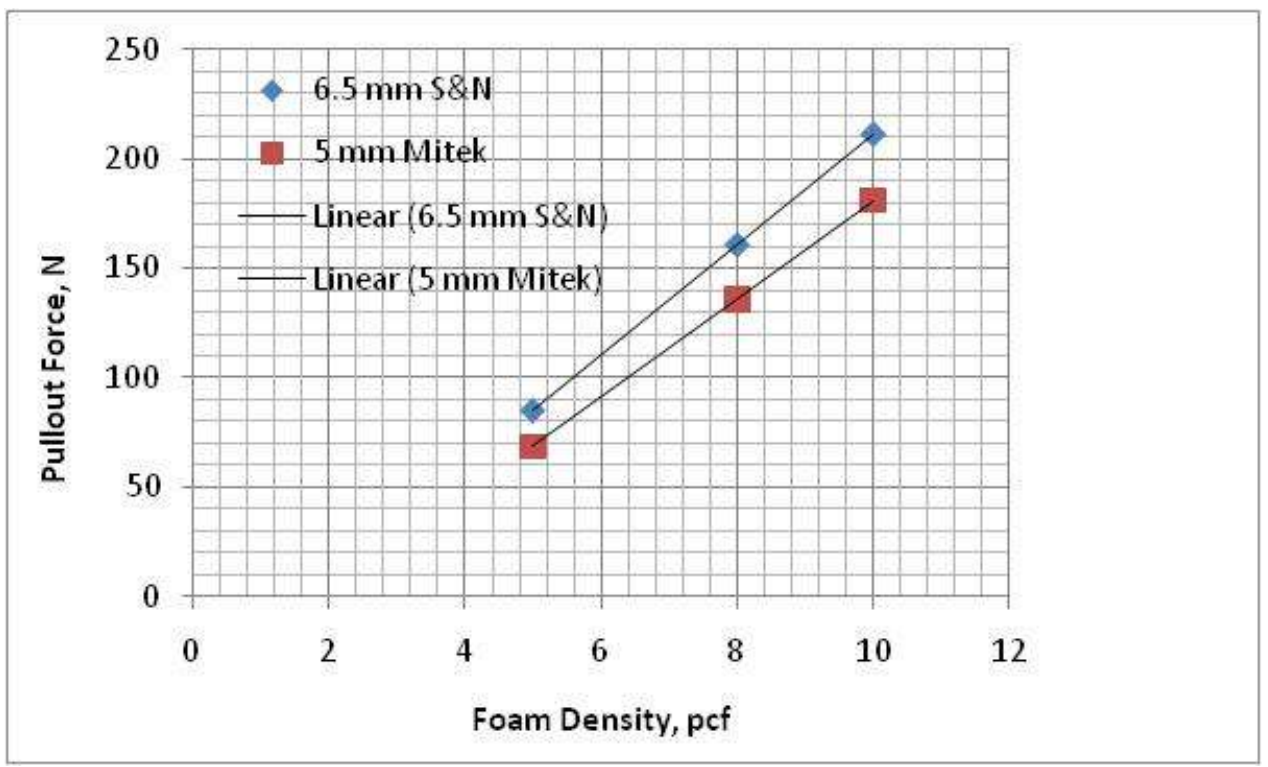

Figure 4-5: Linear interpolation of 6.5mm Smith \& Nephew and $5 \mathrm{~mm}$ Mitek suture anchors to obtain pullout force data in an 81b density polyurethane block.

The comparison between the results of this study with Nien's results is positive.

There is a $3 \%$ difference in pullout between the $6.5 \mathrm{~mm}$ Smith \& Nephew anchor and the 1/4-20 screw, which are relatively close in size and material. There is a larger difference, 
$12 \%$, between the $5 \mathrm{~mm}$ Mitek and the $1 / 4-20$ screw, but this is expected because of their material and size differences.

\subsection{Humeral Frontal Elevation Angle and Suture Anchor Effects on Pullout Forces}

A total of 18 pullout tests were performed using three different suture anchors at 3 different frontal elevation angles to examine the influences and contributions of elevation angle and anchor type on suture anchor pullout force (Table 4-3). All tests failed by anchor pullout.

Table 4-3: Pullout forces of 3 different suture anchors at 3 different frontal elevation angles.

\begin{tabular}{|c|c|c|c|c|c|c|c|}
\hline Test & Angle & Anchor & Pullout Force (N) & Angle & & Anchor & \\
\hline 1 & 3 & 3 & 223.7 & 1 & 111.88 & 1 & 6.5mm Smith\&Nephew \\
\hline 2 & 3 & 2 & 297 & 2 & 140.72 & 2 & $5 \mathrm{~mm}$ Mitek \\
\hline 3 & 2 & 3 & 378.4 & 3 & 176.9 & 3 & 3.5mm Smith\&Nephew \\
\hline 4 & 1 & 3 & 464.9 & & & & \\
\hline 5 & 2 & 2 & 597.7 & & & & \\
\hline 6 & 2 & 1 & 838.57 & & & & \\
\hline 7 & 2 & 1 & 984.33 & & & & \\
\hline 8 & 1 & 1 & 1012.44 & & & & \\
\hline 9 & 3 & 2 & 359.2 & & & & \\
\hline 10 & 1 & 2 & 754.91 & & & & \\
\hline 11 & 2 & 3 & 327.3 & & & & \\
\hline 12 & 1 & 2 & 621.48 & & & & \\
\hline 13 & 1 & 3 & 503 & & & & \\
\hline 14 & 2 & 2 & 442.21 & & & & \\
\hline 15 & 3 & 1 & 599.5 & & & & \\
\hline 16 & 3 & 3 & 192.8 & & & & \\
\hline 17 & 3 & 1 & 612 & & & & \\
\hline 18 & 1 & 1 & 1031.31 & & & & \\
\hline
\end{tabular}

From figure 4-6, the summary of fit shows an R-squared value of $96 \%$, which means the DOE analysis was able to accurately profile the model and catch the variables that contribute to the variation. The regression plot shows the larger size suture anchors 
had larger pullout forces. The $6.5 \mathrm{~mm}$ Smith \& Nephew anchor had the highest pullout force over the angle range, the $5 \mathrm{~mm}$ Mitek anchor had the second highest, and the $3.5 \mathrm{~mm}$ Smith \& Nephew anchor had the lowest. The regression plot also shows that as the angle of elevation is increased, the pullout force decreases.

The ANOVA of the entire model resulted in an F Ratio of 63.4 showing that there are detectable variables that can explain the pullout force. The lack of fit analysis demonstrated that there was no need to add more interaction terms to increase the fit of the model. Since the r-squared value from the summary of fit was high and the F Ratio from the lack of fit was small the fit cannot be improved by adding more interaction terms. 


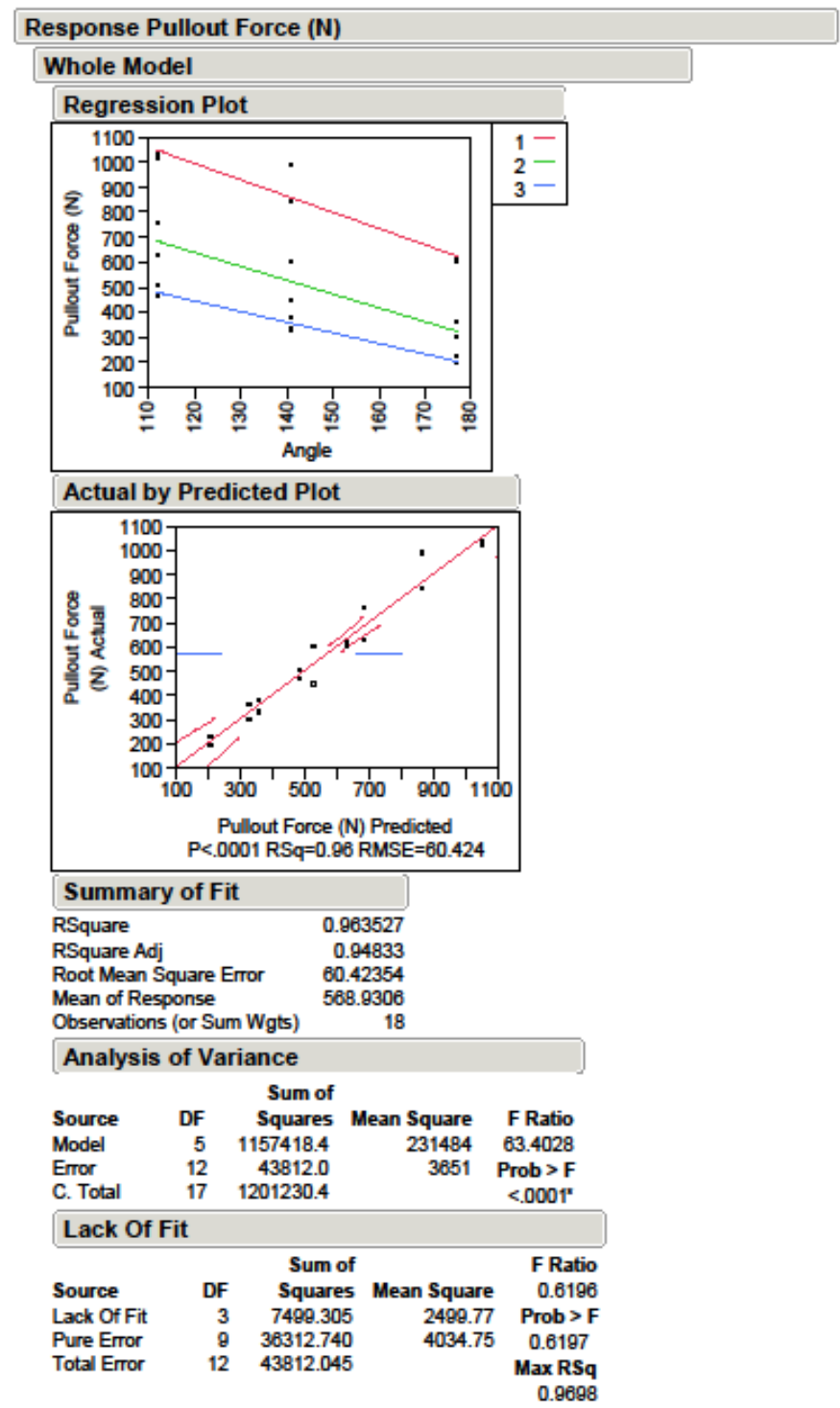

Figure 4-6: The whole model test for effects of suture anchor pullout at various elevation angles. There is a high test significance in capturing the variables, angle of elevation and suture anchor type, that have an effect on pullout strength, $\mathrm{RSq}=\mathbf{0 . 9 6}$. An increase in elevation angle decreases the pullout force and an increase in suture anchor size increases the pullout force.

From the effects test both the anchor and angle were shown to have an effect on the model with $\mathrm{F}$ ratios of 105.88 and 102.28 respectively (Fig 4-7). The relatively equal F Ratios between the suture anchor and elevation angle show that they have an equal effect on the pullout force. In comparison, the interaction between the anchor and the angle did not have an effect on the model with an F Ratio of 1.490. 


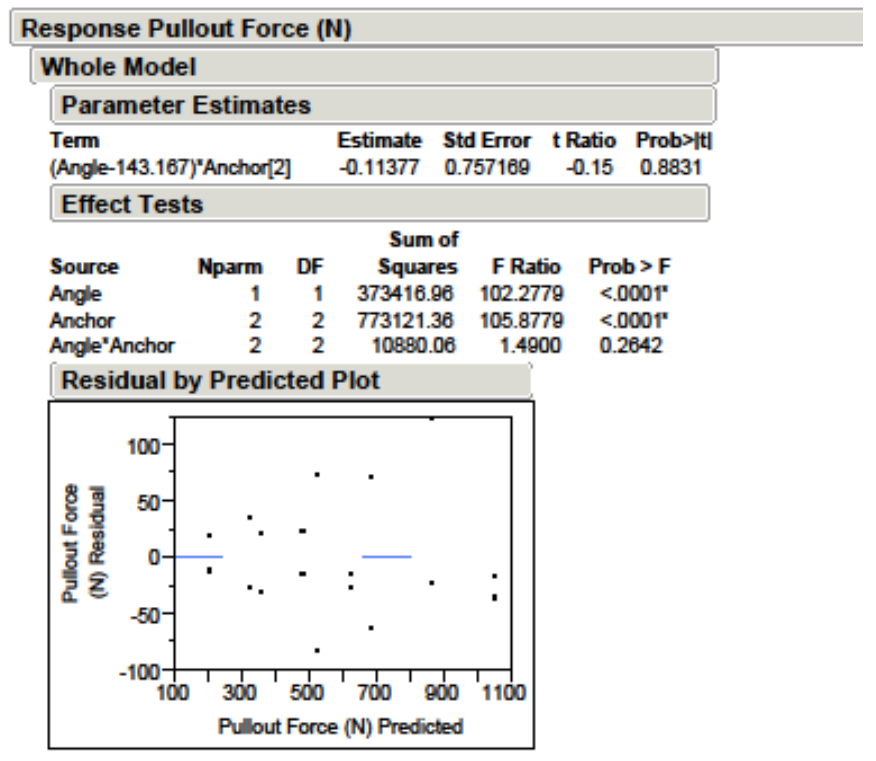

Figure 4-7: Contributions of the variables, anchor and angle, on the pullout force from the effects test. The angle and anchor have relatively similar effects on the pullout forces, $F$ Ratios.

The leverage plots shown in figure 4-8 verify that the anchor and angle have a significant effect on the model by the intersection of the red line across the blue line. These graphs also verify that the pullout force increases with increasing suture anchor size as well as decreasing elevation angle. Since there were multiple effects on the pullout force, the least squares means table was created as seen below. Anchor 1 had the highest least squares mean pullout force of $846.39 \mathrm{~N}$, anchor 2 was in the middle at $512.08 \mathrm{~N}$, and anchor 3 was the smallest at $348.35 \mathrm{~N}$. The Tukey HSD analysis of the anchors shows significant differences between the three suture anchors. 

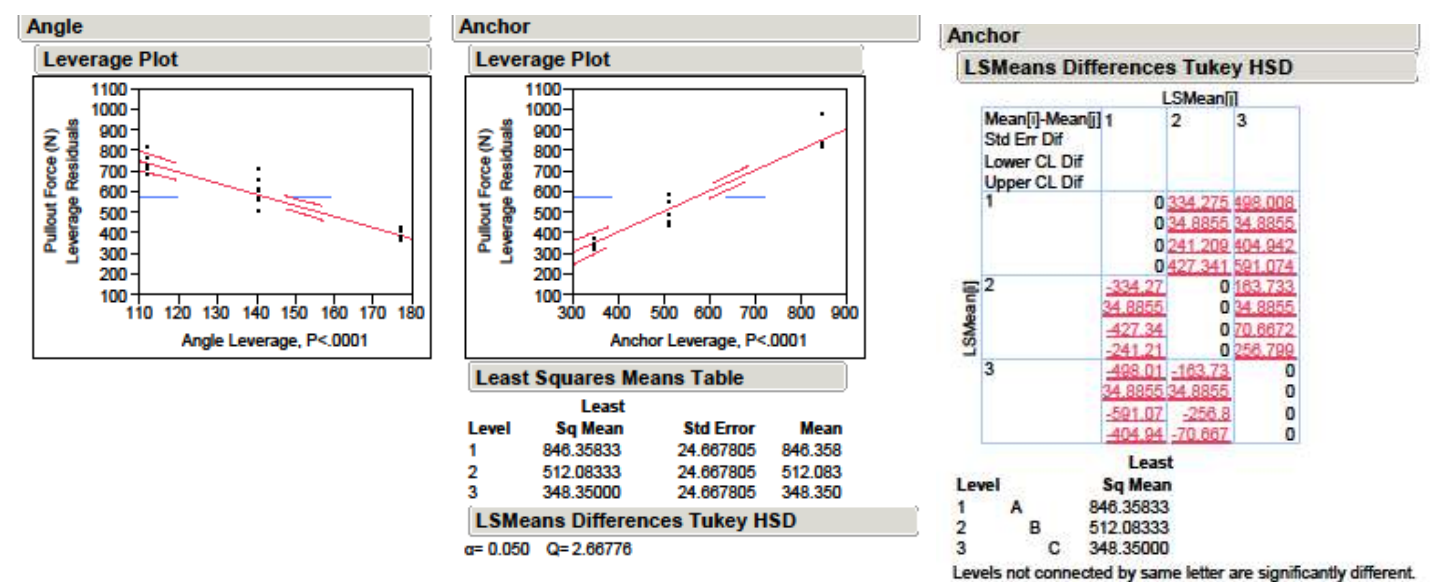

Figure 4-8: Significance of the elevation angle as well as anchor type effect on suture anchor pullout. Angle leverage plot and anchor leverage plot both indicate a strong effect on the pullout force. An increase in elevation decreases the pullout force. A higher size anchor increases the pullout force.

The parameter estimates calculated of the suture anchors and angles were used to create a prediction expression. This expression was able to relate the pullout force with the elevation angle per suture anchor. From this formula the pullout force for each anchor can be modeled as:

6.5mm Smith \& Nephew: Force $=-6.48 *$ Angle +1774.67

5.0mm Mitek: Force $=-5.52 *$ Angle +1303.591

3.5mm Smith \& Nephew: Force $=-4.23 *$ Angle +954.6

The above equations show the contributions of anchor size and elevation angle on pullout force. In each equation the angle term is negative indicating a decrease in pullout force with an increase in elevation angle. The magnitudes of the y intercepts relate with the size of suture anchor. The largest suture anchor had the highest y intercept and the smallest anchor had the smallest y intercept. 


\section{CHAPTER 5.0 DISCUSSION}

\section{1 Effects of Elevation Angle and Suture Anchor Choice on Pullout Force}

The primary objective of this study was to test the effects of humeral arm frontal elevation angle on the pullout force of suture anchors. One hypothesis posed was that as the elevation angle increases in the frontal plane, the force needed to pullout a suture anchor will decrease because it approaches a path of least resistant perpendicular pull.

The testing was able to verify this hypothesis by showing that as the angle of elevation increases, the force to pullout a suture anchor decreases. This was likely due to the fact that the suture anchor was being pulled into the humeral head at lower elevation angles. As the angle of elevation increased the direction of pull approached a perpendicular force relative to the bone surface, which is considered the worst-case condition for integrity because it is the path of least resistance.

The second hypothesis of the study was the comparison of effects on pullout strength between the suture anchor type, in terms of size, and angle of elevation. The hypothesis stated that the angle of humeral elevation in the frontal plane would have a greater effect on the pullout force than the choice of anchor because it controls the direction of pull.

This hypothesis was contradicted by this study with the testing of 3 different suture anchors at 3 different elevation angles. The results showed that both the angle of elevation of the humerus as well as the anchor type have a significant impact on the pullout force of suture anchors. The results also showed that the contributions on variance seen in the model were equal between the angle of elevation and anchor. Suture anchor 
selection is as important as angle elevation for understanding the risk of suture anchor pullout.

The study showed that the higher size suture anchors provided the higher pullout forces. This is likely due to the increased surface area contact of the suture anchor threads providing resistance to pullout. This idea is supported in the results. Based on the least squares means the $5 \mathrm{~mm}$ Mitek anchor was $330 \mathrm{~N}$ less than the $6.5 \mathrm{~mm}$ Smith \& Nephew anchor and the $3.5 \mathrm{~mm}$ Smith \& Nephew anchor was $500 \mathrm{~N}$ less in terms of pullout force.

In terms of rehabilitation, the size of suture anchor contributed to the traumatic failure of the interface. It was observed that the pullout of the $6.5 \mathrm{~mm}$ Smith \& Nephew anchor produced large areas of bone damage. In comparison, the smallest $3.5 \mathrm{~mm}$ Smith $\&$ Nephew anchor produced a clean and less traumatic pullout. There is a clear tradeoff between the pullout strength and traumatic failure. The larger anchors provide higher pullout forces, but also more destructive failures.

\subsection{Pullout Force Test Methodology and Effects of Polyurethane Block Density}

Another objective for this study was to ensure the methodology of suture anchor pullout was comparable to previous work for confidence in the setup and results. Forty pullout tests using two different density polyurethane blocks and two different suture anchors were effectively able to demonstrate this. The results of these pullout tests compared well against a previous pullout study by Nien.

From the results, the study verified that a $6.5 \mathrm{~mm}$ Smith and Nephew suture anchor pulled out of a polyurethane block at a similar force to a $1 / 4-20$ screw used in the study by Nien. These two anchors differed by $3 \%$ in pullout force when compared in $81 b$ density blocks. This gives confidence in the techniques and results of this study. 
A side takeaway from this study was the effects of polyurethane density on the pullout force. From the validation tests, the density of the block had a considerably larger influence on pullout force than did the anchor choice. This finding supports the idea that understanding the bone density and choosing the optimum insertion site is the crucial factor for surgeons to obtain the strongest suture anchor interface for healing. Common practice places suture anchors in the greater tuberosity, which has been found to have the highest density bone, but verification using image analysis and bone density readings should be done to confirm for each patient.

\subsection{Limitations and Strengths of the Study}

This study has certain known limitations. The first limitation is the use of noncadaver bones as the bed for the suture anchors. For this study, variability needed to be limited to best isolate the contributions of suture anchors, elevation angles, and bone densities. Cadaver bones are not homogenous and would add a large factor of variability into the data. Along the same lines, this study should been seen as a best-case scenario in terms of the pullout forces. The majority of patients who need rotator cuff surgery are elderly. The SAWBONES humeri used have higher and more consistent densities than these patients, so therefore realistic pullout forces will be lower.

Another limitation of the study is the assumption that the supraspinatus tendon is the only muscle contributor to humeral frontal elevation. This resultant one line of action is applicable because the most common type of rotator cuff injury is the tearing of the supraspinatus tendon whose primary movement is in the frontal plane. Also, since there will be contributions from the deltoid on the elevation, the amount of force placed on the anchor will be less. 
One strength of the study was the implementation of a design of experiments to better optimize the number of samples needed to describe the contributing effects of the suture anchor and elevation angle. Eighteen tests were sufficient to differentiate these two factors and effectively map their effects on the pullout force. Systematic DOEs are extremely useful when test materials are valuable and/or not readily available.

The design of experiments and statistical analysis was also able to create prediction estimates to create equations relating pullout force to elevation angle. All three equations had negative slopes indicating an increase in angle decreases the pullout force. These equations can be helpful to obtain factors of safety and limits to shoulder elevation for rehabilitation. If physical therapists know the angle to which failure is more likely to occur, or even the amount a force the suture anchor can withstand at a certain elevation angle, they can better tailor their exercises and optimize the healing. A limitation to these prediction equations is that they are suture anchor dependent. A solution to this would be to create a pullout force equation that will allow input of suture anchor dimensions as well as angles. 


\section{CHAPTER 6.0 CONCLUSIONS/RECOMMENDATIONS FOR FUTURE WORK}

\subsection{Conclusions}

This experimental study looked at the effects of frontal elevation angle of the humerus and suture anchor selection on pullout force. The results and analysis help with the understanding of the maximum forces that could be placed on suture anchors used in rotator cuff repair. The main conclusions to be drawn from this study are reported below:

1) The angle, anchor, and density all have a significant effect on the pullout force of suture anchors. The angle and anchor were shown to have relatively similar effects, and the bone density had a very large effect.

2) As the elevation of the humerus is increased in the frontal plane, the amount of force needed to pullout the suture anchor is decreased.

3) Larger suture anchors provide a higher pullout force than smaller sizes, but result in a more traumatic failure mode.

4) Higher density bones provide substantially higher pullout forces.

When dealing with a torn rotator cuff, the priority of the physician should be to first identify the optimum insertion site that offers the highest density bone for implantation. Once this has been found, a suitable suture anchor should be chosen based on its pullout strength.

\subsection{Recommendations for Future Work}

Future work can include the finer resolution of pullout force versus elevation angle. This study was able to provide a linear prediction equation for each suture anchor using three angle points and three suture anchors. The pullout force curve between these 
angles may not follow a linear path so the next study should analyze more elevation angles using only one type of suture anchor.

A subsequent study to this work should also be to model the suture anchor pullout using in vivo settings. This would include the use of cadaver bone, a saline environment, temperature at 37 degrees Celsius, and cyclic loading. This will give an understanding to the gap, if any, between these results and the results from factors seen in vivo.

Lastly, the larger goal for future work will be to create an equation that incorporates the variables of suture anchors, bone density, and other factors to create an equation that can be used to optimize suture anchor selection based on patient's anatomy. This would be a large undertaking and may need intermediate studies. 


\section{BIBLIOGRAPHY}

1. Ammon, Jennifer Tucker, Nyland, John, Chang, Haw Chong, Burden, Robert, Caborn, David N. M., Evaluation of BioCorkscrew and Bioknotless RC Suture Anchor Rotator Cuff Repair Fixation: An In Vivo Biomechanical Study Knee Surgery Sports Traumatol Arhrosc Vol. 15 No. 11 pp. 1375-1381 2007.

2. Azato, Flavia Namie, Yamasaki, Andre Toraso, Succomine, Fabio, Neto, Arnaldo Amado Ferriera, Filho, Americo Zoppi, Benegas, Eduardo, Pacheco, Alexandre Pagotto, Neto, Raul Bolliger and Pereira, Cesar Augusto Martins Traction Endurance Biomechanical Study of Metallic Suture Anchors at Different Insertion Angles Vol. 11 No. 1 pp. 25-31 2003.

3. Beleani, Massimiliano, Schrader, Stephan, Veronesi, Carlo Andrea, Rotini, Roberto, Giardino, Roberto and Toni, Aldo Surgical Repair of the Rotator Cuff: A Biomechanical Evaluation of Different Tendon Grasping and Bone Suture Fixation Techniques Clinical Biomechanics Vol. 18 No. 8 pp. 721-729 2003.

4. Bynum, C. Kelly, Lee, Steven, Mahar, Andrew, Tasto, James and Pedowitz, Robert, Failure Mode of Suture Anchors as a Function of Insertion Depth The American Journal of Sports Medicine Vol. 33 No. 7 pp. 1030-1034 2005. 
5. Chapman, J. R., Harrington, R. M., Lee, K. M., Anderson, P. A., Tencer, A. F. and Kowalski, D., Factors Affecting the Pullout Strength of Cancellous Bone Screws Journal of Biomechanical Engineering Vol. 118 No. 3 pp. 391-398 1996.

6. Gray, Henry. Anatomy of the Human Body. Philadelphia: Lea \& Febiger, 1918.

7. Harryman II, Douglas T., Hettrich, Carolyn M., Smith, Kevin L., Campbell, Barry, Sidles, John A. and Matsen III, Frederick A. A Prospective Multipractice Investigation of Patients with Full-Thickness Rotator Cuff Tears The Journal of Bone and Joint Surgery Vol. 85-A No. 42003.

8. Harryman II, Douglas T., Mack, Laurence A., Wang, Keith Y., Jackins, Sarah E. R.P.T., Richardson, Michael L. and Matsen III, Frederick A., Repairs of the Rotator Cuff The Journal of Bone and Joint Surgery Vol. 73-A No. 7 pp. 982-989 1991.

9. Hawkins, Richard J., Misamore, Gary W. and Hobeika, Paul E., Surgery for FullThickness Rotator-Cuff Tears The Journal of Bone and Joint Surgery Vol. 67-A No. 9 pp. 1349-1355 1985.

10. Hsu, Ching-Chi, Chao, Ching-Kong, Wang, Jaw-Lin, Hou, Sheng-Mou, Tsai, Ying-Tsung and Lin, Jinn Increase of Pullout Strength of Spinal Pedicle Screws 
With Conical Core: Biomechanical Tests and Finite Element Analyses Journal of Orthopaedic Research Vol. 23 pp. 788-794 2005.

11. Iannotti, JP, Full-Thickness Rotator Cuff Tears: Factors Affecting Surgical Outcome Journal of the American Academy of Orthopaedic Surgeons Vol. 2 pp. 87-95 1994.

12. Illyés, Árpád, Kiss, Rita M., Shoulder Joint Kinematics During Elevation Measured by Ultrasound-Based Measuring System Journal of Electromyography and Kinesiology Vol. 17 No. 3 pp. 355-364 2006.

13. Itoi, Eiji, Berglund, Lawrence J., Grabowski, John J., Schultz, Fredrick M., Growney, Eric S., Morrey, Bernard F. and An, Kai-Nan, Tensile Properties of the Supraspinatus Tendon The Journal of Bone and Joint Surgery Vol. 13 No. 4 pp. 578-584 1995.

14. Liu, J., Hughes, R. E., Smutz, W. P., Niebur, G and Kan-An, K Roles of Deltoid and Rotator Cuff Muscles in Shoulder Elevation Clinical Biomechanics Vol. 12 No. 1 pp. 32-38 1997.

15. Matsen III, Frederick A. Rotator-Cuff Failure The New England Journal of Medicine Vol. 358 pp. 2138-2147 2008. 
16. Matsen III, Frederick A., "Repair of Rotator Cuff Tears: Surgery for Shoulders With Torn Rotator Cuff Tendons Can Lessen Shoulder Pain and Improve Function Without Acromioplasty." UW Medicine: Orthopaedics and Sports Medicine. December 23, 2009. January 2010.

$<$ http://www.orthop.washington.edu/rotatorcuff $>$

17. McCallister, Wren V., Parsons, I. Moby, Titelman, Robert M. and Matsen III, Frederick A. Open Rotator Cuff Repair without Acromioplasty The Journal of Bone and Joint Surgery Vol. 87-A No. 62005.

18. McFarland, Edward G., Park, Hyung Bin, Keyurapan, Ekavit, Gill, Harpreet S. and Selhi, Harpal S, Suture Anchors and Tacks for Shoulder Surgery, Part 1 The American Journal of Sports Medicine Vol. 33 No. 12 pp. 1918-1923 2005.

19. Nien, Yu-Hsun, Kalidindi, Surya R. and Siegler, Sorin Fixation Strength of Swellable Bone Anchors in Low-Density Polyurethane Foam The Journal of Biomedical Materials Research Vol. 58 No. 2 pp. 137-146.

20. Ottis, James C., Jiang, Ching-Chuan, Wickiewicz, Thomas L., Peterson, Margaret G. E., Warren, Russel F. and Santer, Thomas J., Changes in the Moment Arms of the Rotator Cuff and Deltoid Muscles with Abduction and Rotation The Journal of Bone and Joint Surgery Vol. 76-A No. 5 pp. 667-676 1994. 
21. Park, Hyung Bin, Keyurapan, Ekavit, Gill, Harpreet S., Selhi, Harpal S. and McFarland, Edward G. Suture Anchors and Tacks for Shoulder Surgery, Part II The American Journal of Sports Medicine Vol. 34 No. 1 pp. 136-144 2006.

22. Poppen, Norman K. and Walker, Peter S., Normal and Abnormal Motion of the Shoulder The Journal of Bone and Joint Surgery Vol. 58-A No. 2 pp. 195-201 1976.

23. "Rotator cuff injuries." Orthopaedic Specialists. January 6, 2010. February 2010. $<\mathrm{http} / / /$ www.orthspec.com/rotator_cuff_injuries.htm>

24. Rupp, Stefan, Georg, Thomas, Gauss, Christian, Kohn, Dieter and Seil, Romain, Fatigue Testing of Suture Anchors The American Journal of Sports Medicine Vol. 30 No. 2 pp. 239-247 2002.

25. Scibek, Jason S., Carpenter, James E. and Hughes, Richard E. Rotator Cuff Tear Pain and Tear Size and Scapulohumeral Rhythm Journal of Athletic Training Vol. 44 No. 2 pp. 148-159 2009.

26. Slaney, Suzanne L., "Arthoscopic shoulder surgery for the treatment of rotator cuff tears: why, when and how it is done." UW Medicine Orthopaedics and Sports Medicine. December 31, 2009. February, 2010. $<$ http://www.orthop.washington.edu/arthosurgrotacufftears > 
27. "Suture Anchors" Shoulderdoc. N.d. January, 2010.

$<$ http://shoulderdoc.co.uk/article.asp?section=468\#staticlink $>$

28. Tingart, Markus J., Apreleva, Maria, Zurakowski, David and Warner, Jon J.P., Pullout Strength of Sutute Anchors Used in Rotator Cuff Repair The Journal of Bone and Joint Surgery Vol. 85-A No. 11 pp. 2190-2198 2003.

29. Waltrip, Robert L., Zheng, Nigel, Dugas, Jeffrey R. and Andrews, James R., Rotator Cuff Repair: A Biomechanical Comparison of Three Techniques The American Journal of Sports Medicine Vol. 31 No. 4 pp. $493-4972003$.

30. Wuelker, N., Wirth, C. J., Plitz, W. and Roetman, B., A Dynamic Shoulder Model: Reliability Testing and Muscle Force Study Journal of Biomechanics Vol. 28 No. 5 pp. 489-499 1995. 
APPENDIX A: DOE Randomized Run Order 


\begin{tabular}{|c|c|}
\hline Anchor & Angle \\
\hline 3 & 3 \\
\hline 3 & 2 \\
\hline 2 & 3 \\
\hline 1 & 3 \\
\hline 2 & 2 \\
\hline 2 & 1 \\
\hline 2 & 1 \\
\hline 1 & 1 \\
\hline 3 & 2 \\
\hline 1 & 2 \\
\hline 2 & 3 \\
\hline 1 & 2 \\
\hline 1 & 3 \\
\hline 2 & 2 \\
\hline 3 & 1 \\
\hline 3 & 3 \\
\hline 3 & 1 \\
\hline 1 & 1 \\
\hline
\end{tabular}


APPENDIX B: Graphical Summaries of Pullout Tests 

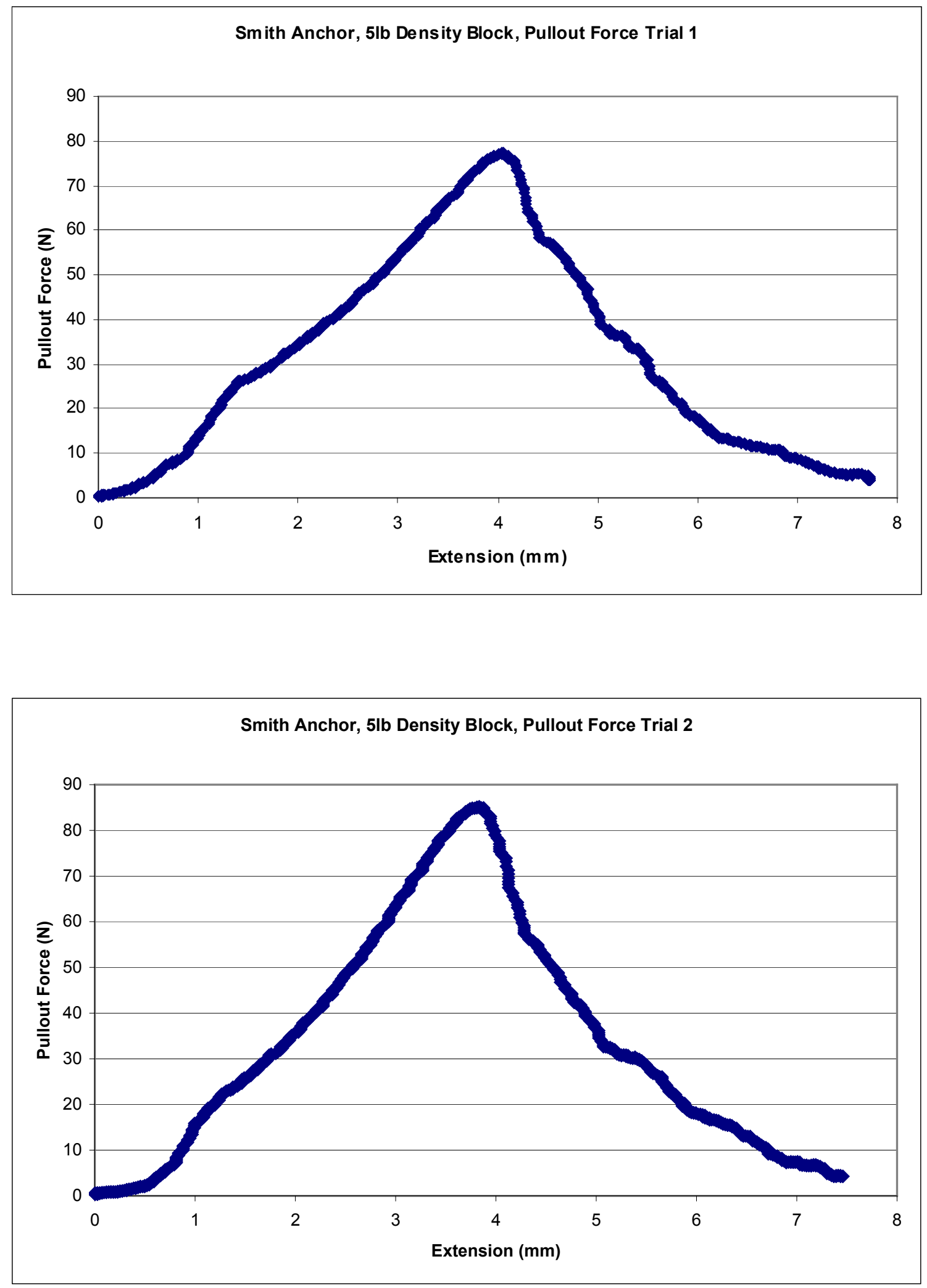

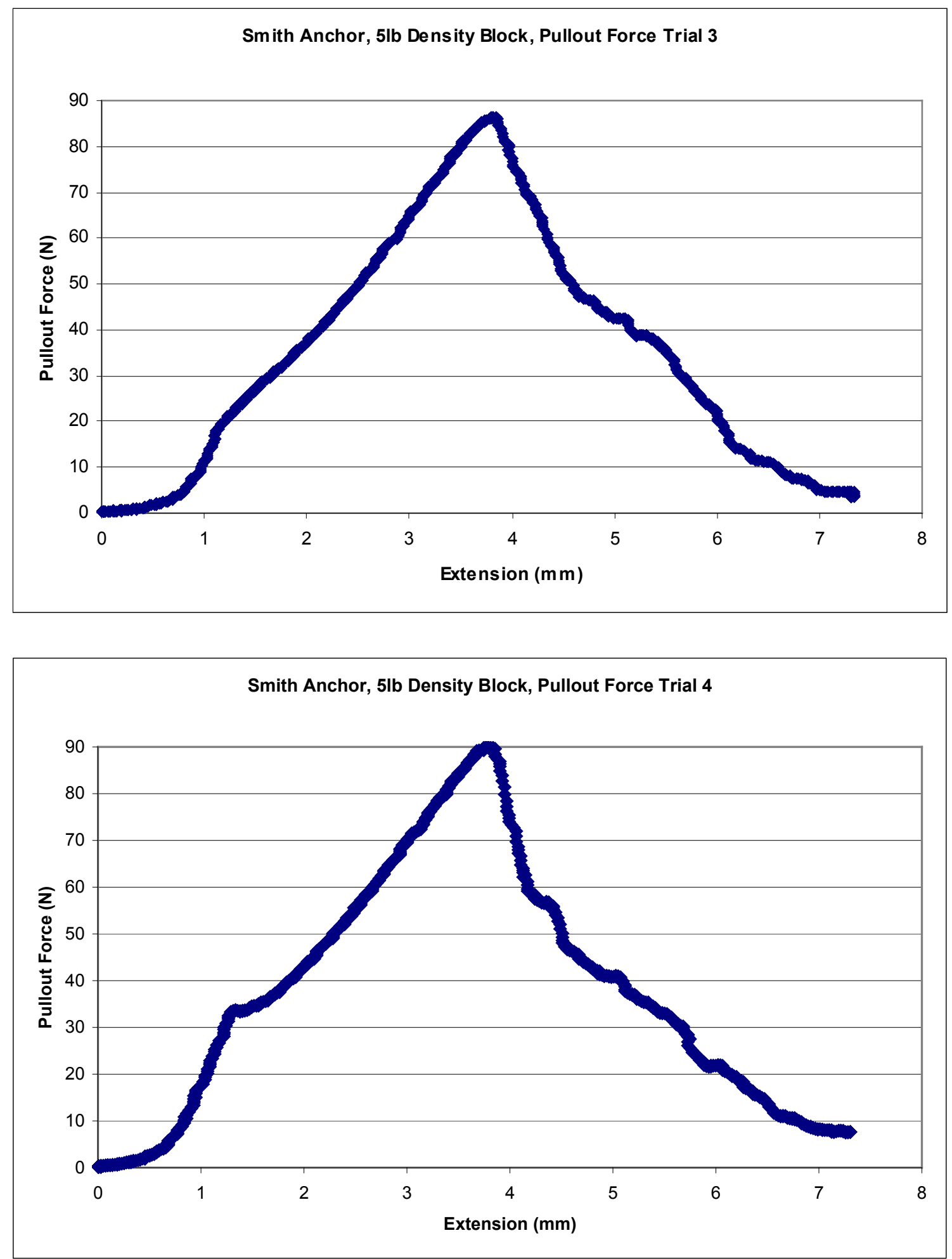

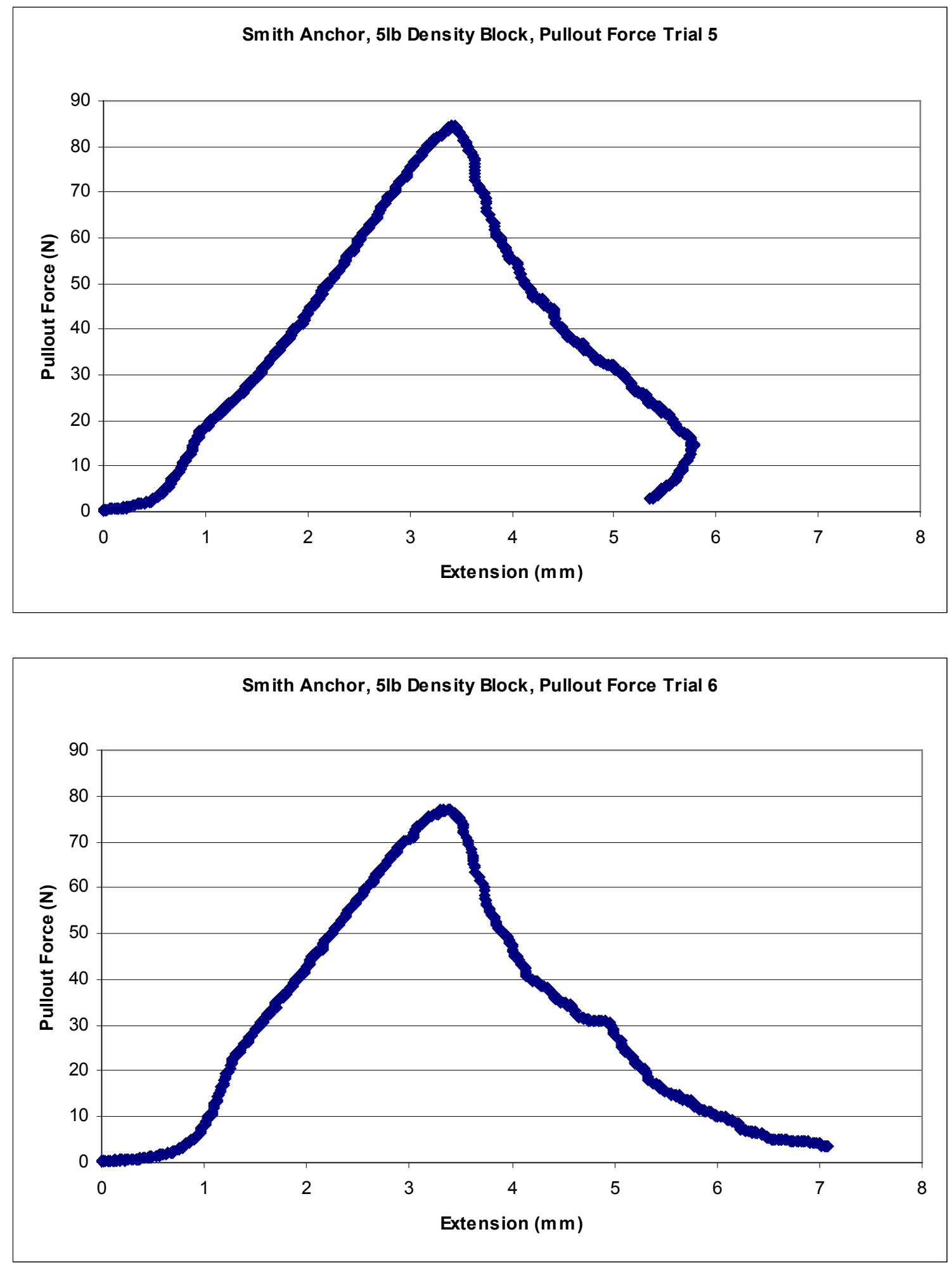

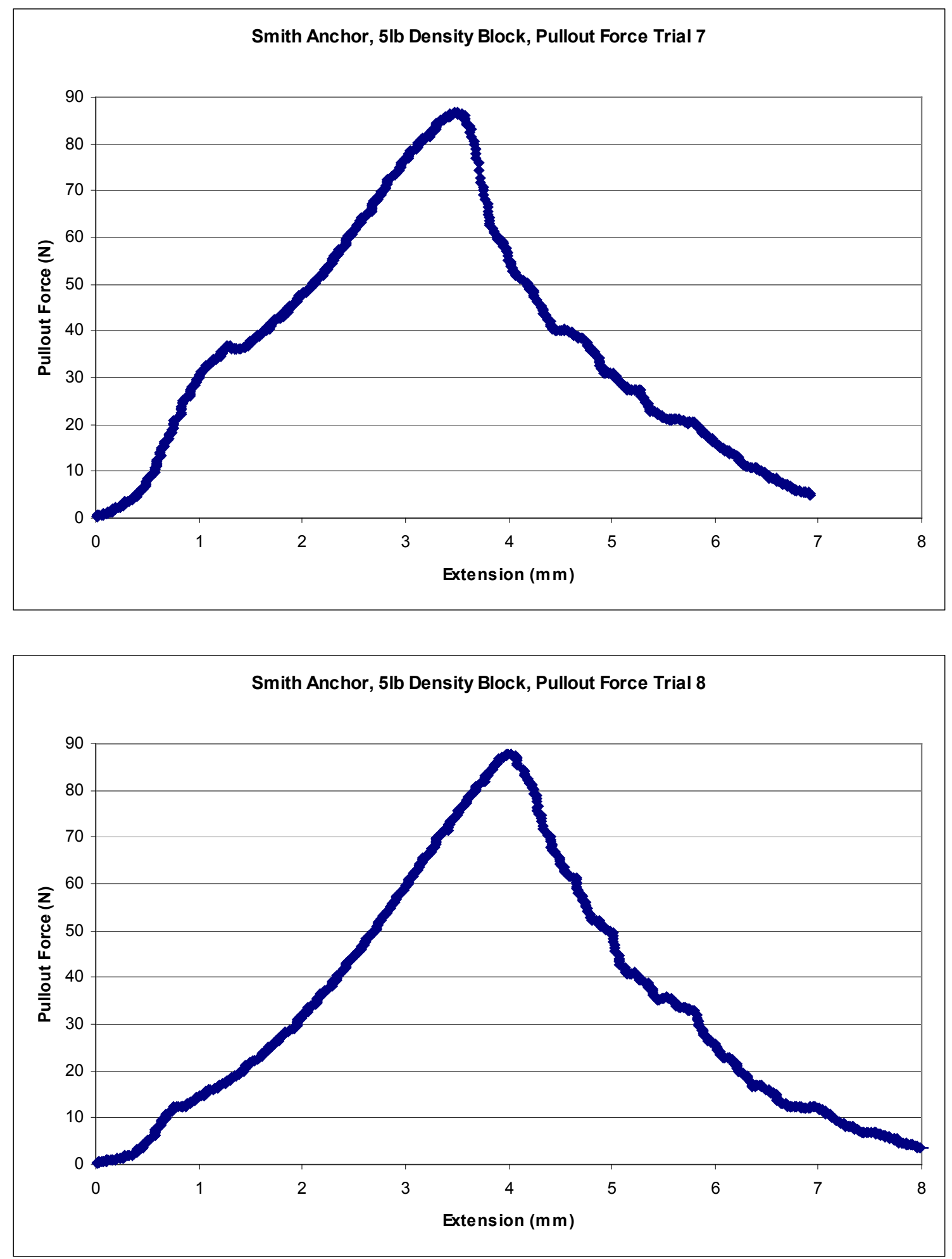

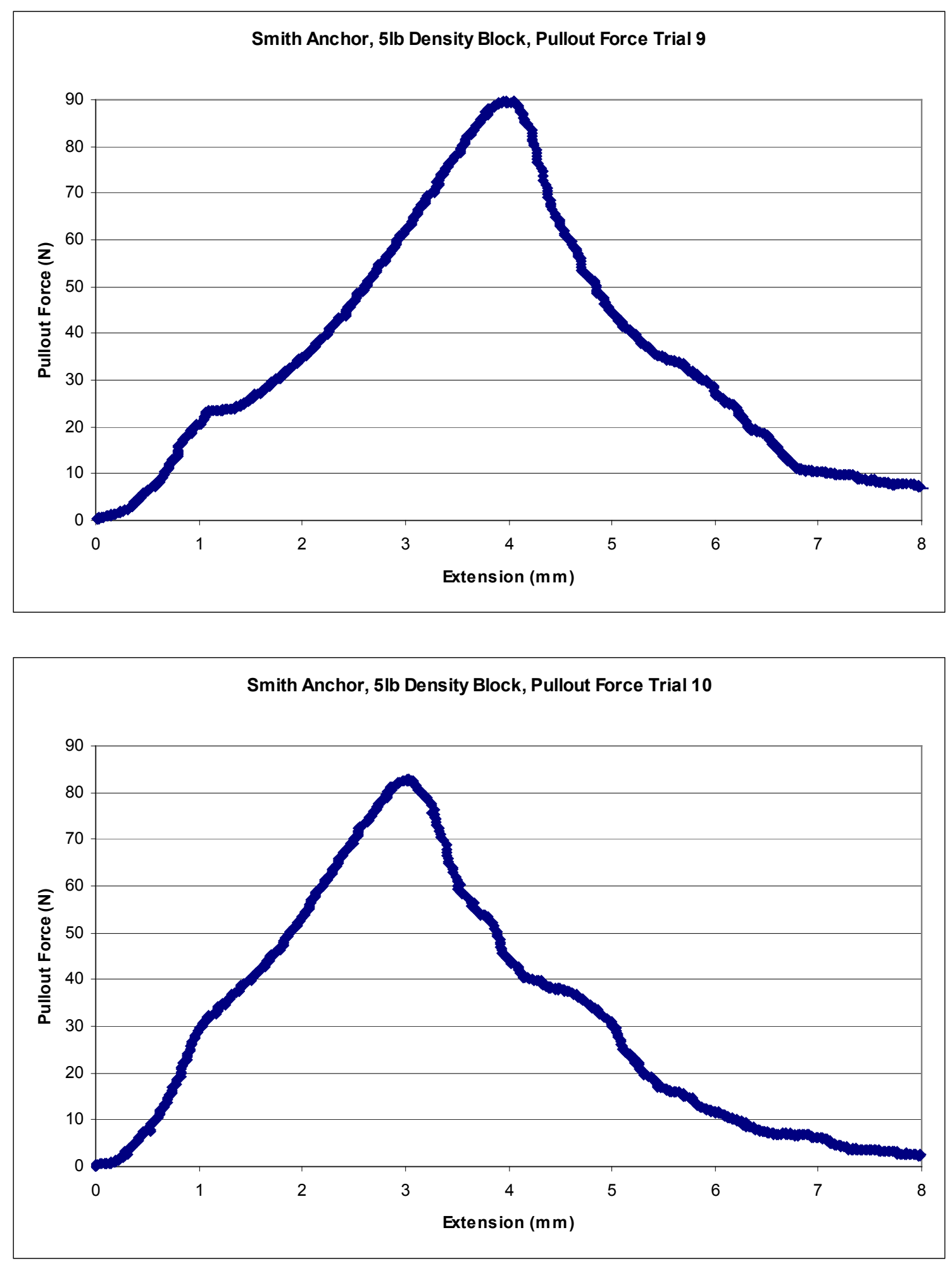

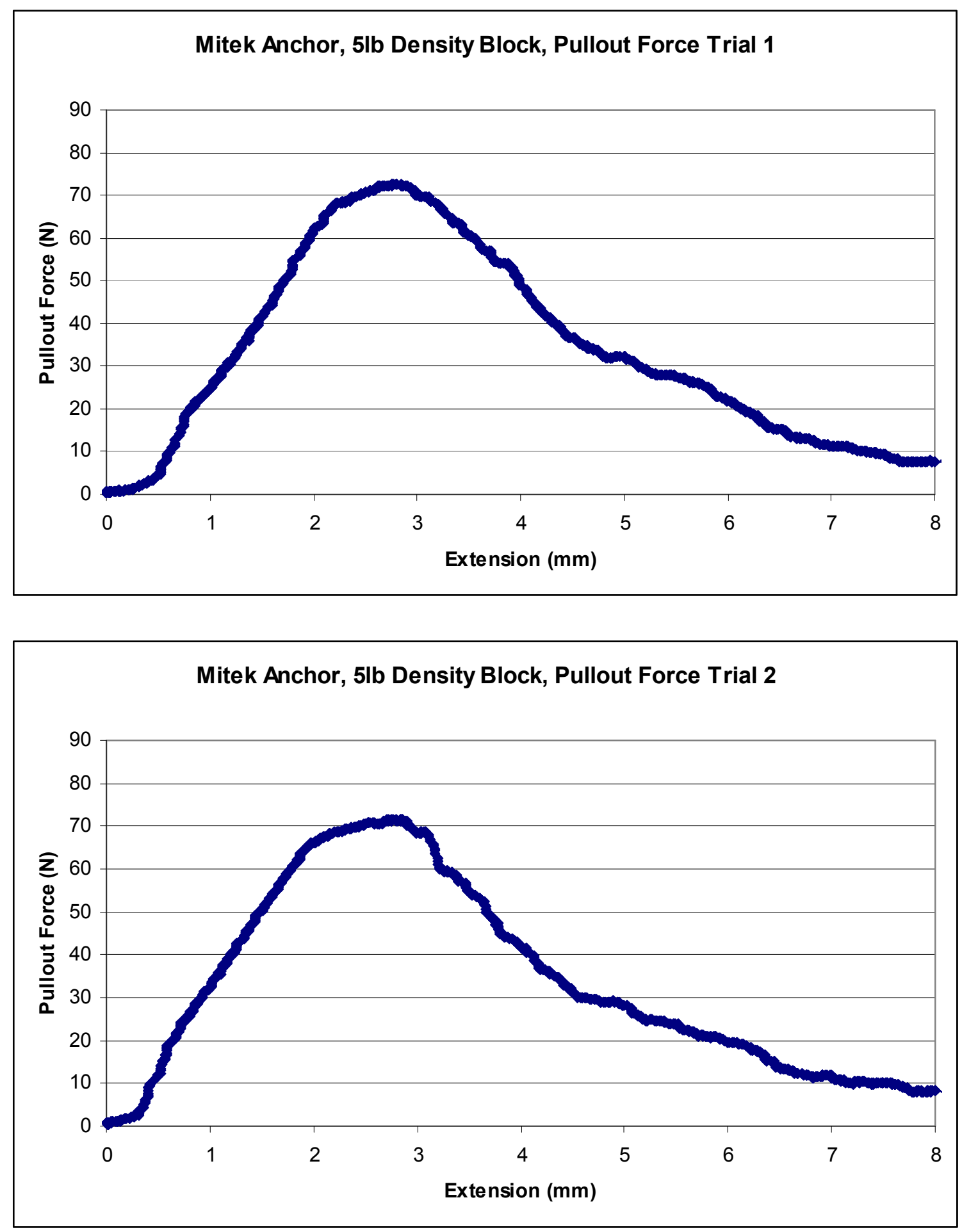

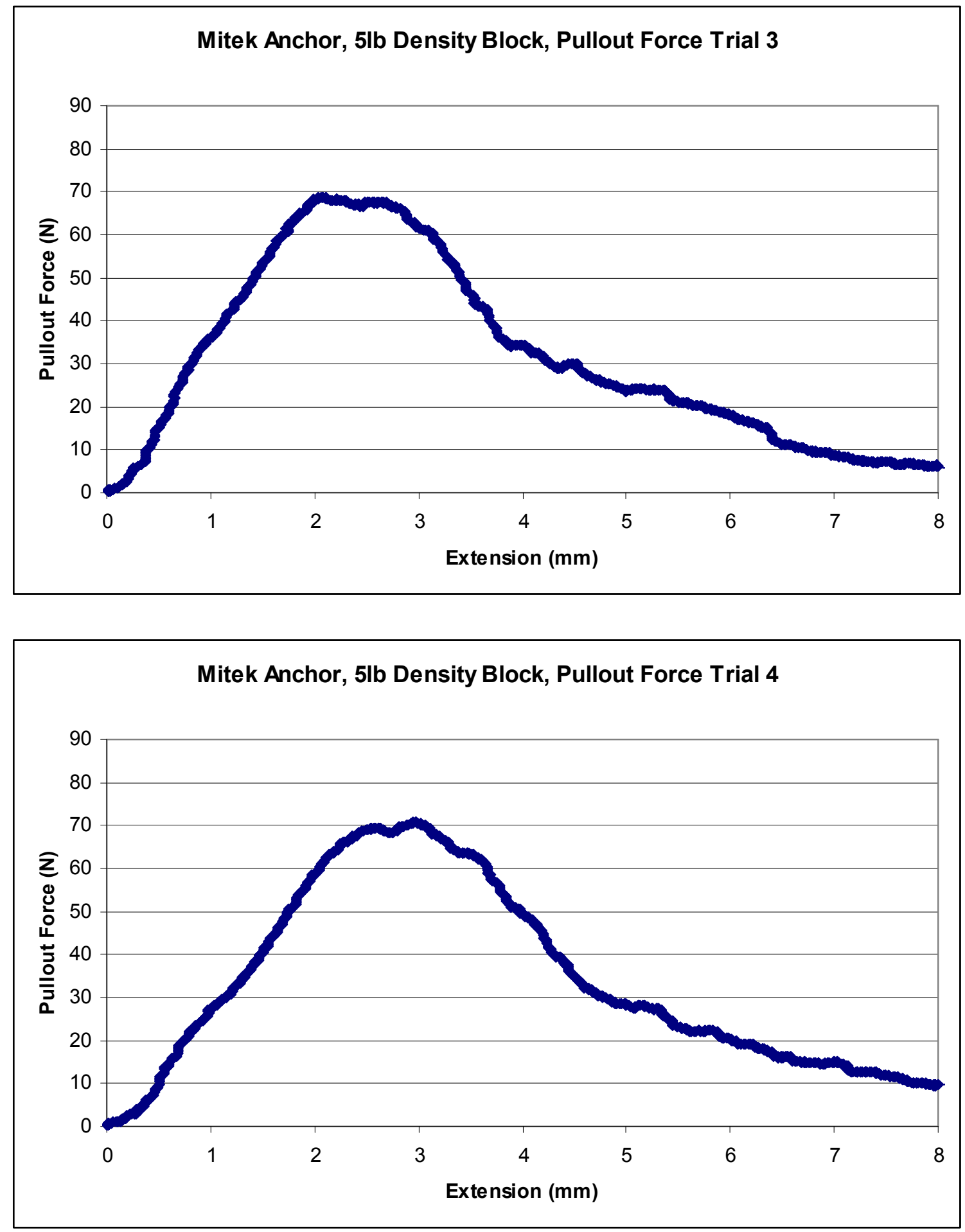

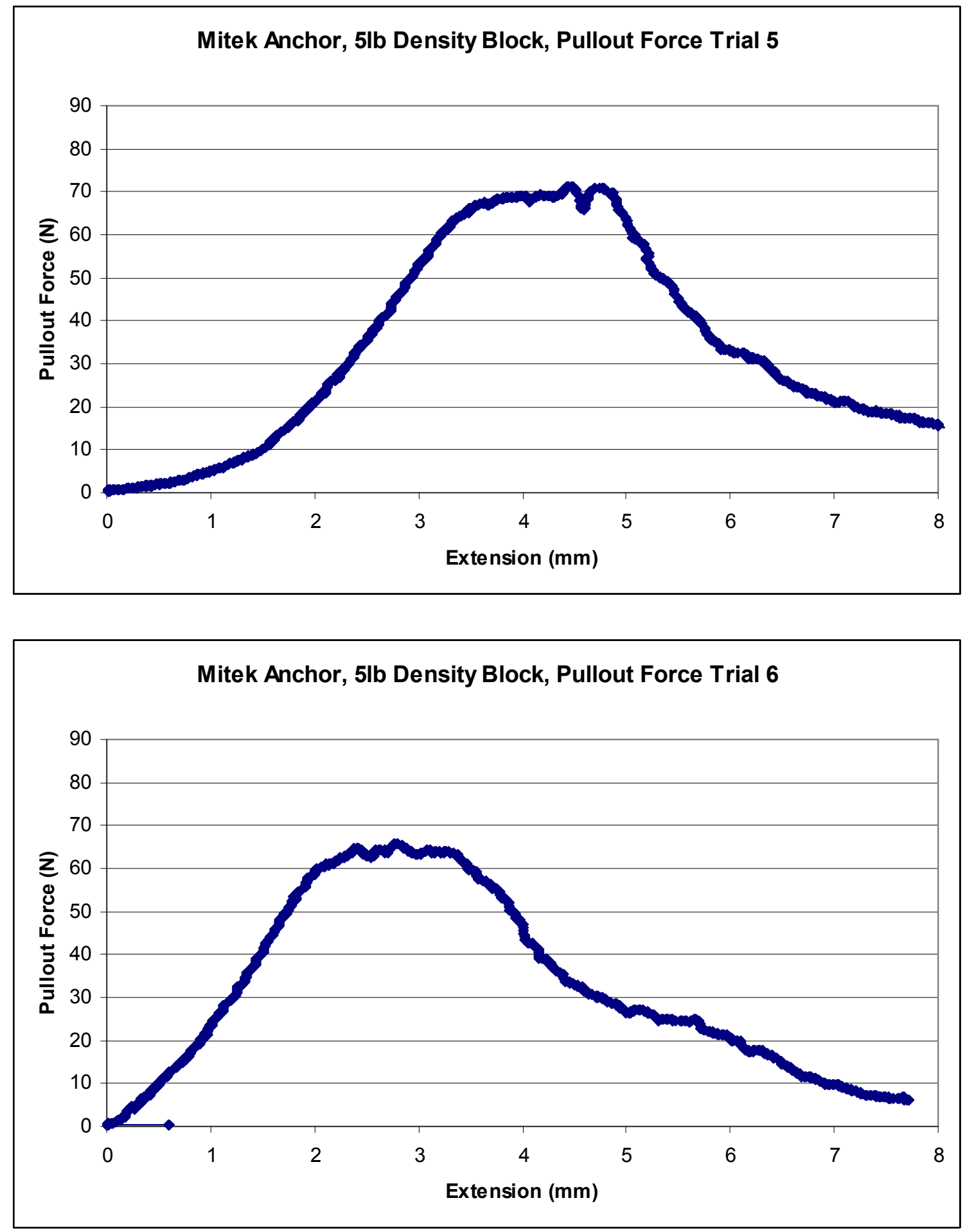

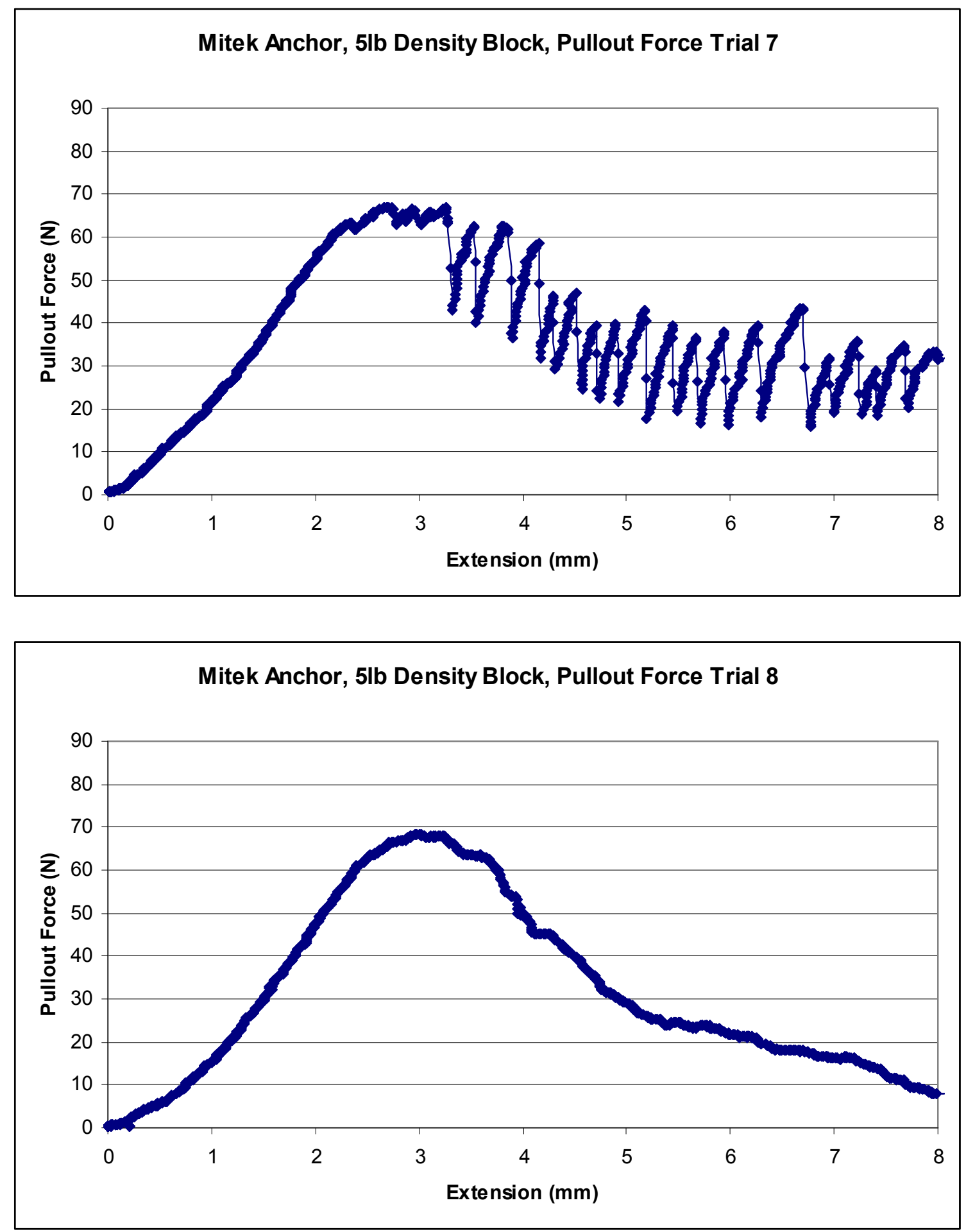

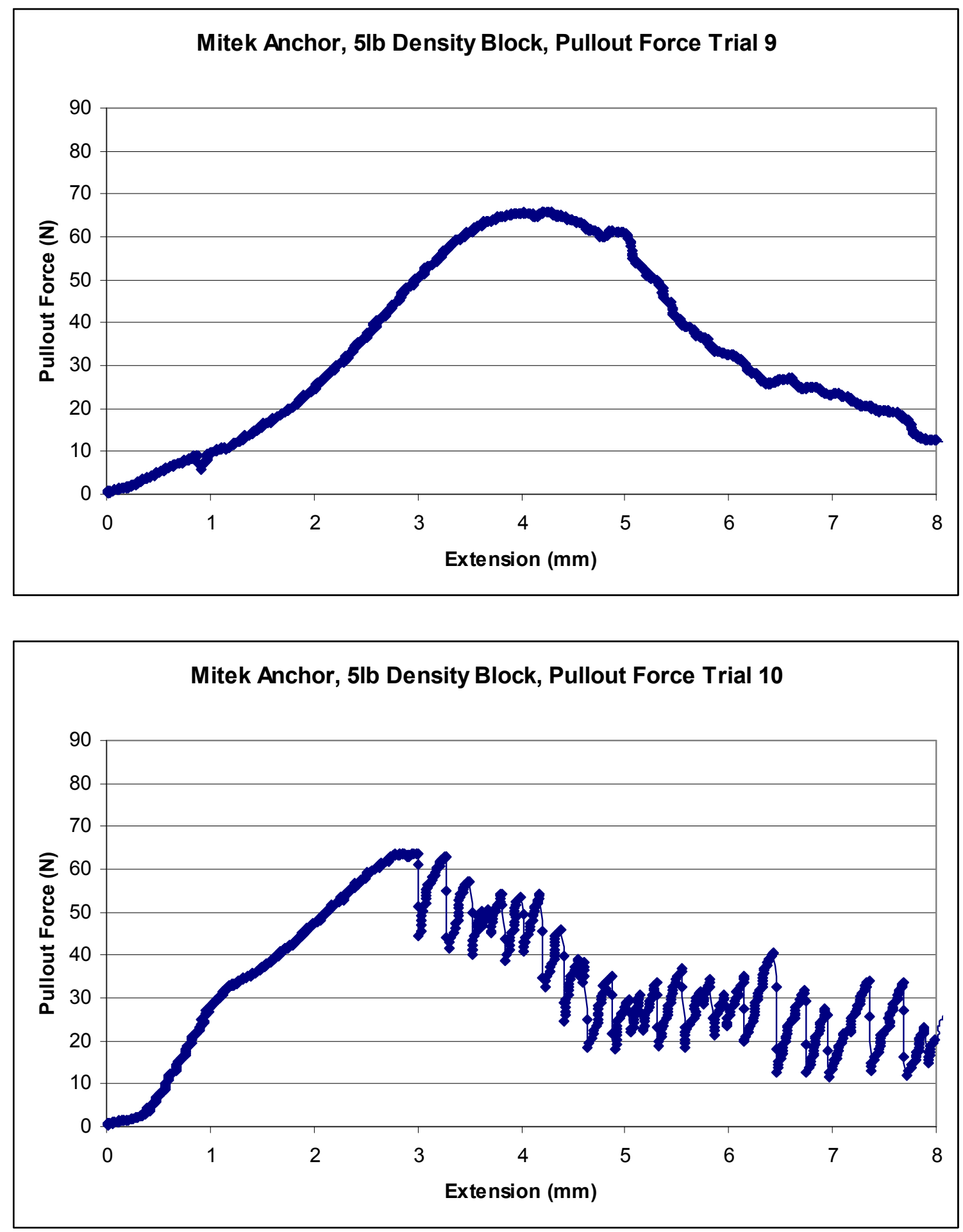

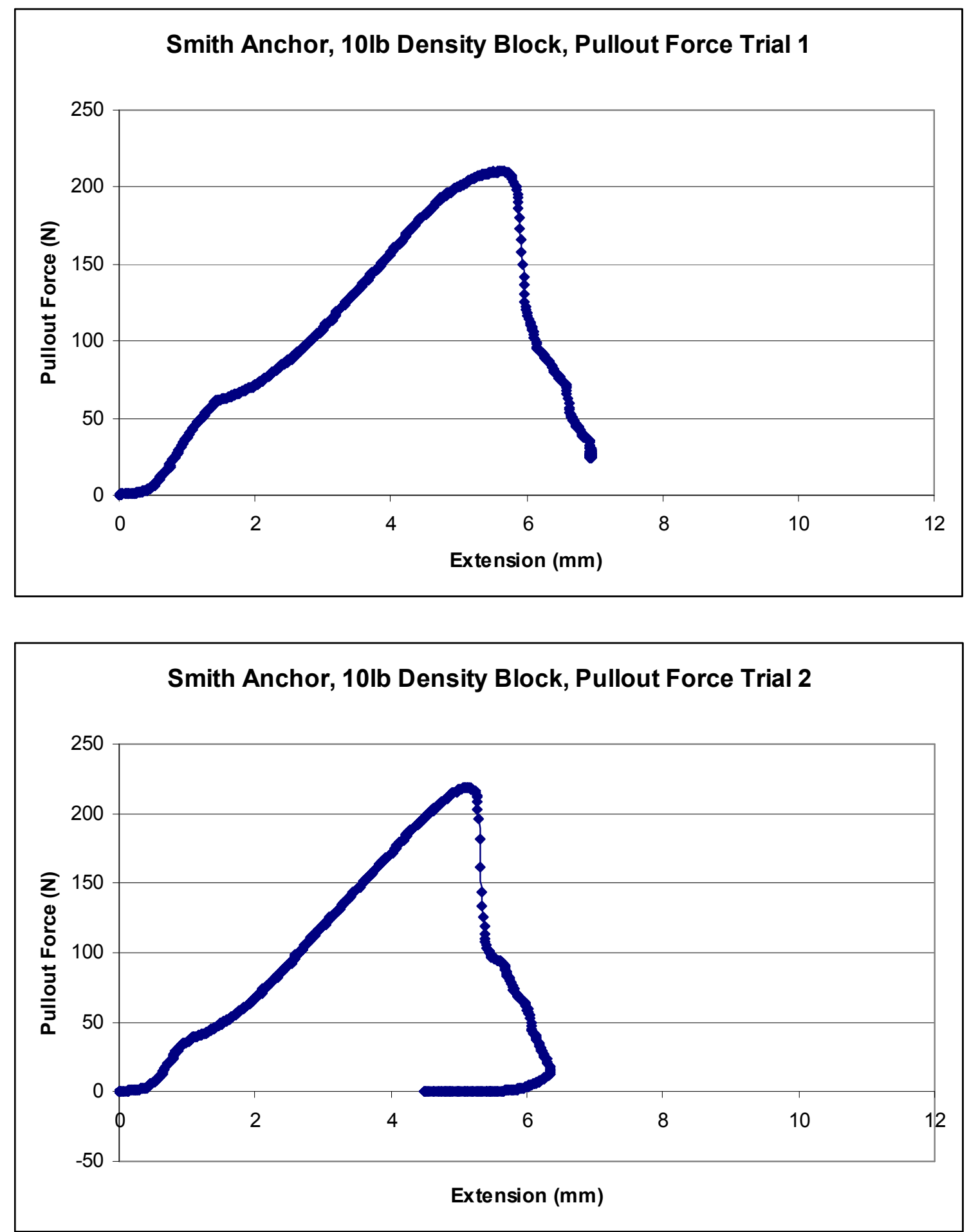

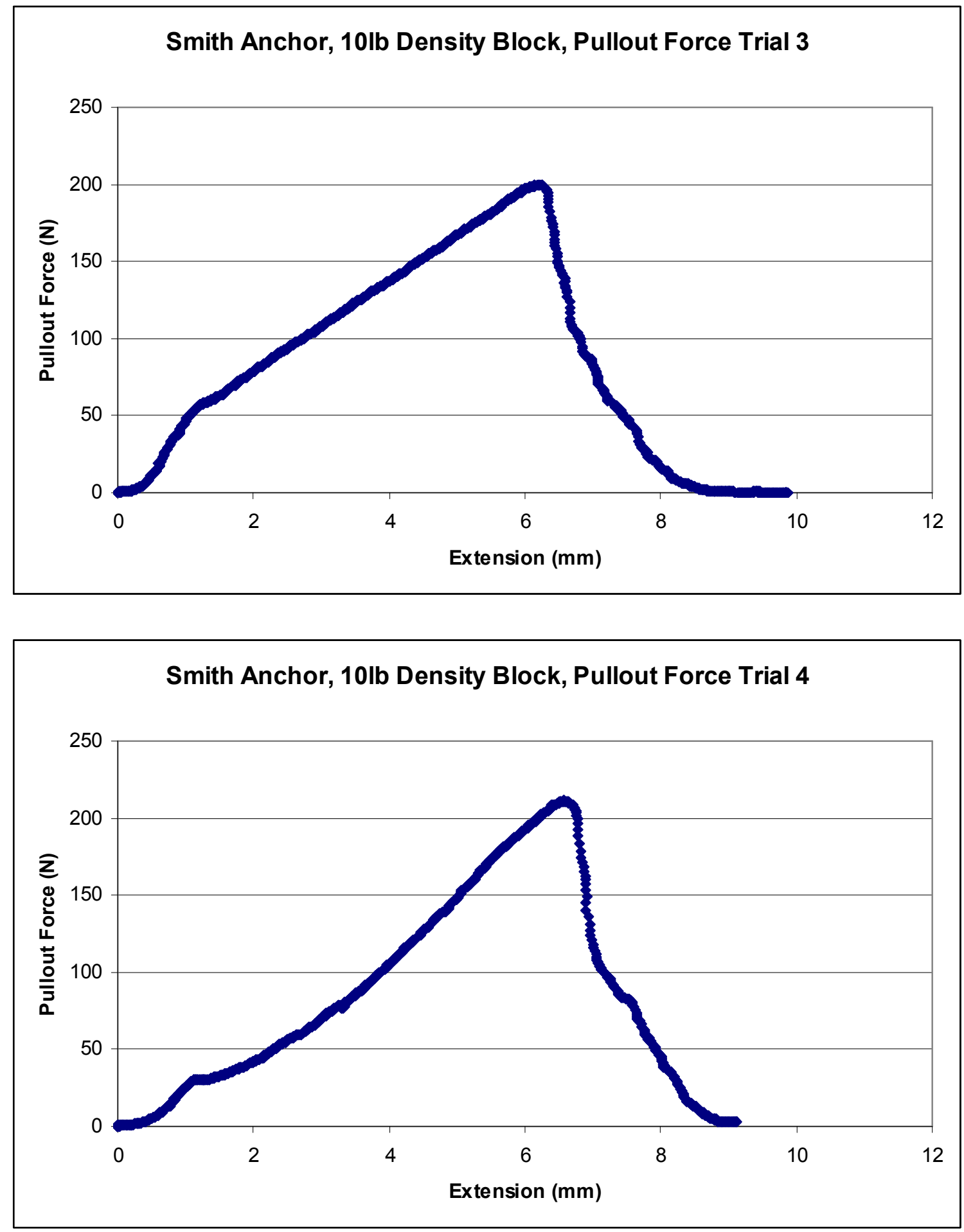

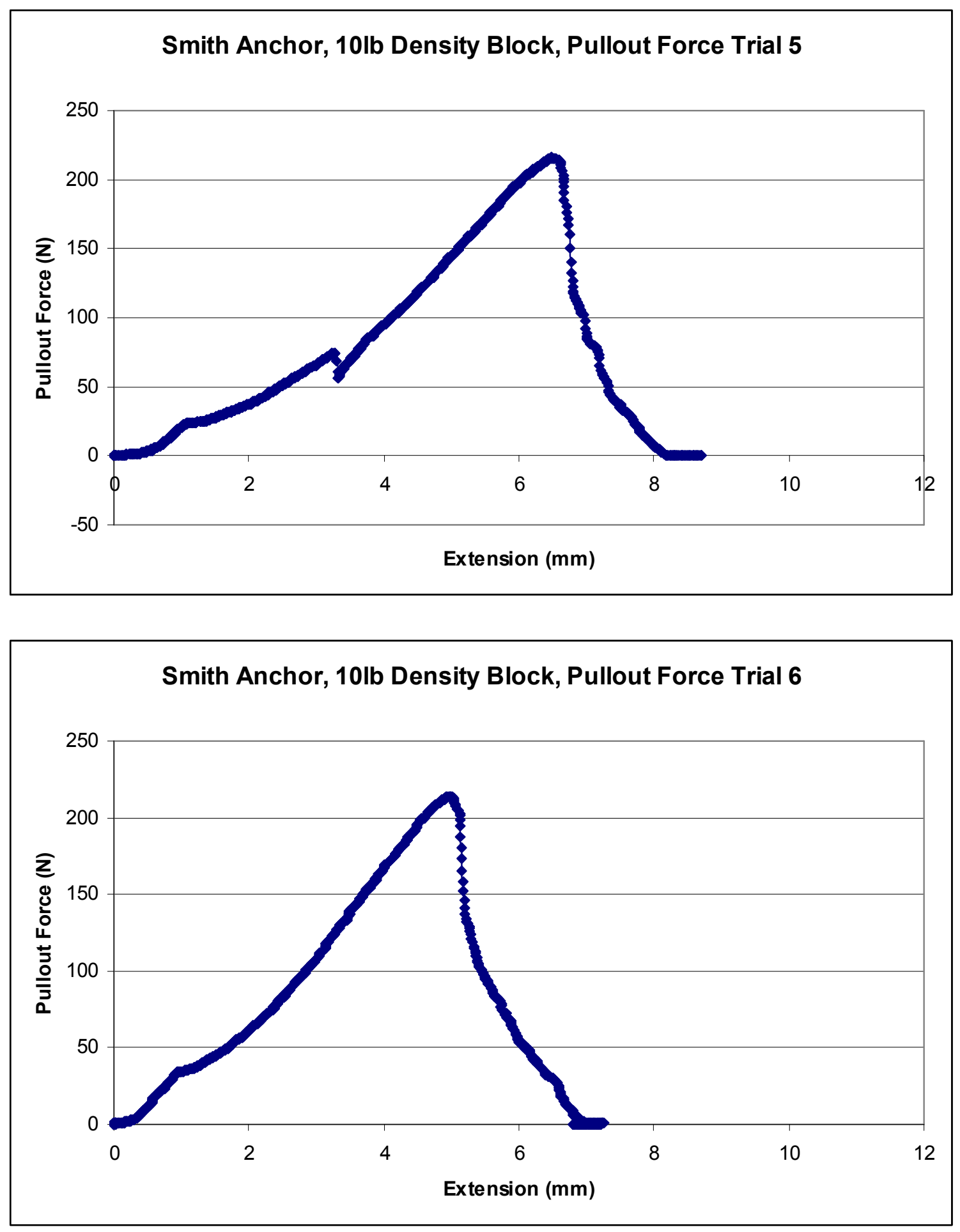

59 

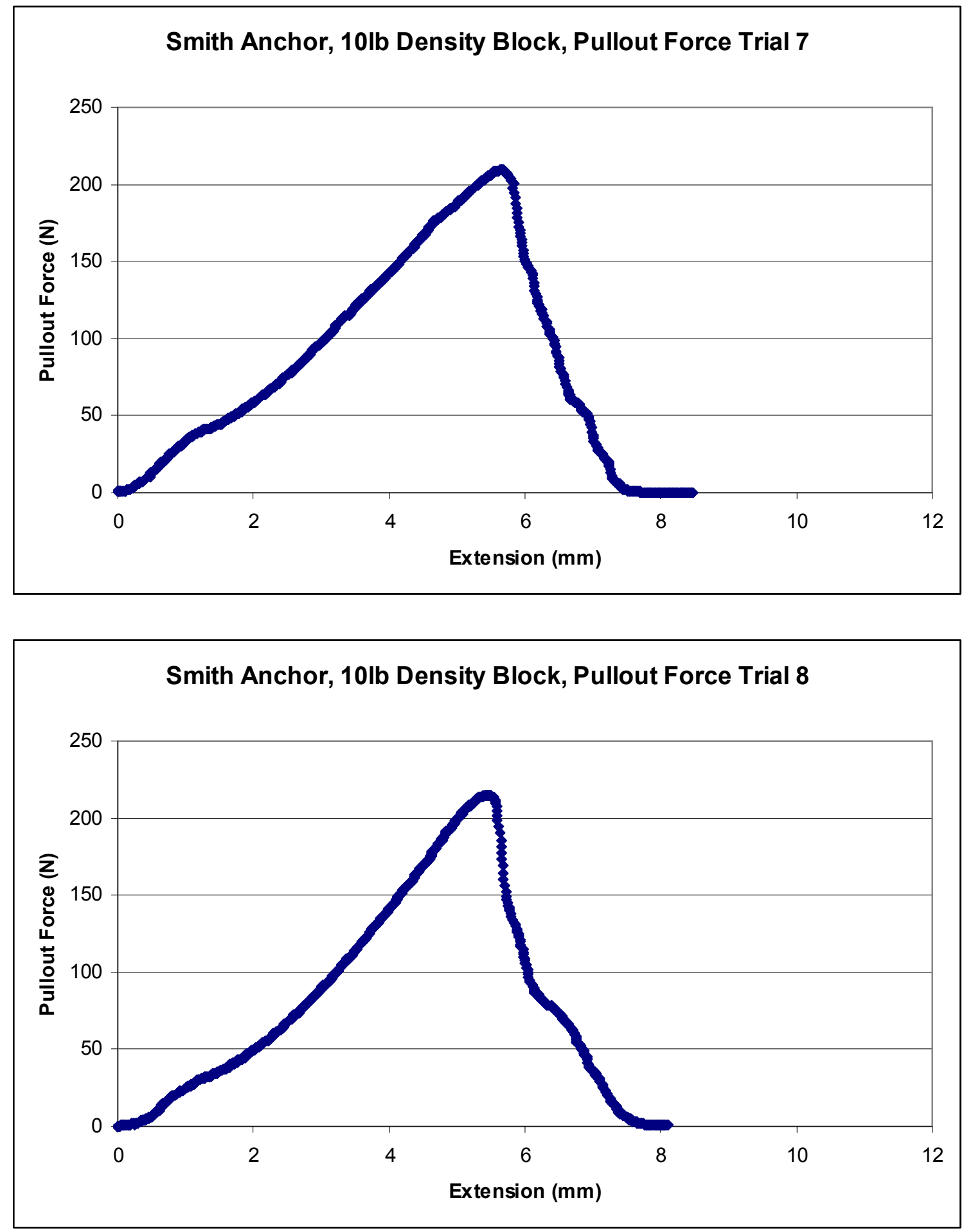

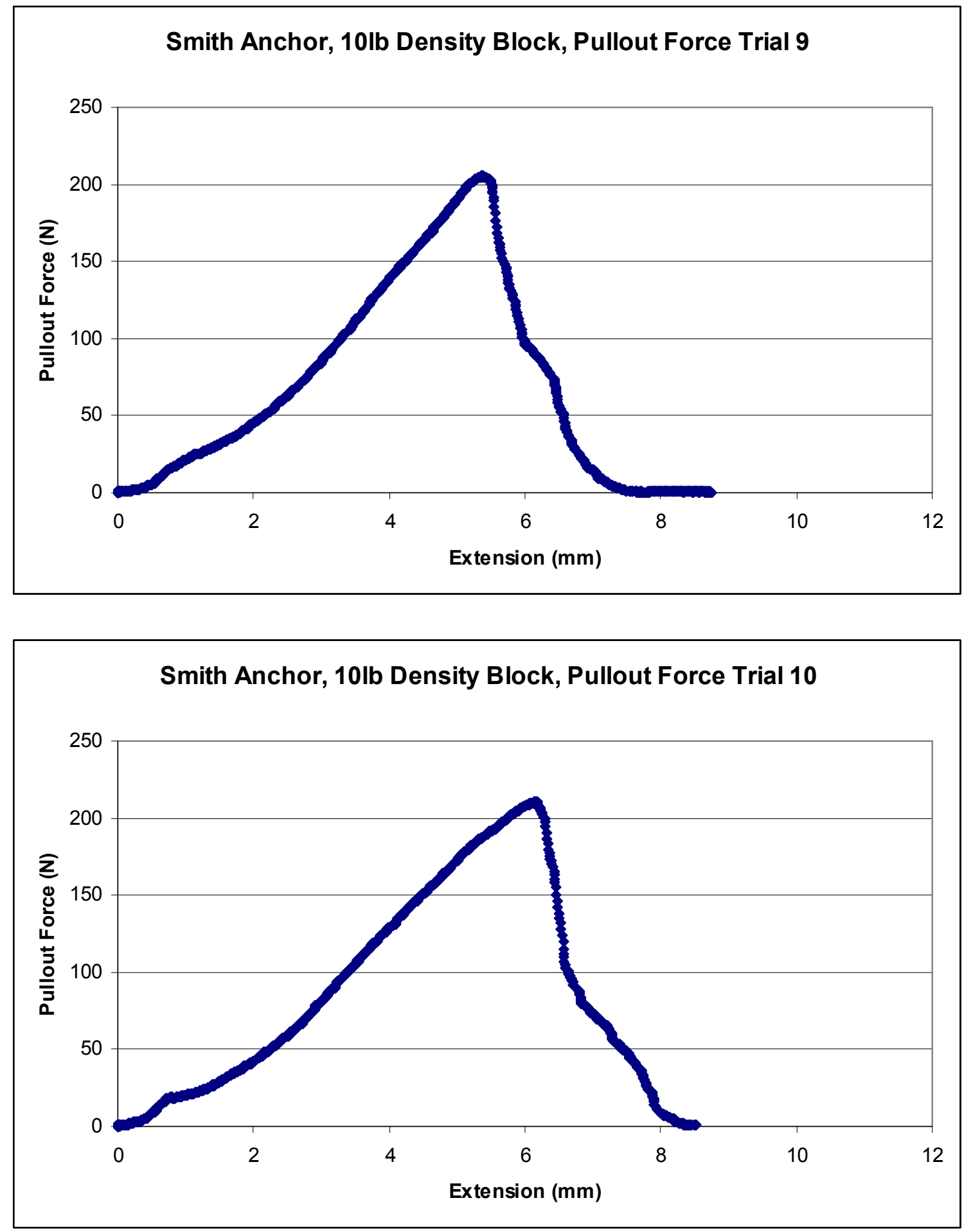

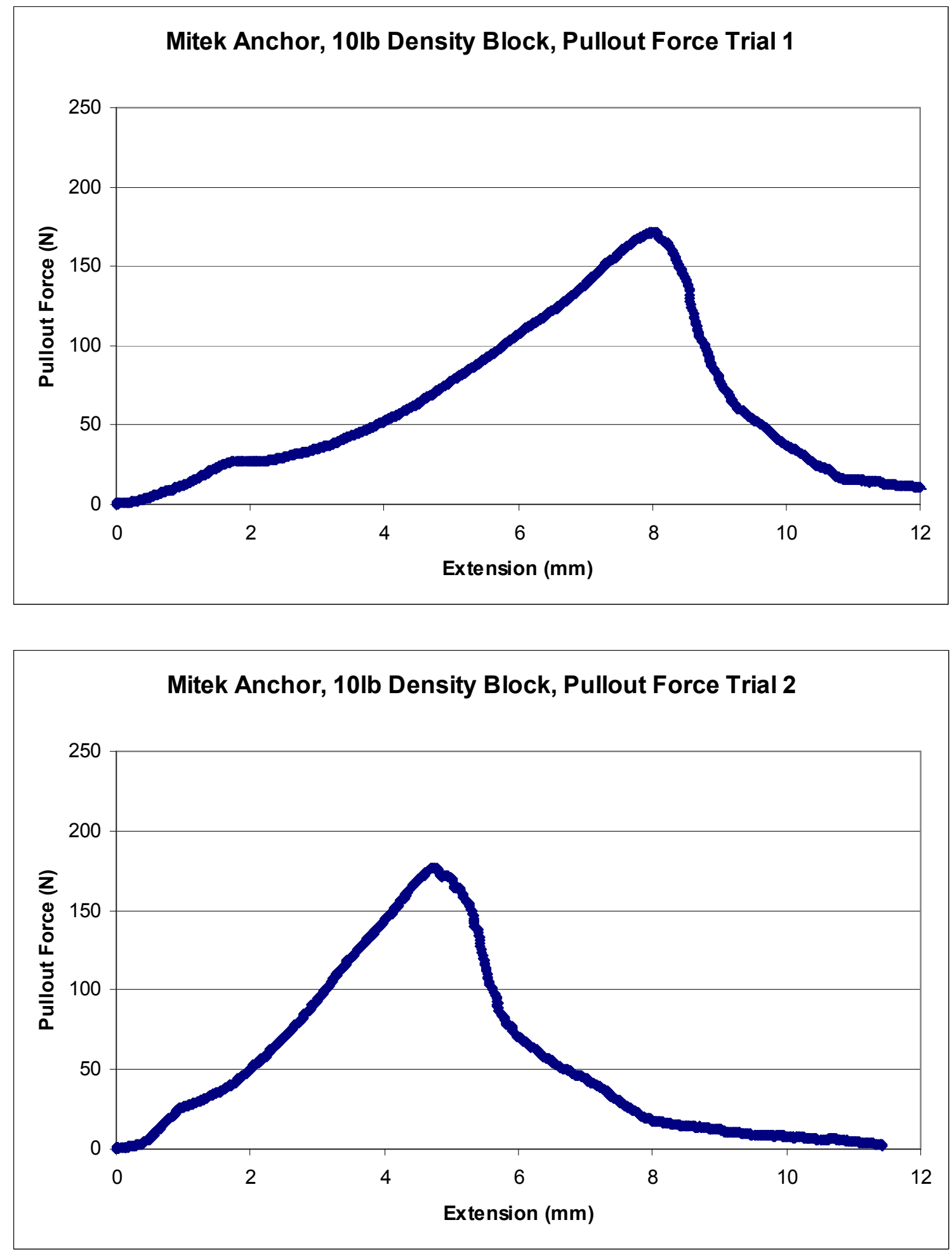

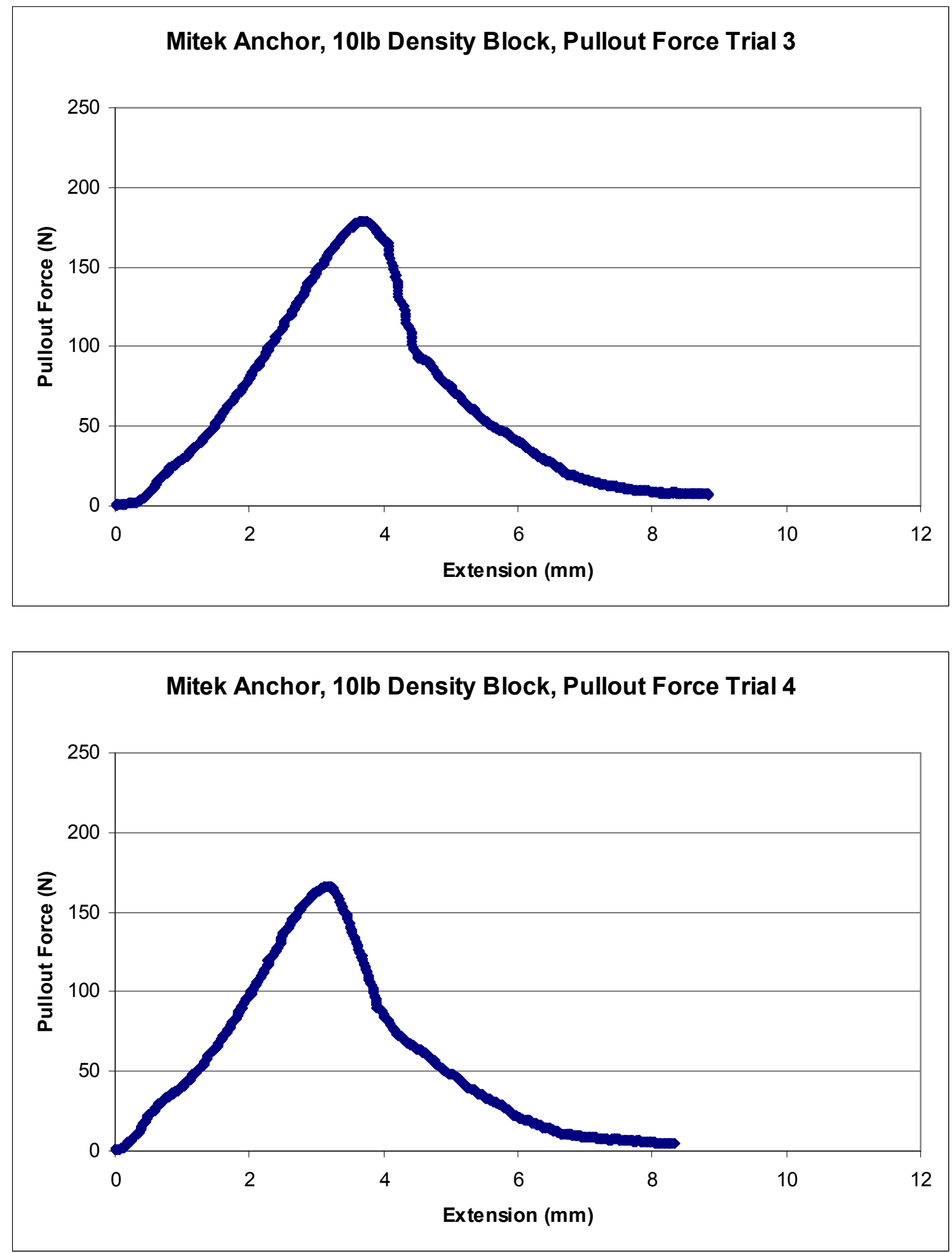

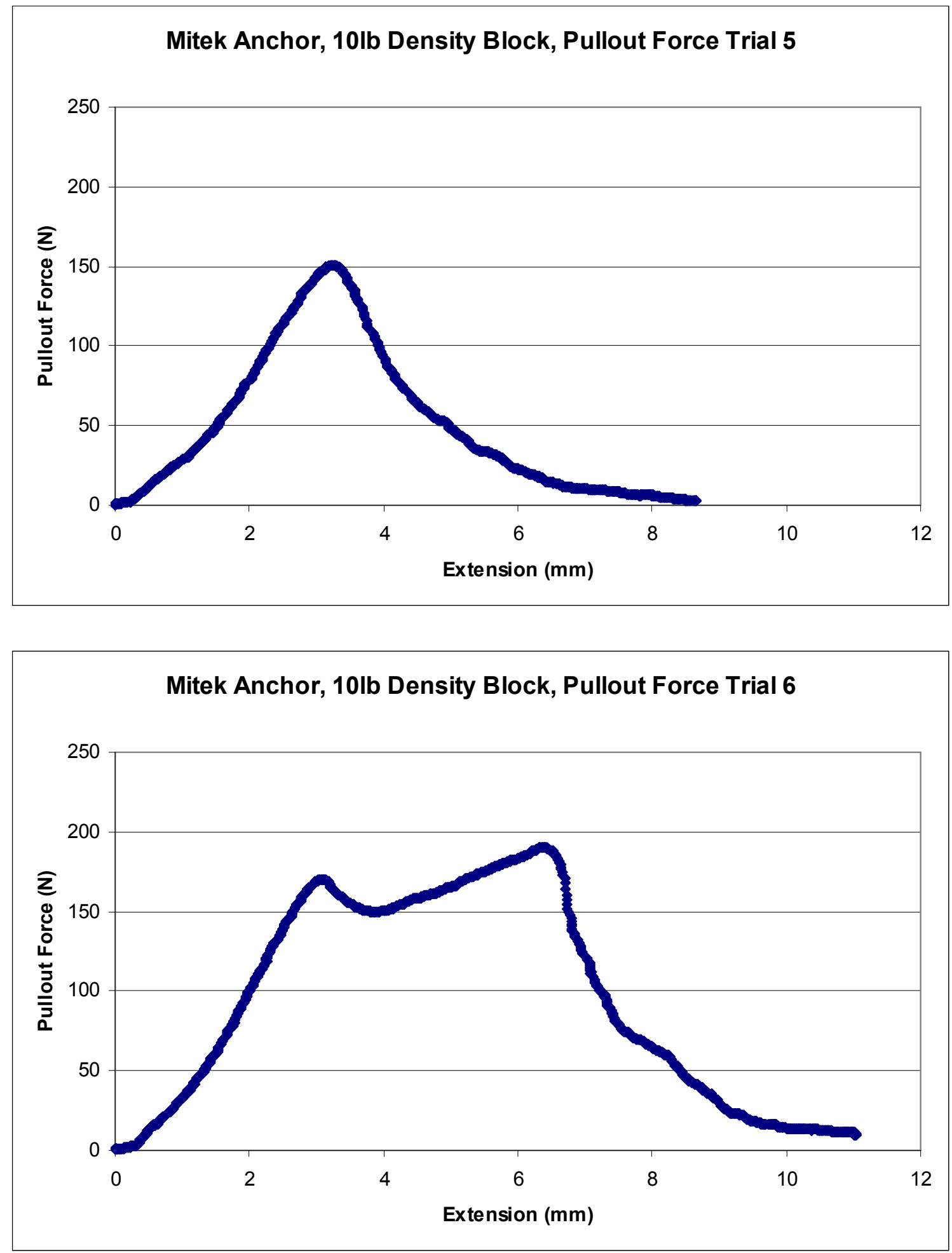

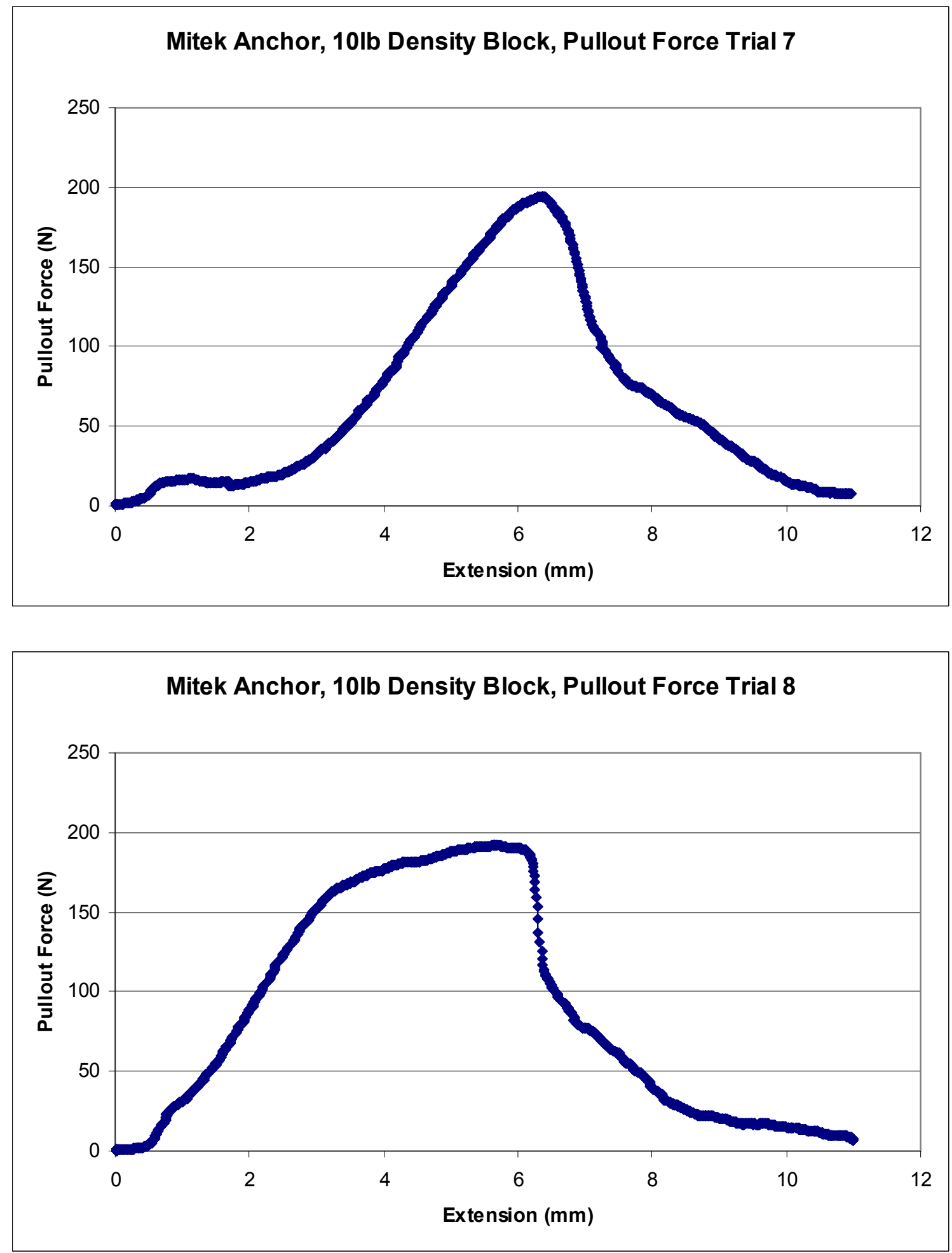

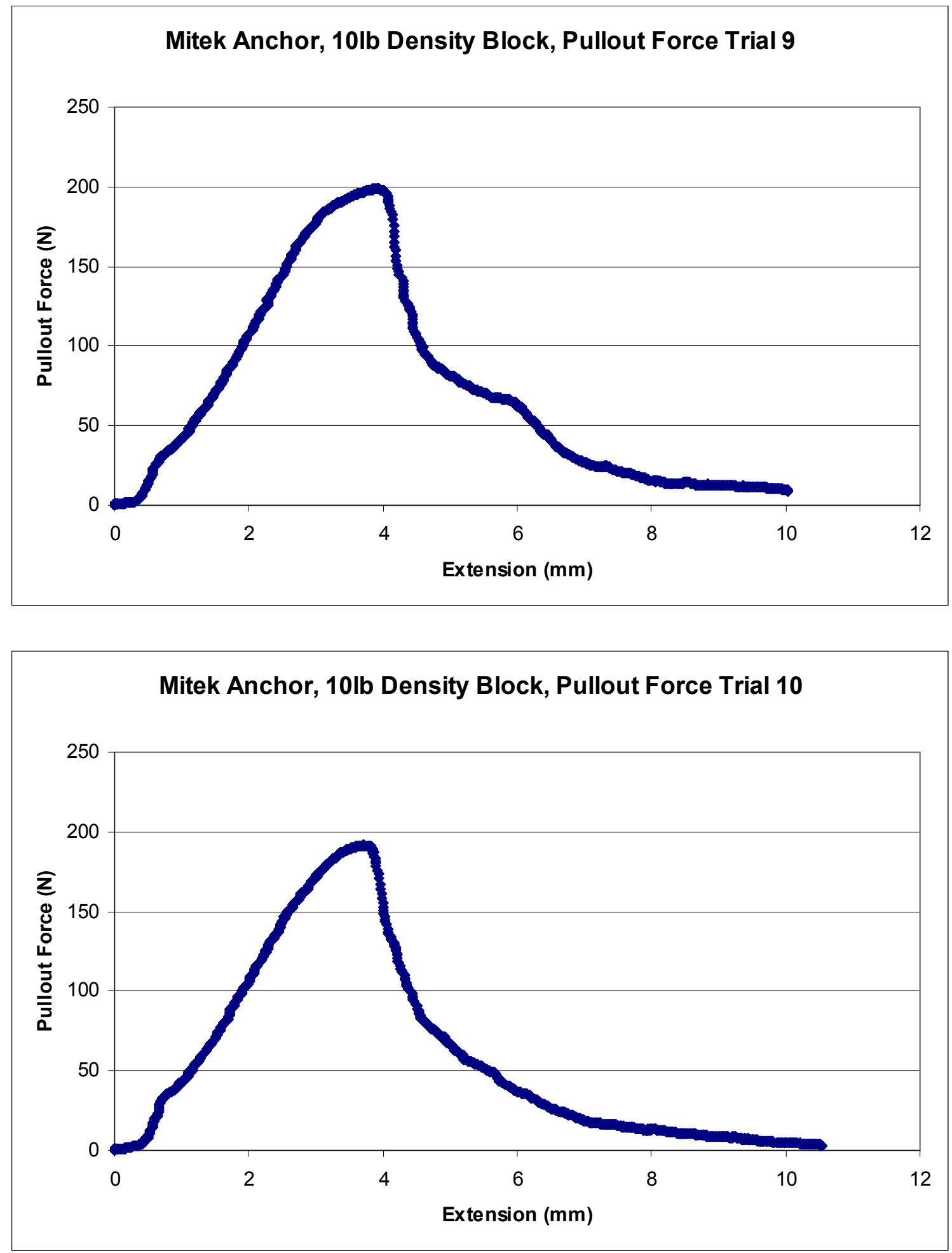

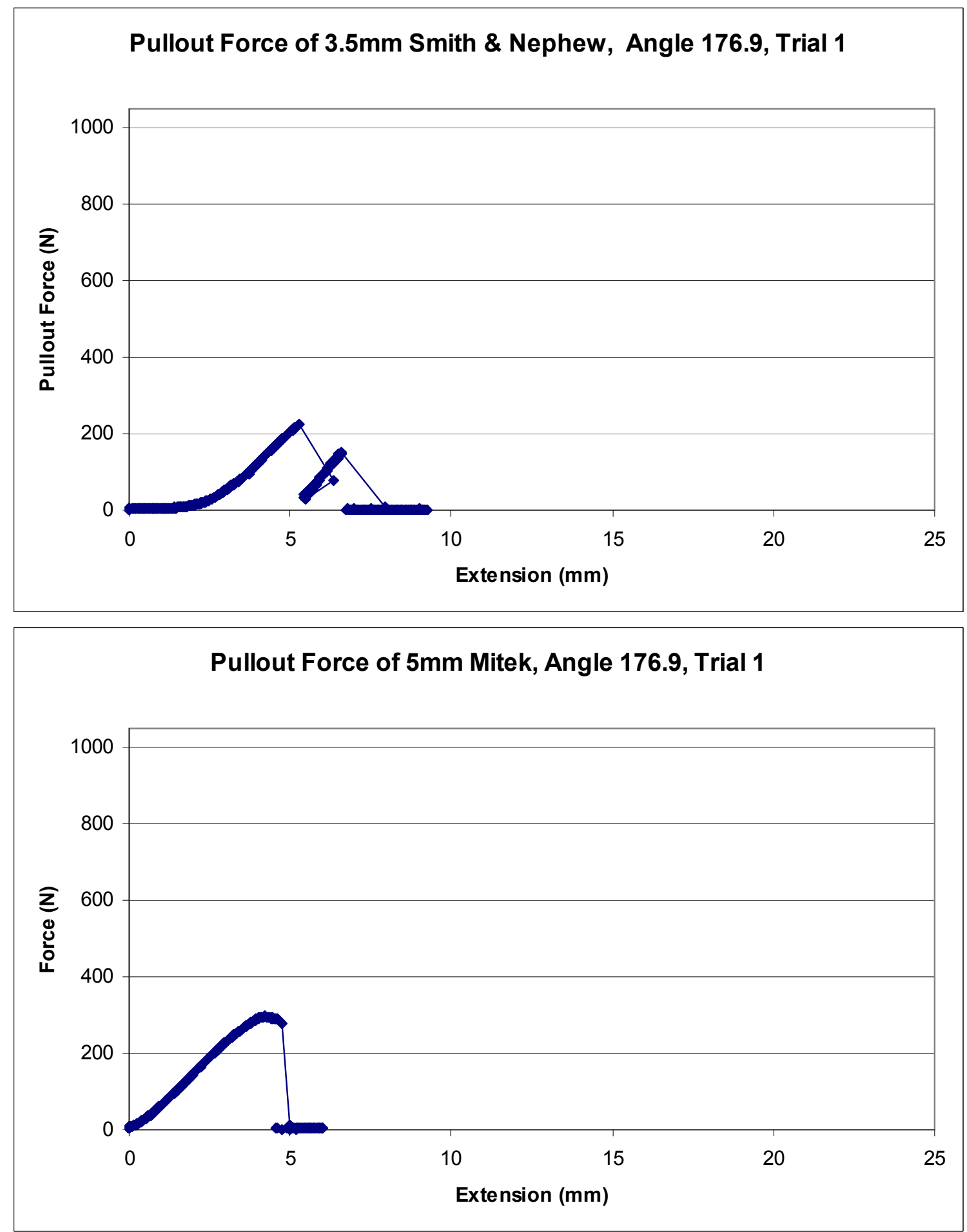

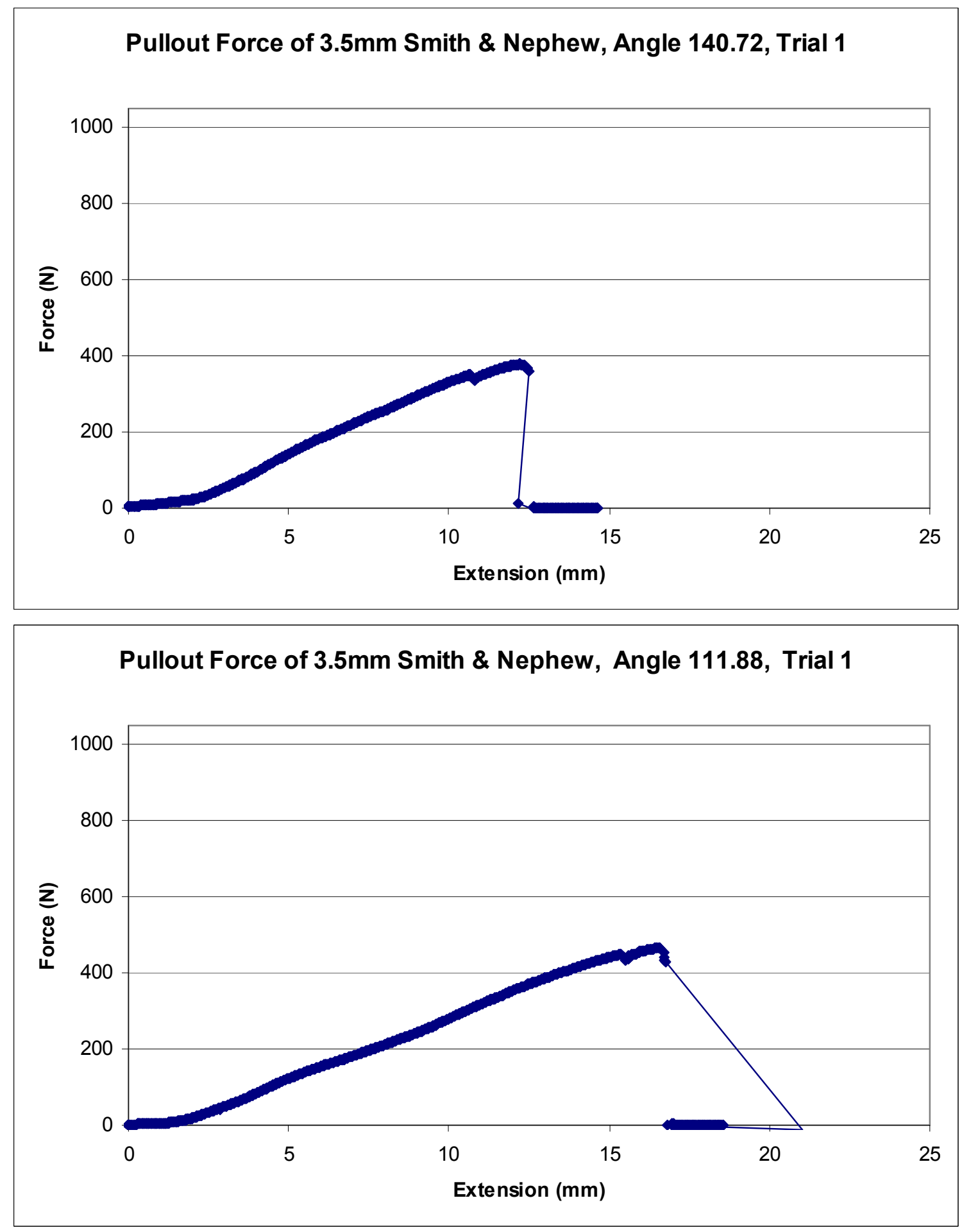

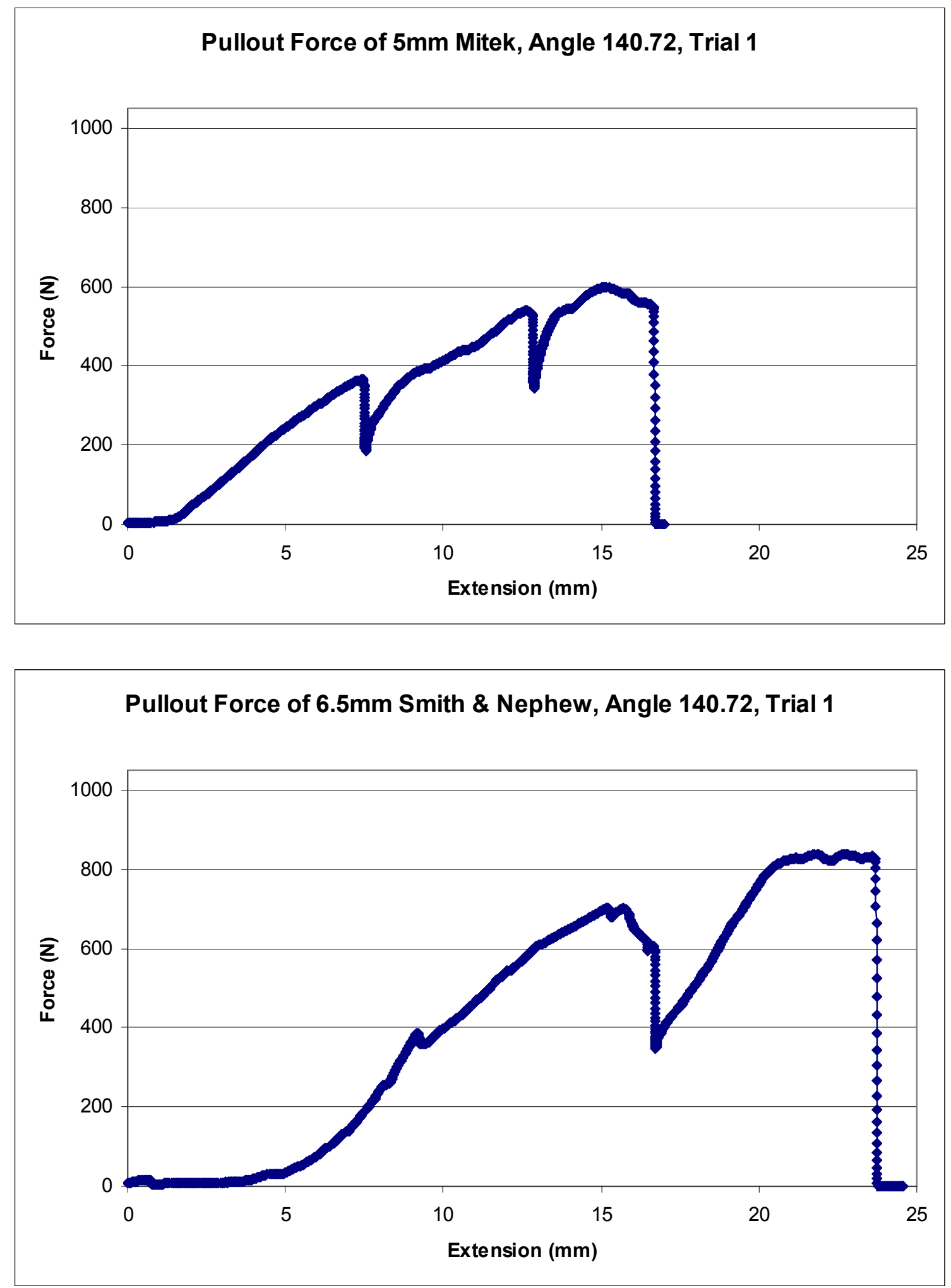

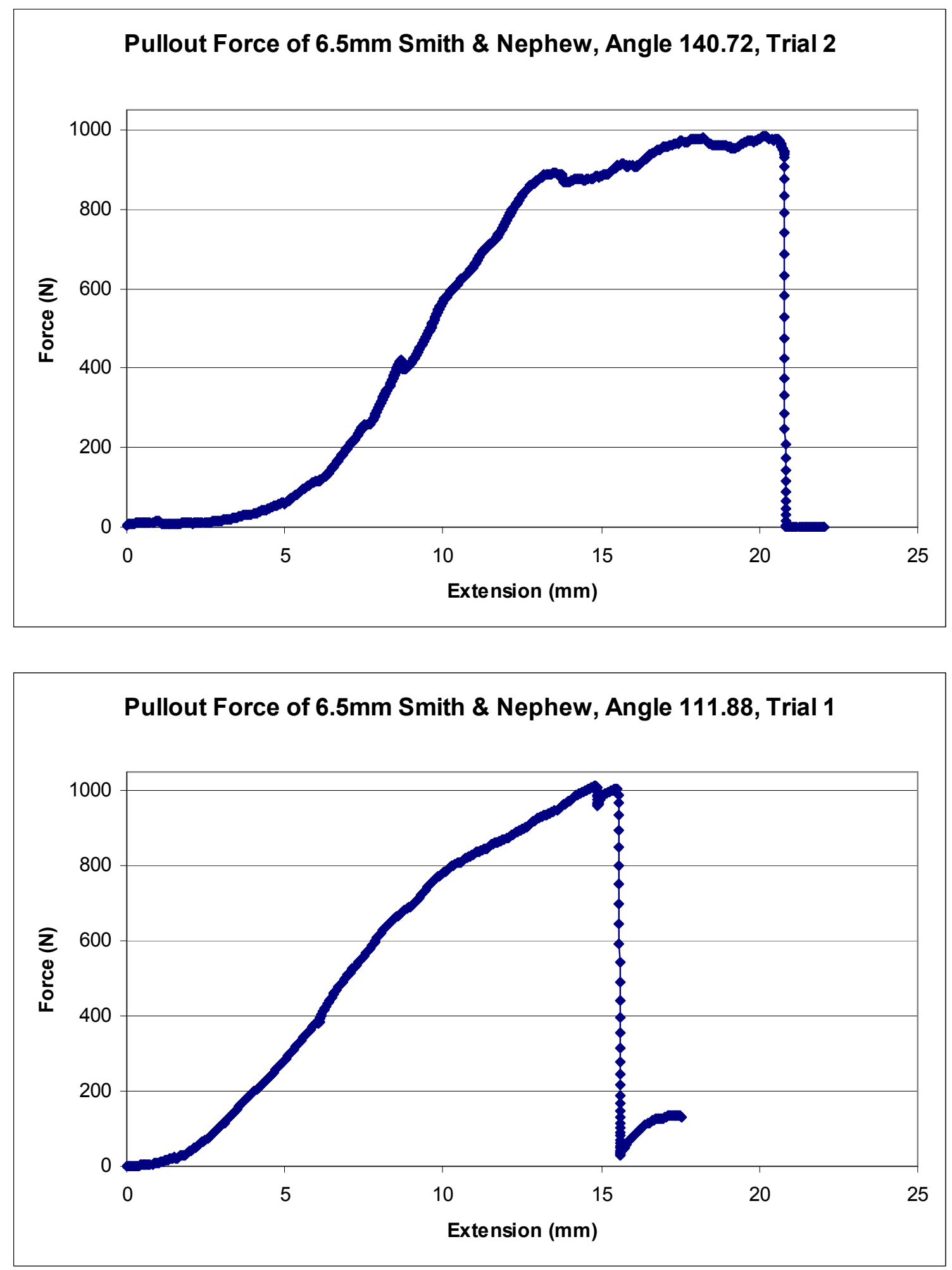

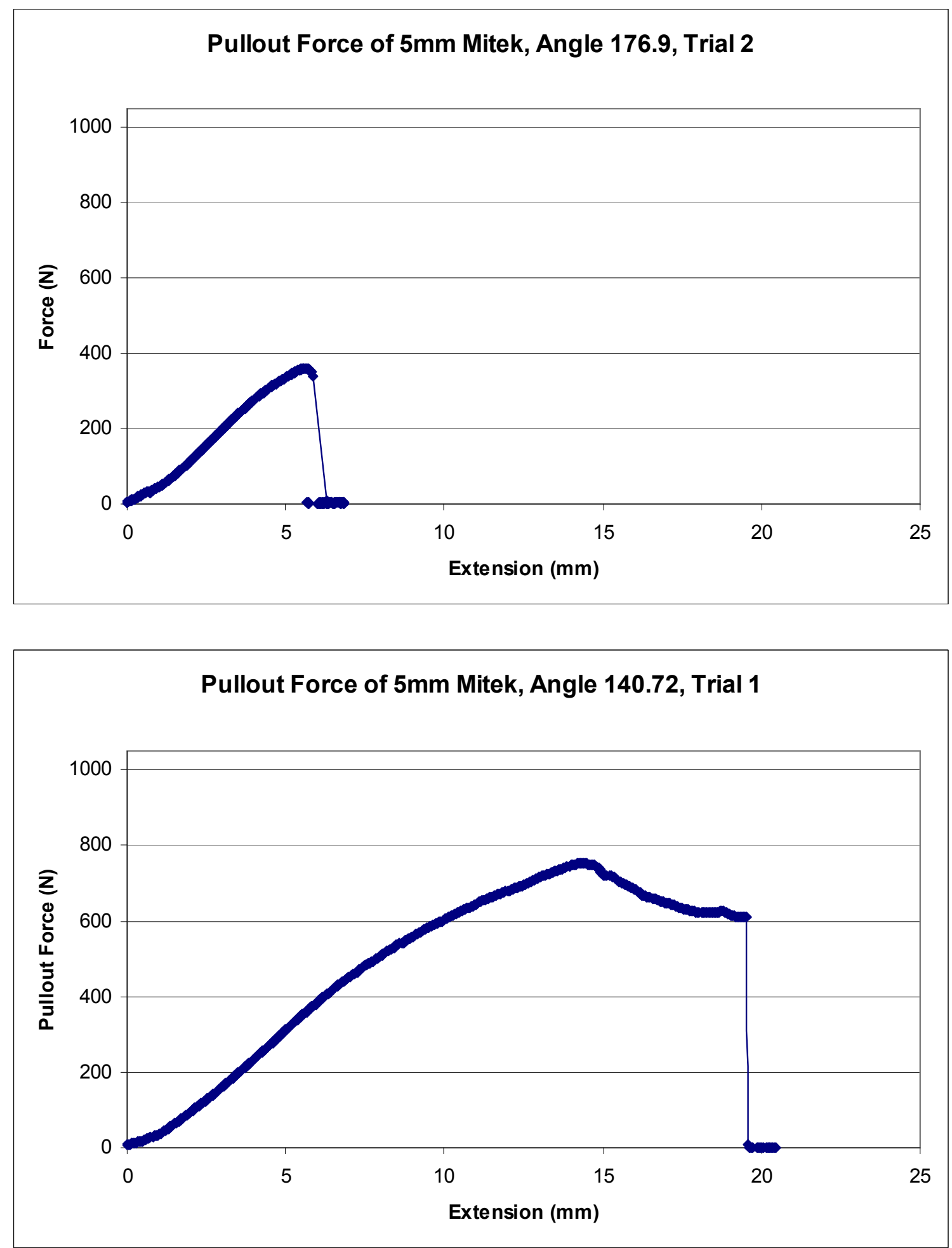

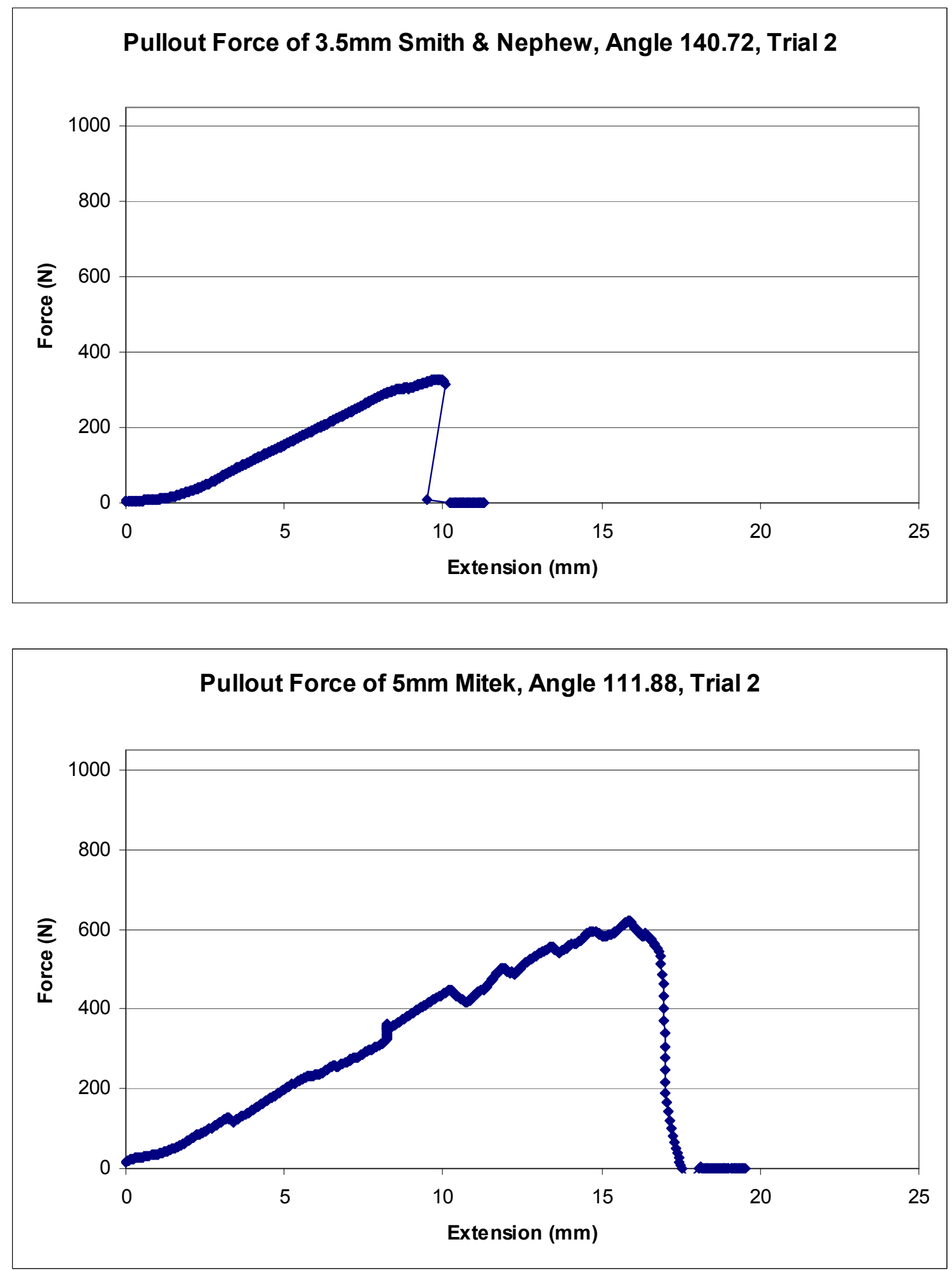

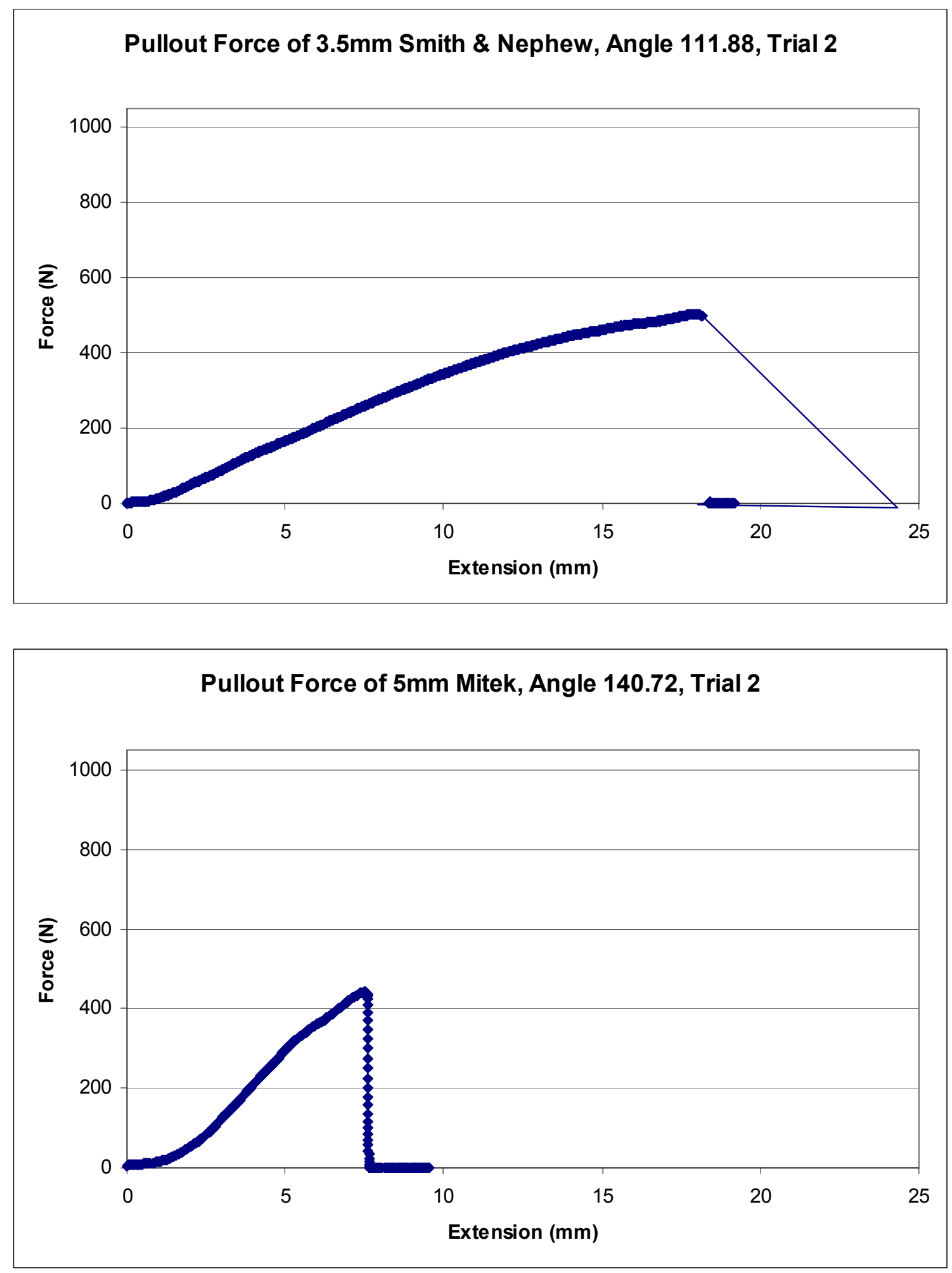

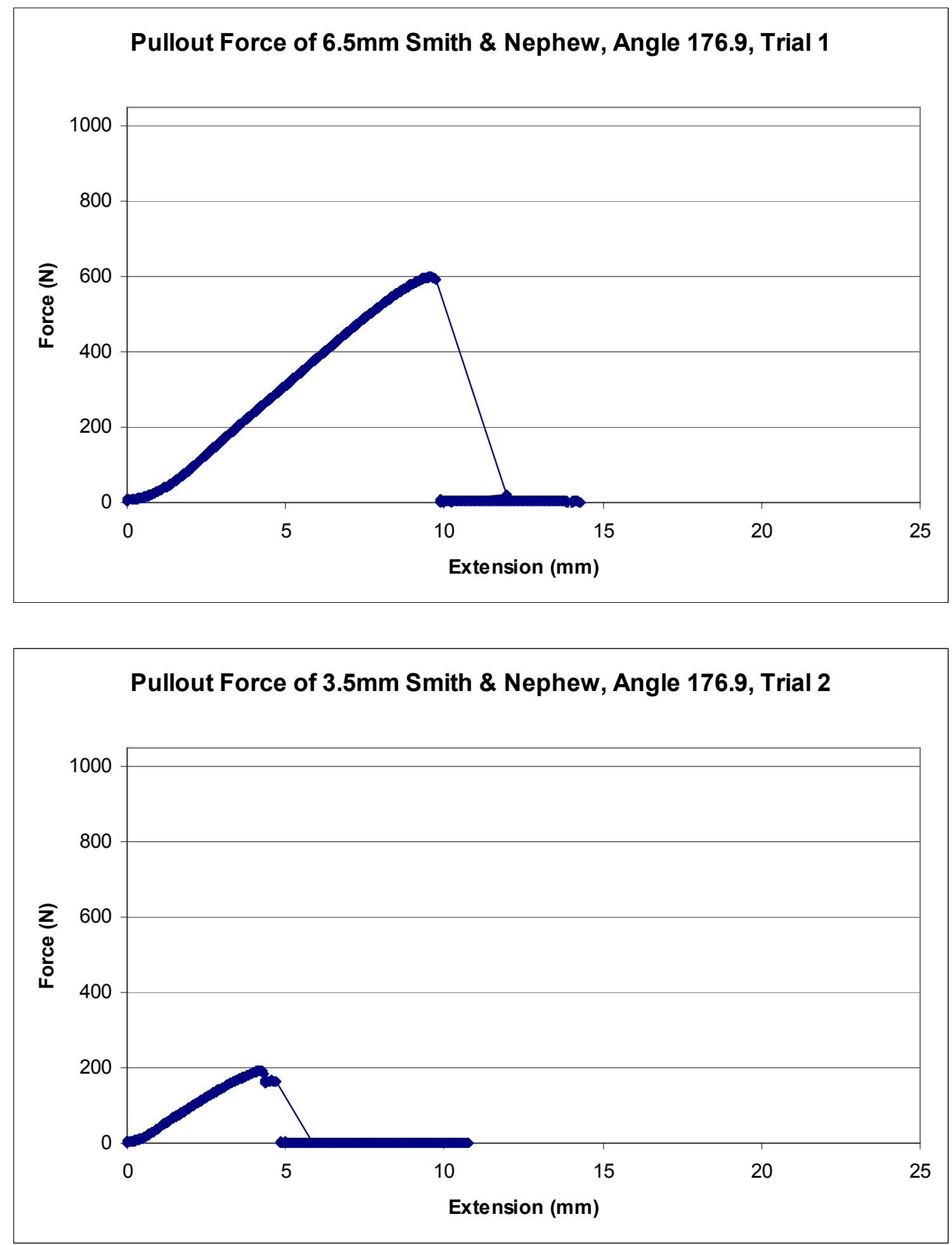

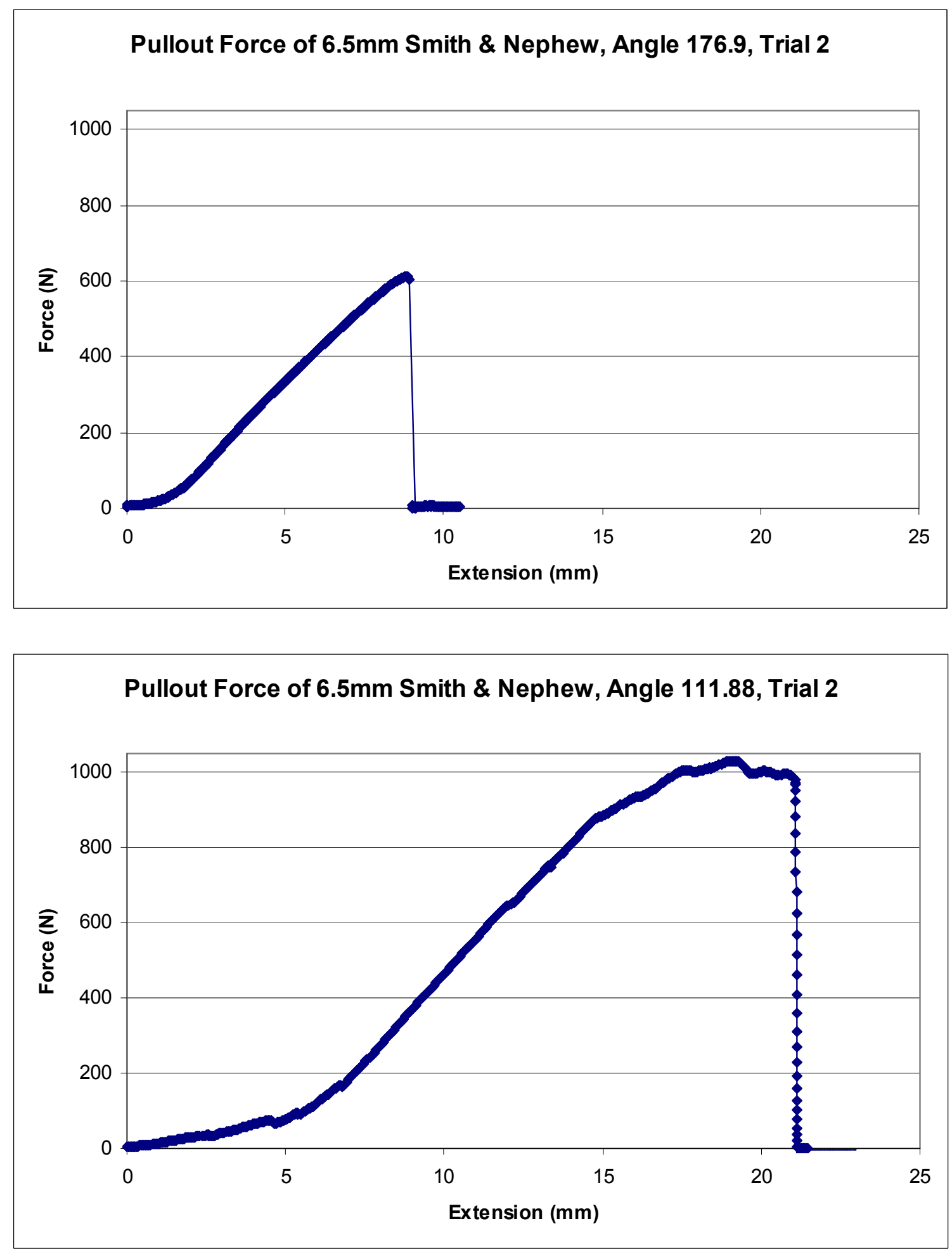
APPENDIX C: Derivation of Parametric Estimate Equations 
Force $=$

$1344.12538699001+(-5.41463211712075 *$ :Angle $)$

+ Match( :Anchor, 1, 277.427777777778， 2, -56.8472222222222，3， $-220.580555555556)$

$+($ :Angle - 143.166666666667) * Match( :Anchor, 1, -1.07078494237471, $2,-0.113769514300098,3,1.18455445667481)$

$\underline{\text { Anchor 1: } 6.5 \mathrm{~mm} \text { Smith \& Nephew }}$

Force $=1344.12-5.41 *$ Angle $+277.43+($ Angle-143.17)*(-1.07)

Force $=-5.41$ Angle -1.07 Angle $+1344.12+277.43+153.12$

$\mathrm{F}=-6.48 *$ Angle +1774.67

Anchor 2: 5mm Mitek

Force $=1344.12-5.41 *$ Angle $-56.85+($ Angle -143.17$) *(-0.114)$

Force $=-5.41$ Angle- 0.114 Angle $+1344.12-56.85+16.321$

Force $=-5.524$ Angle +1303.591

Anchor 3: 3.5mm Smith \& Nephew

Force $=1344.12-5.41 *$ Angle $-220.58+($ Angle -143.17$) *(1.18)$

Force $=-5.41$ Angle +1.18 Angle $+1344.12-220.58-168.94$

Force $=-4.23$ Angle +954.6 
APPENDIX D: Additional JMP Data Analysis 


\begin{tabular}{|c|c|c|c|c|}
\hline \multicolumn{3}{|c|}{ Summary of Fit } & & \\
\hline $\begin{array}{l}\text { RSquare } \\
\text { RSquare } \\
\text { Root Me } \\
\text { Mean of } \\
\text { Observa }\end{array}$ & $\begin{array}{l}\text { quare } \\
\text { conse } \\
\text { (or S }\end{array}$ & $\begin{array}{l}\text { Error } \\
\text { n Wgts) }\end{array}$ & $\begin{array}{r}982763 \\
981327 \\
499205 \\
136.352 \\
40\end{array}$ & \\
\hline \multicolumn{5}{|c|}{ Analysis of Variance } \\
\hline Source & DF & $\begin{array}{l}\text { Sum of } \\
\text { Squares }\end{array}$ & Mean Square & F Ratio \\
\hline $\begin{array}{l}\text { Model } \\
\text { Error } \\
\text { C. Total }\end{array}$ & $\begin{array}{r}3 \\
36 \\
39\end{array}$ & $\begin{array}{r}148270.18 \\
2600.51 \\
150870.70\end{array}$ & $\begin{array}{r}49423.4 \\
72.2\end{array}$ & $\begin{array}{r}684.1887 \\
\text { Prob > F } \\
<.0001\end{array}$ \\
\hline
\end{tabular}

Suture anchor type, polyurethane block density, and interaction of both effects on the pullout force of suture anchors.

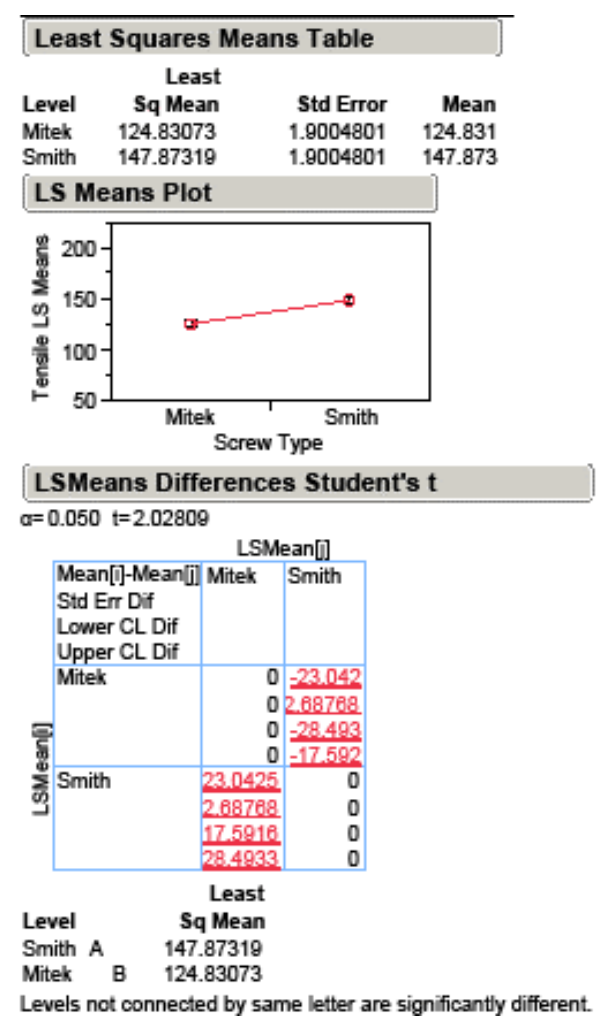

The effects of suture anchor type on pullout force in polyurethane blocks. 


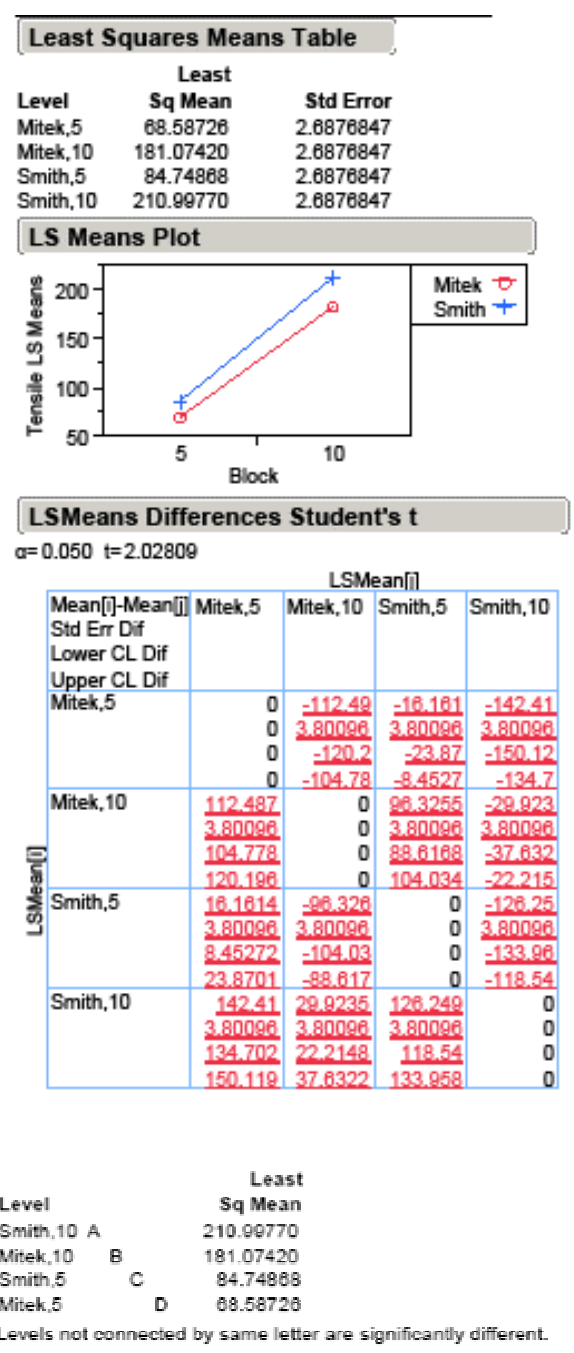

Effect of the interaction of suture anchor and polyurethane block density on pullout force. 